\title{
Confinement of Luminescent Guests in Metal-Organic Frameworks: Understanding Pathways from Synthesis and Multimodal Characterization to Potential Applications of LG@MOF Systems
}

\author{
Mario Gutiérrez, ${ }^{1,2 \dagger}$ Yang Zhang, ${ }^{1 \dagger}$ and Jin-Chong Tan ${ }^{1, *}$

\begin{abstract}
${ }^{1}$ Multifunctional Materials \& Composites (MMC) Laboratory, Department of Engineering Science, University of Oxford, Parks Road, Oxford OX1 3PJ, United Kingdom.

${ }^{2}$ Departamento de Química Física, Facultad de Ciencias Ambientales y Bioquímica, INAMOL, Universidad de Castilla-La Mancha, Avenida Carlos III, S/N, 45071 Toledo, Spain.
\end{abstract}

\footnotetext{
${ }^{\dagger}$ These authors contributed equally to this work.

*E-mail: jin-chong.tan@eng.ox.ac.uk
}

\begin{abstract}
This review gives an authoritative, critical, and accessible overview of an emergent class of fluorescent materials termed "LG@MOF" - engineered from the nanoscale confinement of luminescent guests (LG) in a metal-organic framework (MOF) host, realizing a myriad of unconventional materials with fascinating photophysical and photochemical properties. We begin by summarizing the synthetic methodologies and design guidelines for representative LG@MOF systems, where the major types of fluorescent guest encompass organic dyes, metal ions, metal complexes, metal nanoclusters, quantum dots, and hybrid perovskites. Subsequently, we discuss the methods for characterizing the resultant guest-host structures, guest loading, photophysical properties, and review local-scale techniques recently employed to elucidate guest positions. A special emphasis is paid to the pros \& cons of the various methods in the context of LG@MOF. In the following section, we provide a brief tutorial on the basic guest-host phenomena, focusing on the excited state events and nanoscale confinement effects underpinning the exceptional behavior of LG@MOF systems. The review finally culminates in the most striking applications of LG@MOF materials, particularly the "turn-on" type fluorochromic chemo- and mechano-sensors, non-invasive thermometry and optical $\mathrm{pH}$ sensors, electroluminescence and innovative security devices. This review offers a comprehensive coverage of general interest to the multidisciplinary materials community to stimulate frontier research in the vibrant sector of light-emitting MOF composite systems.
\end{abstract}


Keywords: Luminescent guest (LG); Metal-organic framework (MOF); LG@MOF composite; Nanoconfinement; Guest-host Interaction; Excited state phenomena; Sensors; Electroluminescence; Mechanochromism; Thermochromism; Solvatochromism

Figure for Table of Contents (TOC):

Post-synthetic \& in situ guest confinement

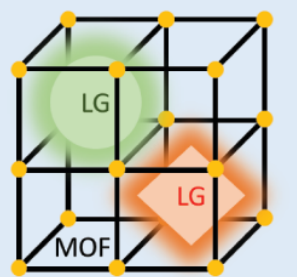

Unique guest-host phenomena
Vast range of

luminescent guests (LG)

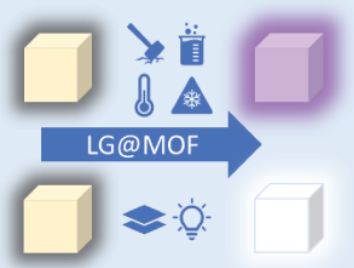

Many potential device applications (sensors lighting, imaging) 


\section{TABLE OF CONTENTS}

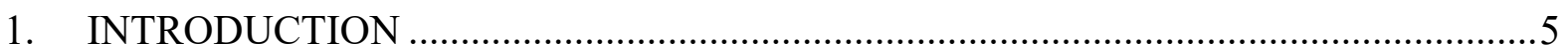

2. SYNTHETIC PROTOCOLS AND DESIGN GUIDELINES .....................................

2.1. Post-synthetic LG confinement methods ...................................................

2.2. In situ $\mathrm{LG}$ confinement methods...................................................................

2.3. Importance of the sample washing step \& the role of guest concentration .............11

3. TYPES OF LUMINESCENT GUEST FOR CONFINEMENT IN MOF .......................12

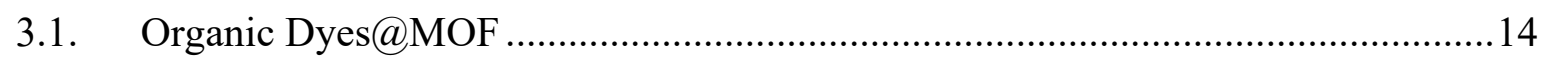

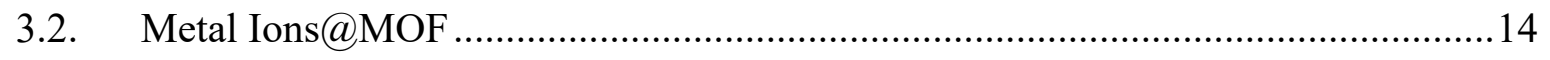

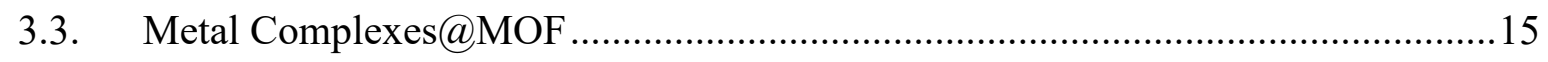

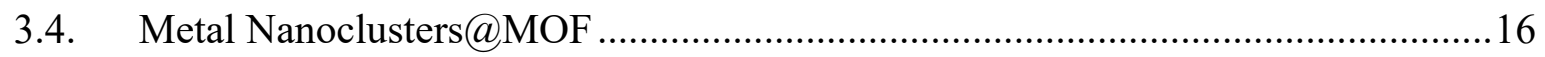

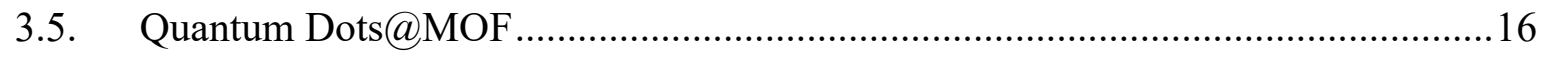

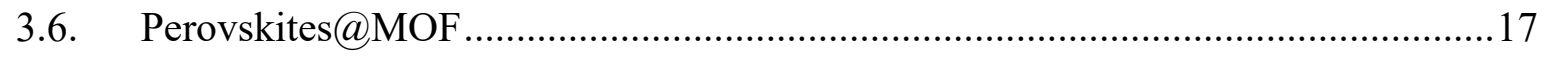

4. CHARACTERIZATION METHODS FOR LG@MOF, UNDERSTANDING THEIR

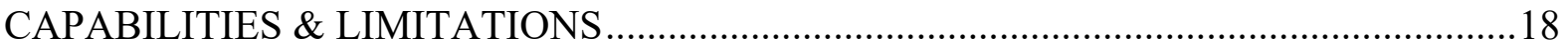

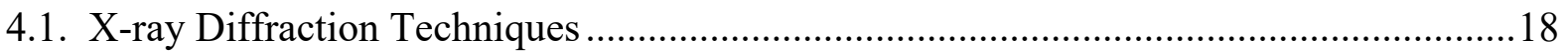

4.2. Steady-State UV-Visible and Fluorescence Spectroscopy …....................................20

4.3. Nuclear Magnetic Resonance (NMR) Spectroscopy …......................................23

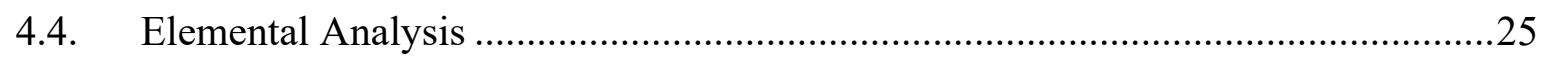

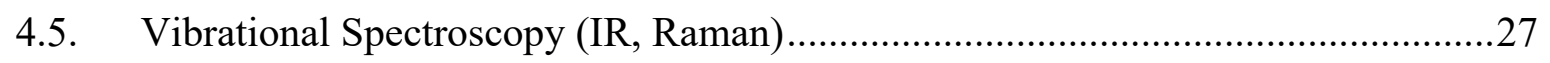

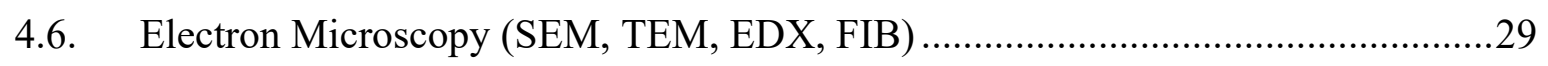

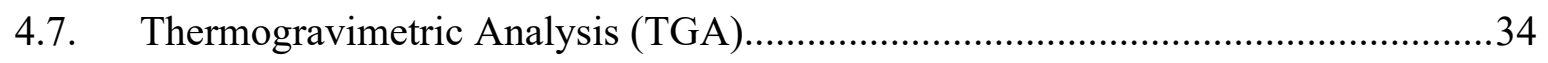

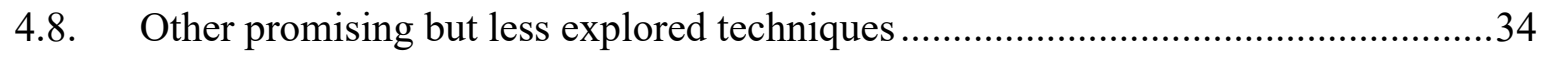

4.8.1 Confocal laser scanning microscopy (CLSM) ................................................

4.8.2 Scattering-type scanning near-field optical microscopy (s-SNOM) coupled with nano-Fourier transformed infrared spectroscopy (nanoFTIR).....................................38

4.8.3 Synchrotron and Neutron Based Techniques............................................ 40 
5.1. Förster Resonance Energy Transfer (FRET)

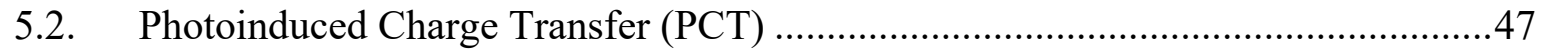

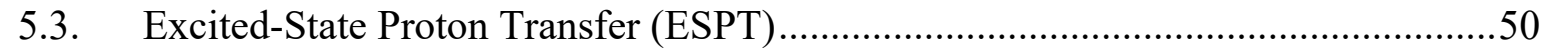

5.4. Confinement Effects, Weak Interactions, and Aggregates Formation ....................52

6. POTENTIAL APPLICATIONS AND DEVICES INTEGRATING LG@MOFs ..........56

6.1 Volatile Organic Compounds, Toxic Chemicals and Gases (VTGs) Detection ......57

6.2 Temperature Sensing and Non-Invasive Thermometry .....................................60

6.3 Mechanochromic Sensors for Force Monitoring and Stress Detection ...................62

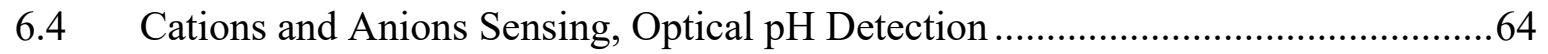

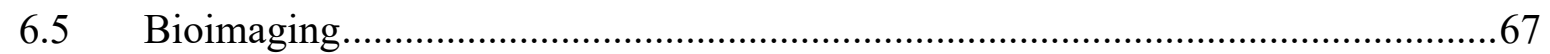

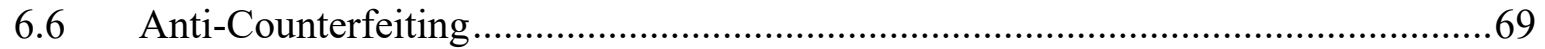

6.7 Light Emitting Devices (LEDs) and Optical Convertors.....................................71

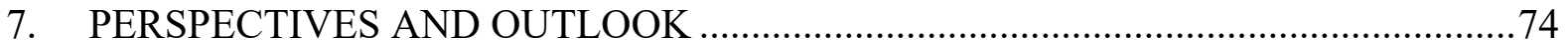

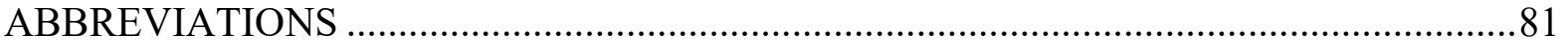

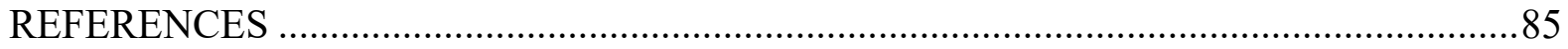




\section{INTRODUCTION}

The pace of progress witnessed in the field of metal-organic frameworks or porous coordination polymers (MOFs or PCPs) is accelerating, transforming it into a central subject of interdisciplinary research worldwide. Whilst it first began in the realm of synthetic chemistry, in the past 25 years $^{1}$ the research on MOF materials has propagated to materials science, engineering, physics, biomedicine, nanotechnology, and other cognate disciplines. MOFs are nanoporous hybrid materials constructed from the self-assembly of organic and inorganic basic building blocks. In essence, MOFs are crystalline compounds with long-range periodic order, whose extended 3-D structures are comprising metal nodes (ions or clusters) connected by multitopic organic linkers. This bottom-up synthetic process generates a cornucopia of network topologies and framework architectures ${ }^{2}$ held together by strong directional bonds, ${ }^{3}$ yielding porous frameworks with a well-defined cavity size (typically $c a$. $0.2-3 \mathrm{~nm}$ ). Starting from a variety of organic and inorganic building units, in principle, one could design, engineer, and tune a plethora of physical and chemical properties which cannot be obtained from purely organic and inorganic systems alone. ${ }^{4}$

Many MOFs are appreciably more porous than commercial nanoporous adsorbents, such as zeolites, silica gels, and activated carbons. ${ }^{5}$ Naturally, the first practical uses identified for MOFs have included gas storage and separation, $\mathrm{CO}_{2}$ capture, and catalysis, which may be perceived as traditional applications for nanoporous materials. However, the potential of MOFs is not just limited to the foregoing applications, and during the last decade there has been a growing interest on the development of a variety of guest-encapsulated MOF systems (Guest@MOF) ${ }^{6}$ and luminescent MOF materials (LMOFs). ${ }^{7,8}$ Indeed, LMOFs and composites have been demonstrated as promising candidates for optoelectronic and photonic applications (e.g., chemical and temperature sensors, solid-state lighting, biomarkers, anti-counterfeiting) hence driving a host of research activities aimed at this direction. ${ }^{9-15}$

One unique way to design and fabricate LMOFs is to harness the porosity of the MOF host to afford the encapsulation of a variety of luminescent guests (LG), resulting in the “LG@MOF” composite system (i.e. a special case of Guest@MOF). Guest encapsulation into MOFs offers certain advantages over traditional syntheses of luminescent materials, such as the facile and cost effectiveness of this methodology, the possibility of tuning the emission properties by a rational selection of off-the-shelf fluorophores or luminescent dyes, and the prevention of aggregation-caused quenching (ACQ) phenomena via partitioning of the 
luminescent guests into the pores of the crystalline MOF host. Although the number of reviews on the subject of luminescent MOFs is growing steadily, the majority of them are focused on LMOF materials with intrinsic luminescence. ${ }^{16-19}$ In this review, therefore, we shall concentrate our efforts on recent exemplars pertaining to the LG@MOF systems, which have substantially pushed the boundaries of LMOFs and offer exciting new opportunities as a platform to engineer bespoke materials for real-world applications.

The review will be organized as follows. Section 2 will introduce the readers to the primary synthetic protocols for the fabrication of LG@MOF materials, where the confinement of luminescent guests through the in situ and post-synthetic pathways will be discussed and contrasted. In Section 3, the topical luminescent guests that have been encapsulated within the MOF hosts are summarized and cogently explained. Section 4 is dedicated to the materials characterization methods most applicable for studying LG@MOFs, in which special attention is paid to elaborating their strengths and limitations, plus opportunities where relevant. The key limitations will be addressed, for instance in confirming pore encapsulation of the guests (rather than surface adhesion), and stressing the need to invoke complementary characterization techniques to confirm success of a chosen guest confinement/encapsulation strategy. In Section 5, we present a concise tutorial on the fundamental guest-host phenomena and excited state events; importantly, our intention here is to give the general reader a better understanding and thus appreciation of the possible mechanisms underscoring the fascinating behavior observed in LG@MOF systems. Finally in Section 6, we highlight some of the most exciting emergent applications arising from the concept of LG@MOFs, including the construction of electroluminescent MOF-LED devices. We shall elucidate how the mechanochromic, solvatochromic, and thermochromic properties of LG@MOFs can be harnessed for sensing physical and chemical stimuli, putting a special emphasis on pioneering optical sensors enabled by the "turn-on" and ratiometric mechanisms.

\section{SYNTHETIC PROTOCOLS AND DESIGN GUIDELINES}

The synthetic methodologies employed have an important impact on the resulting structures and performance of the LG@MOF systems. With regards to the synthesis of pristine MOF materials, the available methods have been fully described and summarized by many comprehensive reviews. ${ }^{20-24}$ In the light of this, for this section we will focus on explaining and discussing the synthetic routes to yield the LG@MOF “composite” systems only. Currently, 
the synthesis of this family of luminescent guest-encapsulated systems can be broadly divided into two major categories. The first approach is termed the "post-synthesis" method as depicted in Figure 1a, while the second strategy illustrated in Figure 1b is designated as the "in situ synthesis" method. In the sections that follow, we shall evaluate the basic concepts and general applicability, discussing the advantages and disadvantages of the fabrication pathways by means of representative examples to enable the readers to develop a better understanding of the processes involved for fabricating LG@MOF materials.

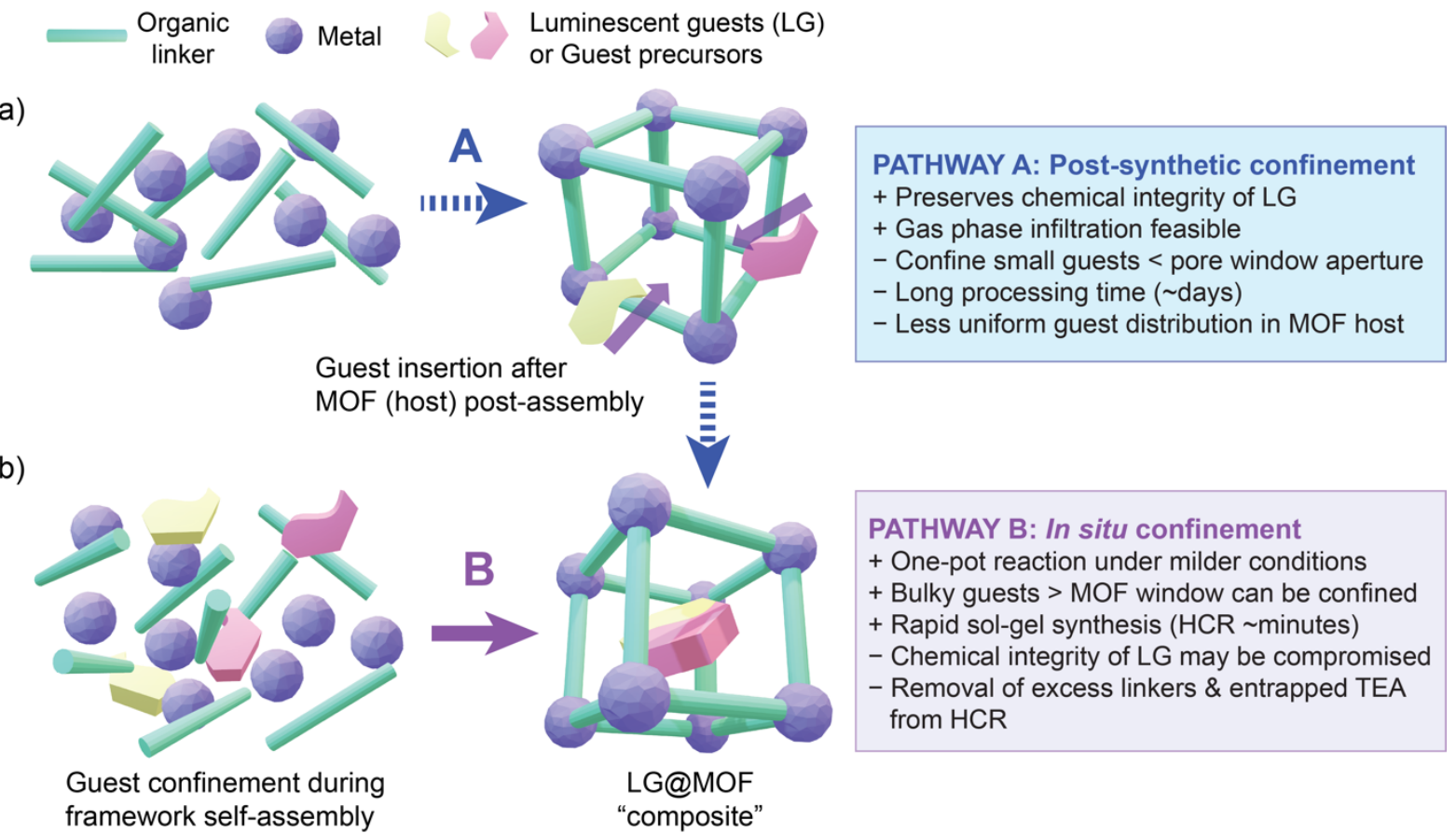

Figure 1. Schematics illustrating the synthetic pathways of the LG@MOF composite system. (a) Post-synthetic confinement, and (b) in situ confinement methodologies to achieve the incarceration of luminescent guests (LG) or guest precursors within the porous MOF host structure. The right panels summarize some of the pros and cons of these guest-host confinement strategies. (TEA $=$ triethylamine, $\mathrm{HCR}=$ high-concentration reaction.)

\subsection{Post-synthetic LG confinement methods}

The post-synthesis methodology begins with the standard synthesis to yield the MOF structure first, followed by sample washing and evacuation or activation steps. Subsequently, the pre-assembled MOF is subjected to a separate process where the insertion of the luminescent guest monomer or precursors into the pores takes place, see pathway A in Figure 1. If it is a monomer, then the size of the guest is required to be relatively smaller than the pore window aperture of the MOF host; if it is a precursor, this will require additional synthesis steps to obtain the desired guest. ${ }^{25,26}$ In general, a significant advantage of the post-synthesis 
method is that it helps to preserve the chemical integrity of the guest species because many luminescent guests may not be stable if introduced directly during MOF synthesis stage. This means that post-synthetic confinement helps to avoid guest exposure to the MOF synthesis environment, where harsh conditions such as acidic or basic media, or high temperature and pressure could degrade the luminescent guest molecules. Nevertheless, the post-synthesis method presents the following shortcomings: time-consuming (up to several days), more complicated synthesis steps, and uneven guest distribution due to differential diffusion rates on the internal and external surfaces of MOF crystals. ${ }^{27}$

At present, the commonly used methods to attain post-synthetic LG@MOF systems are liquid impregnation and gas-phase infiltration. The operation of liquid impregnation is straightforward. In principle, the prepared MOF is immersed in a concentrated solution containing the dye guest molecules intended for encapsulation by the MOF host. Through processes, such as ion exchange and diffusion, the luminescent guest will be absorbed and/or adsorbed by the MOF to form a LG@MOF system. After guest uptake is completed, centrifugation, washing, drying, and other operations are usually performed to remove residual dyes and to collect the resultant LG@MOF crystals. For example, Hu et al. ${ }^{28}$ directly immersed an anionic $\mathrm{Zn} \mathrm{MOF}$ with 1-D channels $\left[\left(\mathrm{CH}_{3}\right)_{2} \mathrm{NH}_{2}\right]^{+}\left[\mathrm{Zn}_{4}\left(\mu_{4}-\mathrm{O}\right)(\mathrm{NTB})_{2}\left(\mathrm{NO}_{2}-\mathrm{bdc}\right)_{0.5}\right] \cdot 3 \mathrm{DMA}$; $\mathrm{NTB}=4,4$ ',4"-nitrilotrisbenzoic acid, $\mathrm{NO}_{2}$-bdc = 2-nitro-4-benzenedicarboxylic acid] into the cationic Rhodamine $\mathrm{B}(\mathrm{RhB})$ in a dimethylacetamide (DMA) solvent for one hour, to yield the RhB@MOF system. Fu et al. ${ }^{29}$ also used this liquid impregnation method, by immersing another $\mathrm{Zn} \mathrm{MOF}\left[\mathrm{Zn}(\mathrm{TIPA})\left(\mathrm{NO}_{3}{ }^{-}\right)_{2}\left(\mathrm{H}_{2} \mathrm{O}\right)\right] \cdot 5 \mathrm{H}_{2} \mathrm{O}$; TIPA = tri(4-imidazolylphenyl)amine] in an aqueous solution of an anionic dye HPTS (8-hydroxy-1,3,6-pyrenetrisulfonicacid trisodium) for two weeks, to attain the dual-emissive HPTS@MOF system.

Compared with liquid impregnation, the operation of gas-phase loading is relatively more complicated as this usually requires specially designed vessels, high temperature, and a low-pressure environment. However, using this method means it is solvent-free, which can avoid the unwanted competition between solvent absorption and guest absorption by the MOF host. For example, Muller et $a .^{30}$ combined the luminescent DXP $[N, N$-bis(2,6dimethylphenyl)-3,4:9,10-perylene tetracarboxylic diimide] guest molecules and a number of different MOFs (MOF-5, MOF-177 and UMCM-1) in a specially designed glass tube under inert gas conditions (Ar). Subsequently, evacuation, sealing, and heating operations $\left(300^{\circ} \mathrm{C}\right)$ were applied and retained under static conditions for several days to yield the DXP@MOF materials. Tu et al. ${ }^{31}$ demonstrated the post-synthetic sublimation approach to encapsulate 
anthracene (ANT) in gas phase into the pores of ZIF-8. The pre-activated ZIF-8 powder and ANT were placed together in a Schlenk tube and evacuated $(0.1 \mathrm{mbar})$, then heated at $120^{\circ} \mathrm{C}$ for 3 days. The resultant ANT@ZIF-8 system shows a promising reversible yellow-to-purple photo-switching of its fluorescence.

\subsection{In situ $\mathrm{LG}$ confinement methods}

LG@MOF systems can also be fabricated by employing the in situ nanoconfinement method. This encapsulation approach can simply be described as the guest molecules being combined directly with the MOF's basic building blocks (i.e., the metal source and organic linkers) in the same vessel prior to formation of MOF structure, see pathway B in Figure 1. Facilitated by host-guest interactions during coordination directed assembly, bulky guest molecules, ${ }^{32}$ especially those with a size larger than the MOF window aperture, could be successfully encapsulated within the MOF pores, or immobilized in the MOF crystal structure to give a "core-shell" type composite. Compared with the post-synthesis confinement method (Section 2.1), the in situ approach is relatively simpler with shorter reaction times, and could yield a better result in terms of guest distribution. However, one important factor that must be considered is the stability of the guest species during in situ encapsulation process, so that their chemical integrity is not compromised under the synthesis conditions of the MOF host; to this end, typically milder synthetic conditions are used.
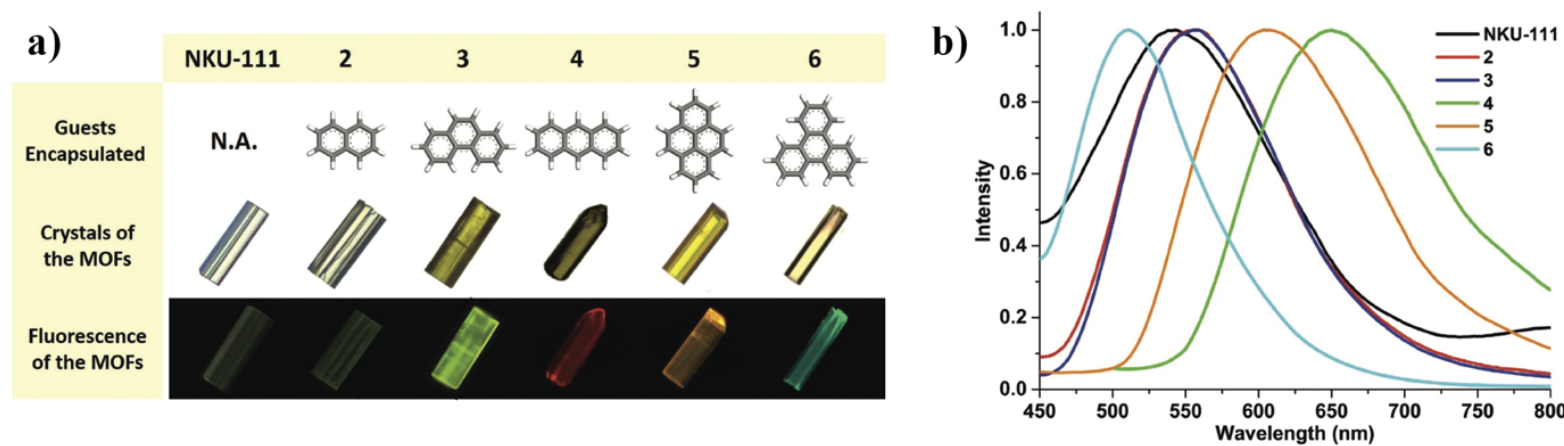

Figure 2. (a) Luminescent guest@NKU111 series showing the guest-dependent optical and luminescent images of the crystals, where the confined fluorophores comprise naphthalene (2), phenanthrene (3), anthracene (4), pyrene (5), and 9,10-benzophenanthrene (6). (b) Normalized emission spectra of the LG@NKU111 series determined under an excitation wavelength of $420 \mathrm{~nm}$. Adapted with permission from ref. 33. Copyright 2018 John Wiley \& Sons.

The simplest and most common way to achieve in situ synthesis of LG@MOF is via onepot reaction. The process directly combines in a reaction mixture the dye guest molecules, metal salts, and organic linkers of the intended MOF, and then allows the coordination directed 
self-assembly to take its own course to completion. For example, Zhang et al. ${ }^{33}$ used the onepot method to successfully prepare a series of LG@NKU-111 system with tunable luminescent properties, see Figure 2. It was reported that the host-guest assembly (heated for 1 day at $100{ }^{\circ} \mathrm{C}$ ) was facilitated by the donor-acceptor interaction forming between the electron-rich fluorescent guests and the electron-deficient host framework. Asadi et al. ${ }^{34}$ also demonstrated a one-pot method performed under room temperature for the encapsulation of polyethylene glycol-capped ZnS quantum dots (PEG-ZnS QD) within the ZIF-67 MOF. To this end, a solution containing the organic linker (2-methylimidazole) and the PEG-ZnS QD, were mixed with another solution containing the metal salt $\left(\mathrm{Co}\left(\mathrm{NO}_{3}\right)_{2} \cdot 6 \mathrm{H}_{2} \mathrm{O}\right)$ and the reaction was stirred for 45 min, yielding the luminescent hybrid material PEG-ZnS QD@ZIF-67.

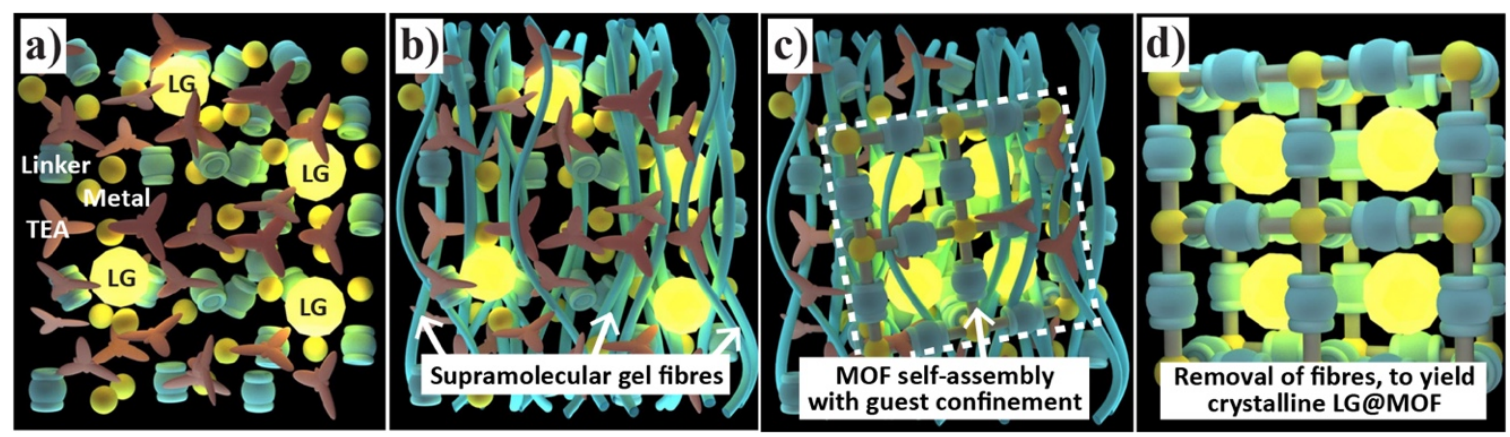

Figure 3. Schematic illustrating the sequential stages of the in situ encapsulation process by HCR method to yield LG@MOF. (a) High-concentration reactants are combined with luminescent guests (LG presented as yellow sphere). (b) Formation of a supramolecular gel fiber network facilitating the self-assembly of (c) MOF host confining the luminescent guest molecules. (d) LG@MOF crystals are harvested by disintegrating the scaffolding fibers in a solvent. Adapted from ref. 35. Copyright 2017 John Wiley \& Sons.

More recently, an in situ guest encapsulation strategy termed the "high-concentration reaction" (HCR) method has been developed for synthesizing a variety of LG@MOF systems (Figure 3). ${ }^{35,36}$ Briefly, the synthesis by HCR involves the following key steps: (A) first, prepare a metal salt solution containing the metal centers of the intended MOF host; (B) second, prepare another solution of organic linkers combined with fluorescent guests dispersed in a suitable solvent; (C) finally, solutions A+B are combined to initiate the reaction, where a gellike material coexisting with LG@MOF crystals forms immediately (Figure 4a-b). Resultant LG@MOF crystals can be isolated from the supramolecular gel by washing in an organic solvent. Notably, HCR method produces a faster reaction rate and higher sample yield than the conventional one-pot approach described above. This is generally caused by the use of deprotonation agents, such as triethylamine (TEA), which is typically added to the linker 
solution to accelerate the deprotonation rate of ligands. For instance, the ZnQ@OX-1 ${ }^{35}[\mathrm{ZnQ}$ $=\operatorname{zinc}(\mathrm{II})$ bis(8-hydroxyquinoline) $], \quad \mathrm{Gaq}_{3} @ Z \mathrm{ZIF}-8^{37} \quad\left[\mathrm{Gaq}_{3}=\right.$ gallium(III) tris-(8hydroxyquinoline)], RhB@ZIF-7136 $\left[\mathrm{RhB}=\right.$ rhodamine B] and perylene@ZIF-8 ${ }^{38}$ with fluorochromic sensing properties (Sections 6.1 to 6.4) have been prepared by leveraging the HCR methodology. Because of the effect of downsizing, typically the crystals obtained from this method will be relatively small, giving 2-D morphologies such as nanoplates and nanosheets (Figure 4c). Whilst the characterization of the nanosized crystals is more challenging as they require high-resolution SEM, TEM or AFM techniques (Section 4), they do offer better processability and improved photoluminescent properties such as a high quantum yield ( $\Phi>90 \%$ ) via electrospun nanofibers. ${ }^{36}$
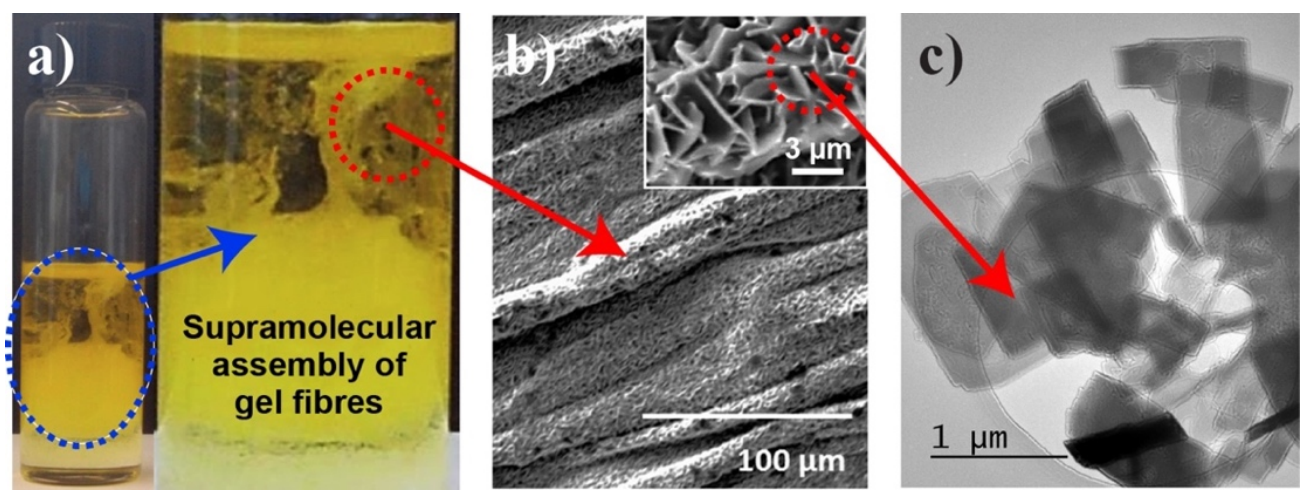

Figure 4. (a) In situ synthesis of a LG@MOF material employing the HCR method, where the fluorescent guest is $\mathrm{ZnQ}$, resulting in the formation of (b) supramolecular gel fibers coexisting with nanosheets of ZnQ@OX-1 (SEM images and inset). (c) TEM micrograph of the nanosheets obtained through downsizing of the 3D framework of the OX1 MOF. Adapted from ref. 35. Copyright 2017 John Wiley \& Sons.

\subsection{Importance of the sample washing step \& the role of guest concentration}

No matter which method is used, it is critical that the obtained LG@MOF samples are washed thoroughly in an appropriate solvent to remove residual guests adhered to the MOF surface. Because during the synthesis process, some guest molecules will inevitably be adsorbed on the outer surface of the MOF crystals, where these external guests are not protected by the MOF host. Properties of any unprotected fluorophores will be susceptible to degradation by the external environment, ${ }^{39,40}$ which may have an adverse effect on the luminescent properties and photostability of the LG@MOF system as a whole. In general, the obtained LG@MOF material should be washed multiple times (with repeated sonication and centrifuging steps) until the mother liquor becomes clear and non-emissive. For example, with 
sufficient washing cycles, the $\mathrm{ZnQ} @ \mathrm{OX}-1$ material ${ }^{35}$ can exhibit interesting sensing properties for acetone vapor, but it will only emit a constant blue light without any sensing behavior if being washed once or twice. However, too much washing could risk removal of weaklyconfined guests from the pore, leading to a deterioration of fluorescent properties, especially when employing channel-type MOFs as a host. ${ }^{28,41}$ Hitherto, the cases of sample failure or poor batch-to-batch sample reproducibility that may be linked to either too little or too excessive washing of resultant guest-encapsulated samples are rarely reported in the literature; this synthetic issue clearly deserves more attention to help the community improve the overall reproducibility of reported LG@MOF systems.

Another critical aspect which will have a direct impact on the luminescent properties of the LG@MOF material is the guest concentration within the MOF host. Two key factors must be considered when synthesizing a LG@MOF system, in order to maximize the luminescence quantum yield of the material and to possibly tune the color emission. On the one hand, if the concentration of the guest is too high, most probably the dyes will aggregate, and as a consequence, the fluorescence quantum yield (QY) will generally decrease by ACQ (although in some cases QY may increase because the dyes experience aggregation-induced emission, AIE), and then the emission will generally be red-shifted due to the formation of J-aggregates (see Section 5.4). On the other hand, if the concentration is too low, the dyes will be incorporated mainly as isolated monomers within the pores of the MOF. However, even if the luminescence quantum yield of the composite material may be high (as luminescence quantum yield is a ratio between the emitted and absorbed photons), the total fluorescence intensity of the material could be very low, as the amount of fluorescent guest present is relatively low per unit mass (see Section 4.3). Hence, it is vital to be able to precisely adjust the concentration of the LG within the MOF, in a way that the concentration is high enough to yield a strong luminescent material, but not excessively concentrated to prevent the formation of a large population of unwanted aggregates.

\section{TYPES OF LUMINESCENT GUEST FOR CONFINEMENT IN MOF}




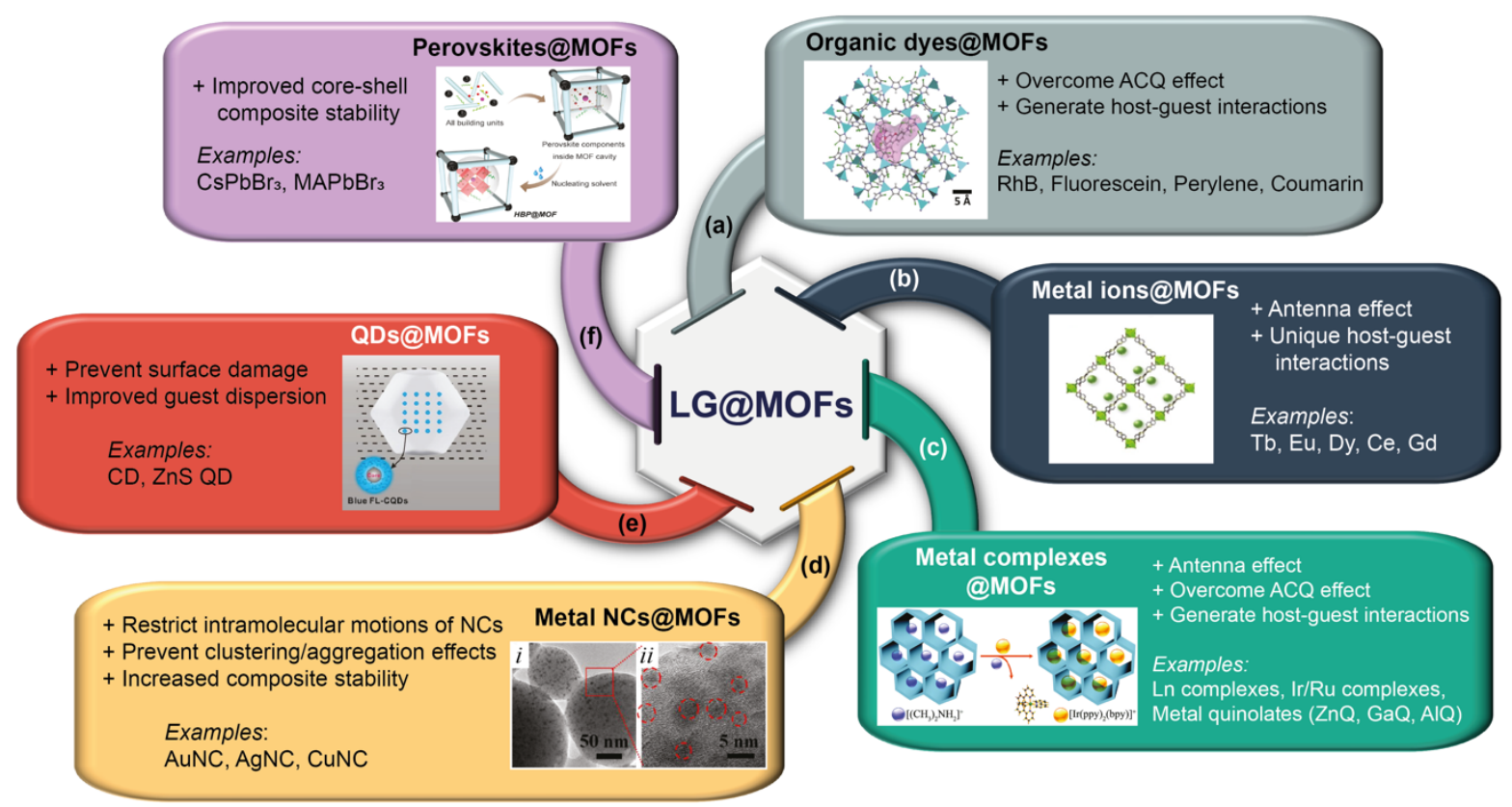

Figure 5. Major types of luminescent guest (LG) that have been encapsulated inside the MOF host to yield the LG@MOF composite systems with specific advantages summarized. (a) organic dyes, figure inset adapted from ref. 43. Copyright 2020 American Chemical Society. (b) metal ions, figure inset adapted with permission from ref. 52. Copyright 2019 Elsevier. (c) metal complexes, figure inset adapted from ref. 54. Copyright 2017 Royal Society of Chemistry. (d) metal nanoclusters (NCs), figure inset adapted from ref. 63. Copyright 2018 American Chemical Society. (e) quantum dots (QDs), figure inset adapted from ref. 68. Copyright 2014 American Chemical Society. and (f) hybrid perovskites, figure inset adapted from ref. 26. Copyright 2019 American Chemical Society. Panels (d)-(e) are representative examples of core-shell composites.

Due to the high tunability of MOF structures, it is unsurprising that the types of luminescent guests are also diverse. In general, for most types, MOFs can help to improve and enhance the luminescent performance of the encapsulated guests, mainly including: (1) Reducing ACQ effect and allowing many guests that can only fluoresce in solution to achieve luminescence in the solid-state. (2) Imposing a caging effect on the guests, thereby reducing the non-radiative decay of the guests and increasing their lifetime and quantum yield $(\Phi)$. (3) There are many MOFs with good thermal stability and water stability, such as ZIF-8, UiO-66, and ZIF-71. Using these stable MOF hosts as a "shield" can protect and improve the stability of the confined guests and broaden their scope of application (Section 6). At present, the main types of luminescent guest commonly studied in the context of LG@MOF are summarized in Figure 5, which encompass the organic dyes, metal ions/complexes/nanoclusters, quantum dots, and inorganic-organic (hybrid) perovskites. The following sections will present some topical examples of luminescent guests being confined in MOFs. 


\subsection{Organic Dyes@MOF}

Organic luminescent dyes are a widely used luminescent material, the luminescence of which stems from the $\pi-\pi$ conjugation of their chromophoric groups. This kind of material has a relatively broad emission spectrum, may have a high quantum yield, and some of which have luminescence tunability, but most face the limitation of ACQ effect and can only emit in solution. Importantly, the organic dyes@MOF systems can effectively overcome the ACQ effect and achieve a solid-state emission. For example, the RhB@bio-MOF-1 reported by Chen et $a l .{ }^{42}$ has helped the confined RhB guests to overcome the ACQ effect and thus yield luminescence in the solid state.

Apart from addressing the ACQ effect, the organic dye@MOF systems may also enhance or widen the luminescence tunability of the composite, which will significantly advance its sensing, display, and light emission applications (Section 6). For instance, the extensively studied RhB@MOF systems broaden the solvatochromism of RhB itself by allowing the RhB@ZIF-71 system to achieve different luminescent response even in nonpolar solvents (e.g., toluene, hexane, cyclohexane), whereas the pure $\mathrm{RhB}$ molecule has poor solubility or is insoluble, hence, precluding its luminescence by ACQ. ${ }^{43}$ Moreover, the encapsulation of the organic dye guests in the MOF pores can give rise to novel mechanochromic materials due to changes in the inter-chromophoric distances with an applied pressure, which may enhance, quench, or shift the emission behavior under stress. Exemplars of mechanochromic LG@MOF systems are presented in Section 6.3. For a comprehensive list of commonly used smallmolecule fluorescent dyes (e.g., fluoresceins, rhodamines, coumarins, naphthalimides, cyanines, pyrene), with descriptions of their structures and photophysical properties, the reader may consult a number of specific reviews dedicated to this subject matter. ${ }^{44,45}$

\subsection{Metal Ions@MOF}

The metal ions used in the LG@MOF systems are mainly lanthanide (Ln) ions. ${ }^{46}$ The Ln ions display sharp characteristic emission peaks, they are generally stable in the ambient environment and their luminescence is governed by the forbidden $\mathrm{f}-\mathrm{f}$ transitions. ${ }^{47}$ Using the periodic structure of MOF to confine Ln ions means this will enable a larger number of photons to be emitted per unit volume. ${ }^{48}$ Moreover, the organic linker of MOF is highly tunable, which facilities MOF interaction with the Ln ions, promoting energy transfer or establish the antenna effect for enhancing the luminescence efficiency of Ln ions. Together, these factors may help 
to raise the quantum yield of the confined systems to about $14-30 \%{ }^{49-51}$ Since the energy transfer between the MOF and Ln ions is sensitive to the external environmental stimuli, the metal ions@MOF system can attain an optical sensing response. For example, the Tb@ZnMOF system reported by Ji et al. ${ }^{52}$ exhibits a noticeable energy transfer/antenna effect between the linker and the terbium (Tb) ions. This kind of host-guest interaction was exploited to prepare a turn-on type sensor that responds to aspartic acid, see further exemplars in Section 6.1.

\subsection{Metal Complexes@MOF}

Metal complexes themselves are also diverse, but different metal complexes face different challenges to reach practical applications. Confinement of metal complexes in a MOF host can further tailor their luminescent properties, expand applications, and even give several metal complexes new sensing capabilities.

Rare-earth Ln complexes are extensively studied in the context of luminescent MOFs. ${ }^{11,12,53}$ The advantages and disadvantages of the Ln complex are akin to those faced by the Ln ions (Section 3.2), employing MOF for the confinement of Ln complex may help to overcome some outstanding problems. However, most of the rare earth elements are toxic, costly and scarce. Other metal complexes with good luminescent performance have become more attractive, such as Iridium (Ir) and ruthenium $(\mathrm{Ru})$ based complexes. Ir and Ru complexes possess high luminescence efficiency and tunable emission, but they are also affected by the ACQ effect to a certain extent. Some researchers have successfully applied Ir and Ru complexes to construct the metal complex@MOF systems, which suppress the ACQ effect and produce a white light emission where the quantum yield was found to be $\Phi \sim 15-20 \% .{ }^{54,55}$

Another promising family of electroluminescent non-rare-earth metal complexes being investigated for confinement in the LG@MOF system is the metal quinolate series, denoted as $\mathrm{Mq}_{\mathrm{n}}$ (e.g., $\mathrm{Alq}_{3}, \mathrm{Gaq}_{3}, \mathrm{Inq}_{3}, \mathrm{Scq}_{3}, \mathrm{Znq}_{2} ; \mathrm{q}=8$-hydroxyquinoline). ${ }^{56}$ The metal quinolates have excellent luminescent performance, good stability, and relatively low cost. The construction of Mqn@MOF systems can make full use of the advantages offered by this kind of complexes, as demonstrated by a recent example where Gaq $@$ @ZIF-8 is incorporated in an electroluminescent device (LED) as an electroactive layer (further details in Section 6.7). ${ }^{37}$ Interestingly, the hostguest interaction between the $\mathrm{Mq}_{\mathrm{n}}$ and $\mathrm{MOF}$ can generate unique sensing properties. For example, ZnQ@OX-1 system incorporating a zinc(II) bis(8-hydroxyquinoline) complex (ZnQ or $\mathrm{Znq}_{2}$ ) shows a fast and reversible fluorescence sensing response towards acetone vapor. ${ }^{35}$ 


\subsection{Metal Nanoclusters@MOF}

Luminescent metal nanoclusters (NCs: e.g., gold, silver, copper) are composed of several to tens of metal atoms, translating to a nominal size of $c a \cdot 1-2 \mathrm{~nm} \cdot{ }^{57}$ Because their size is comparable to the Fermi wavelength of electrons, metal NCs receive a strong quantum confinement effect, resulting in unique luminescent properties. In general, they have a relatively broad emission, and because of their non-toxicity, metal NCs are attractive for use in the biomedical field (Section 6.5). Nevertheless, compared with other luminescent materials, such as organic dyes, the quantum yield of metal NCs is low, ${ }^{58-61}$ which dramatically limits their further development.

Some studies ${ }^{59,60}$ have suggested that restricting intramolecular motions of metal NCs can improve the quantum yield. That can be realized by confining metal NCs within the MOF scaffold to yield a core-shell composite. Importantly, the immobilization of NCs in the MOF host prevents clustering and aggregation effects that will degrade luminescent performance. ${ }^{62}$ For example, Gao et al. ${ }^{63}$ used the system of AuNCs@ZIF-8 to boost the quantum yield of Au NCs by approximately 4.5 times, where $\Phi$ was reported to rise from $7.6 \%$ to $33.6 \%$ after their immobilization in the ZIF-8 host. Furthermore, MOF crystals can also play a protective role for some unstable metal $\mathrm{NCs}$, such as $\mathrm{Cu},{ }^{64}$ which is susceptible to oxidation (Section 4.6). It has been reported that when confined within the scaffolding of the MOF host, the stability of the $\mathrm{Cu}$ nanoclusters can be substantially prolonged from 3 days to 3 months. Given its promise, there is an increasing number of research focusing on the development of metal NCs@MOF systems. On this note, while silver NCs@zeolite systems show many intriguing luminescent properties, ${ }^{62}$ there is still very limited work exploring the use of MOFs to afford nanoconfinement of silver NCs. ${ }^{65}$

\subsection{Quantum Dots@MOF}

Quantum dots (QDs) are defined as semiconductor nanocrystals with a diameter of about 2-10 nm. Like the metal nanoclusters mentioned above, QDs are also subject to a strong quantum confinement effect due to their small size, leading to a good optical performance, including a broad excitation range, high quantum yield, high extinction coefficients, and tunable luminescence. ${ }^{66}$ Generally, although some materials are not semiconductors, they are often discussed as QDs, especially carbon dots (CDs), also known as carbon quantum dots. In addition to having excellent luminescent properties, CDs also possess chemical inertness and 
better biocompatibility, which can be deployed in biomedicine. However, QDs face some challenges in terms of their application. One limitation is that the luminescent properties of QDs, particularly the quantum yield, is greatly affected by surface defects. Thus, some additional protection is needed to prevent surface damage. Another drawback is that it is difficult to achieve a good dispersion of QDs during use, which may negatively impact their luminescence.

MOFs are proposed to be an effective solution to these problems faced by QDs. For example, Wang et al. ${ }^{67}$ developed a CD@ZIF-8 luminescent system and show that the use of MOF solves the problem of $\mathrm{CD}$ quenching by preventing the formation of aggregates. Furthermore, such QDs@MOF systems can also bestow novel sensing properties. For instance, BPEI-CDs [branched poly-(ethylenimine)-capped carbon quantum dots]@ZIF-8 system reported by Lin et al. ${ }^{68}$ exhibits $\mathrm{Cu}^{2+}$ sensing properties, in which the role of the nanoporous MOF host was also to help accumulate the target analytes, thereby enhancing its detection sensitivity towards $\mathrm{Cu}^{2+}$. Further examples will be discussed in Section 6.4 focusing on cations and anions sensing applications.

\subsection{Perovskites@MOF}

Perovskites have attracted more and more attention because of their low cost, high quantum yield, and tunable optical properties. But this class of framework material has a well-known problem - poor stability. Different mechanisms, ascribed to a range of intrinsic factors (e.g., chemical structure, defects, phase and thermodynamic stability) and extrinsic factors (e.g., moisture, thermomechanical, light, oxidation induced) could cause the degradation of hybrid perovskites and impact their long-term performance. ${ }^{69}$

Some relatively stable MOFs, such as the ZIF and UiO series, have been employed in combination with perovskites in an attempt to develop stable perovskite@MOF systems with improved chemical stability and photoluminescence. For example, Zhang et al. ${ }^{70}$ successfully improved the stability of halide perovskites through encapsulation in UiO-67, resulting in a core-shell type composite. The luminescent properties of the prepared $\mathrm{CsPbBr}_{3} @ \mathrm{UiO}-67$ was reported to remain unchanged for 30 days under ambient atmospheric conditions. Likewise, Mollick et al. ${ }^{26}$ have reported a series of MAPbBr 3 @ZIF-8 with tunable photoluminescent properties, where the protection offered by MOF host has increased chemical stability of the resultant LG@MOF composites. In terms of the potential technological applications of 
perovskite@MOF systems, the reader may refer to Section 6.2 on temperature sensing and Section 6.6 on luminescent anti-counterfeiting inks.

Section 3 gives an overview of the different types of luminescent guests (LG) suitable for confinement within a MOF host, where each category clearly has its advantages and constraints. The representation examples of the luminescent guests summarized in this section will be further elucidated in the context of their applications as LG@MOF systems in Section 6. Next, in Section 4, we will expose the readers to state-of-the-art characterization tools for probing guest inclusion within MOFs, with the intention to demonstrate how these tools are best employed in a complementary fashion while emphasizing the pros and cons of each technique.

\section{CHARACTERIZATION METHODS FOR LG@MOF, UNDERSTANDING THEIR CAPABILITIES \& LIMITATIONS}

Regardless of the methodology followed for the fabrication of the LG@MOF composites, it is very plausible that the guests can be adsorbed onto the surface instead of or in addition to encapsulated into the pores or entrapped in the crystals (core-shell type). While different techniques provide information about the structure, chemical composition, and physicochemical properties; proving the encapsulation of the guest in the MOF host is certainly not a trivial task and this usually requires the use of complimentary techniques. In this section, we will discuss and critically assess the advantages and limitations of some of the most employed techniques for the characterization of LG@MOF systems.

\subsection{X-ray Diffraction Techniques}

Powder X-ray diffraction (PXRD) is a rapid and non-destructive technique used for the structural characterization of powders and microcrystalline materials. For this reason, it has become a routine technique for the determination of the crystalline structure of MOFs and especially of LG@MOF composites, where the inclusion of guests can induce a distortion in the long-range periodicity of the MOF crystals. Generally, the crystalline structure of MOFs remains unaltered after the encapsulation of the photoactive compounds, ${ }^{32,37,71-83}$ mainly because a series of factors such as the MOF rigidity, the guest size, the low loading percentage, 
or the adsorption of the guest onto the MOF surface instead of being encapsulated. However, there are a few interesting examples in which guest encapsulation produces structural modifications in the network. ${ }^{35,84}$ For instance, the flexible $[\mathrm{Zn}(\mathrm{ndc})(\mathrm{o}-\mathrm{phen})] \cdot \mathrm{DMF}_{\mathrm{n}} \mathrm{MOF}$ exhibits notable changes in its PXRD pattern upon desolvation of DMF solvent molecules. ${ }^{84}$ The changes in the MOF structure were determined through in situ PXRD measurements coupled with $\mathrm{CO}_{2}$ adsorption, where it was demonstrated that an increase in the cell volume with $\mathrm{CO}_{2}$ uptake (from $2143 \AA$ to $2963 \AA$ ), reflecting an expansion of the framework. This observation could be extrapolated to the inclusion of four different aromatic amine compounds, which gave rise to PXRD patterns quite distinct from the one of the pristine material alone, indicating a structural reorganization after guest encapsulation. ${ }^{84}$ Similar phenomenon was observed for the Zn-based OX-1 (( $\left.\left.\mathrm{H}_{2} \mathrm{NEt}_{2}\right)_{2}\left[\mathrm{Zn}_{3} \mathrm{bdc}_{4}\right] \cdot 3 \mathrm{DEF}\right) \mathrm{MOF}$, whose Bragg diffraction peaks were shifted after the encapsulation of the luminescent $\mathrm{ZnQ}$ metal complex, corresponding to an expansion of the unit cell parameters (Figure 6). ${ }^{35}$

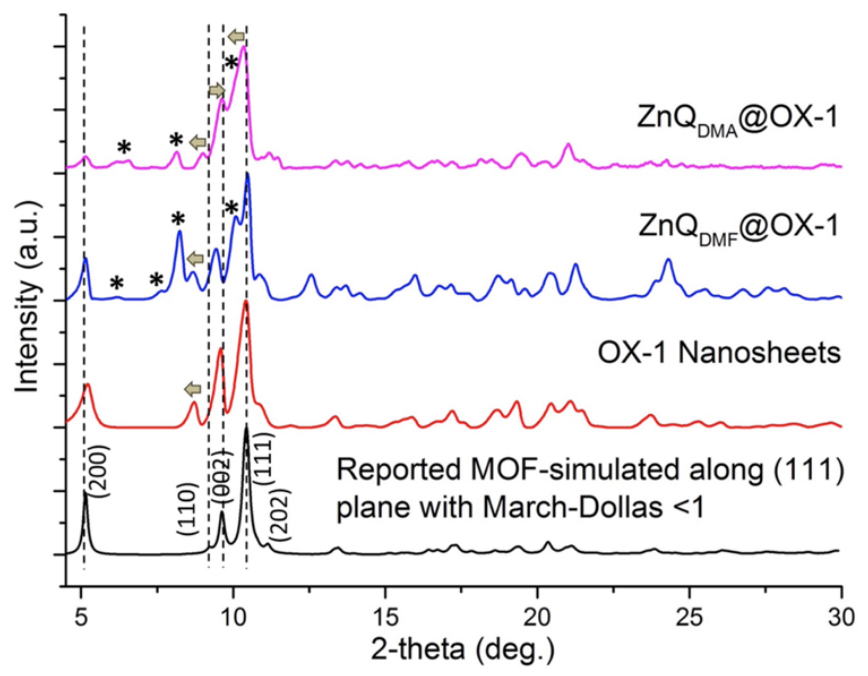

Figure 6. PXRD pattern of the OX-1 nanosheets, with and without confinement of the ZnQ guest molecules, compared with the simulated diffraction pattern of the pristine OX-1 MOF. Asterisks mark the peak positions of the ZnQ luminescent guest. Adapted from ref. 35. Copyright 2017 John Wiley \& Sons.

Although PXRD is a convenient tool for structural characterization, it is not the most suitable technique for the detection of confined guests. There is usually a lack of signal associated with the active guests owing to two main reasons: (i) to detect Bragg diffraction peaks, the guests should have long-range periodicity, however, they are more likely to be randomly distributed within the MOF host; and (ii) the weight \% of confined guests in 
comparison to the MOF host is typically low, ${ }^{85-88}$ and it is well-known that PXRD is not a very sensitive technique (typical detection limit is of the order of 5-10 wt.\%). ${ }^{89}$ On the contrary, single-crystal (SC) XRD enables a more precise identification of the presence and position of confined guests in the MOF structure. For example, the encapsulation of the ionic form of the luminescent solvent green $7\left(\mathrm{SG}^{3-}\right)$ as a counterion in the large channels of FIR-53 $\left.\left(\mathrm{Zn}_{2}(\mathrm{TIPA})_{2}\left(\mathrm{OH}^{-}\right)\right]\left(\mathrm{NO}_{3}{ }^{-}\right)(\mathrm{SG} 7)_{2 / 3} \cdot 5 \mathrm{H}_{2} \mathrm{O}\right) \mathrm{MOF}$ was confirmed by SCXRD (Figure 7), being the first example of the inclusion of a $\pi$-conjugated organic small molecule via a single-crystal to single-crystal transformation. ${ }^{90}$ However, SCXRD presents an inherent limitation related to the sample preparation, which has to be a single crystal of a considerable size and as defectfree as possible. On the one hand, this is of course, not a minor task, and on the other hand, it will hinder the applicability of those materials, whose synthesis must be easily scaled up for practical implementations (Section 6).

a)

a)

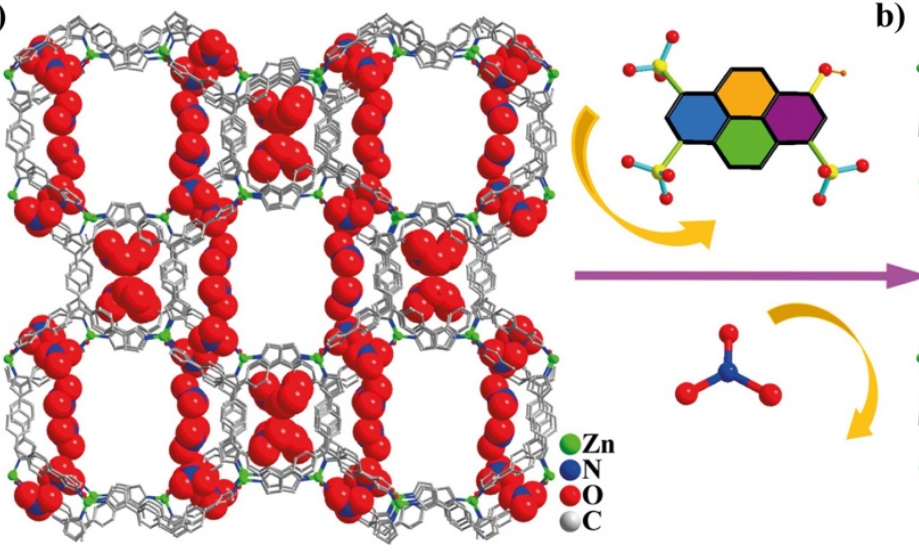

b)

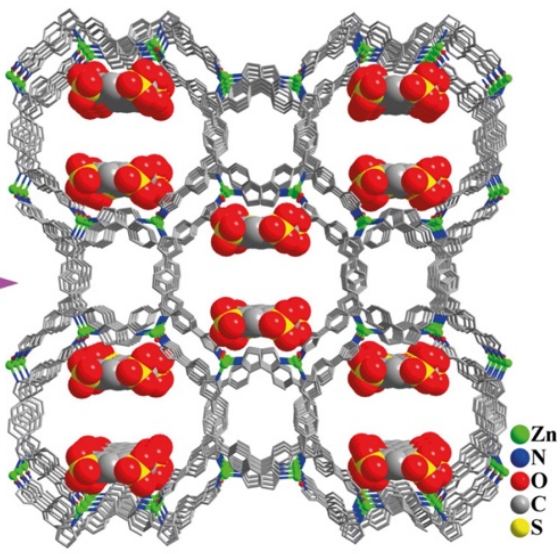

Figure 7. Representation of the crystalline structure of (a) the 3D framework of FIR-53 MOF, and (b) the structure of the MOF upon the encapsulation of the luminescent guest (SG7) into the channels, to yield SG7@FIR-53. Adapted with permission from ref. 90. Copyright 2018 American Chemical Society.

\subsection{Steady-State UV-Visible and Fluorescence Spectroscopy}

Steady-state UV-visible absorption/reflectance and fluorescence spectroscopic techniques are essential for characterizing the optical properties of the ground and electronically excited states of LG@MOF systems. They will also allow the quantification of the fluorescent guests interacting with the MOF hosts. For instance, the loading percentage of guests interacting with a specific MOF can be estimated by measuring the optical density at the maximum intensity of the guest solution before and after its interaction with the MOF. Furthermore, it is also possible 
to calculate the mass of the loaded dye by knowing the molar extinction coefficient of the guest, which can be estimated through UV-visible absorption by applying the Beer-Lambert formula. ${ }^{91}$ The percentage of guest loading can be calculated through the following equation:

$$
\% \text { Guest loading }=\frac{O \cdot D \cdot \text { dye solution }-O \cdot D \cdot \text { supernatant }}{O \cdot D \cdot \text { dye solution }} \times 100
$$

where O.D. stands for the optical density, the dye solution corresponds to the initial solution before its interaction with the MOF, and the supernatant is the solution after being loaded in the MOF. For a reliable characterization of the \% guest loading, the key facts below must be considered: (i) obviously, the O.D. of the supernatant must be always lower than that of the initial dye solution, as a certain amount of dyes will be interacting with the MOF. (ii) A key factor is that the volume of the dye solution and the supernatant must be exactly the same as the $O . D$. is directly proportional to the concentration. If, for example, the solvent of any of these solutions is evaporated during the process, the O.D. values will be higher because the concentration of this solution will also rise. (iii) Finally, as described in the Section 2.3. , it is important to wash the sample properly and one may consider that in the second or even in the third supernatant the amount of dye might not be negligible. In those cases, it is important to determine the O.D. of these solutions and add the values to the total O.D. of the supernatant. If all these points are carefully met, UV-visible absorption spectroscopy, an affordable, rapid and routine technique becomes a powerful instrument to estimate the amount of not only luminescent but many different types of guest entrapped in different host materials. ${ }^{92}$ Indeed, many reports have assessed the loading percentage of fluorescent guests in MOFs by employing this methodology. ${ }^{73,85,93,94}$

UV-visible absorption and fluorescence spectroscopies are also routinely employed for the qualitative characterization of the fluorescent guests in MOFs. As the fluorescent guests usually exhibit characteristic absorption and emission spectra, the detection of those signals in the LG@MOF system is frequently used as a proof for the presence of the luminescent guest. $35,54,75,78,85,88$ For instance, the confinement of $\mathrm{Gaq}_{3}$ in the pores of ZIF-8 MOF was confirmed by the absorption, excitation and emission spectra, corresponding to the characteristic bands of the fluorescent guest (Figure 8a-b).${ }^{37}$ Additionally, increasing the amounts of loaded guest will induce an increase in the absorption intensity, and generally accompanied by a rise in the emission intensity of the LG@MOF composite as well (however, it can sometimes produce a decrease of the emission intensity owing to undesired physical effects, such as inner filtering), 
and these changes in the signal intensity have been correlated with the amount of fluorescent guest in the MOF host (Figure 8c). ${ }^{35,54,75,78,85,88}$
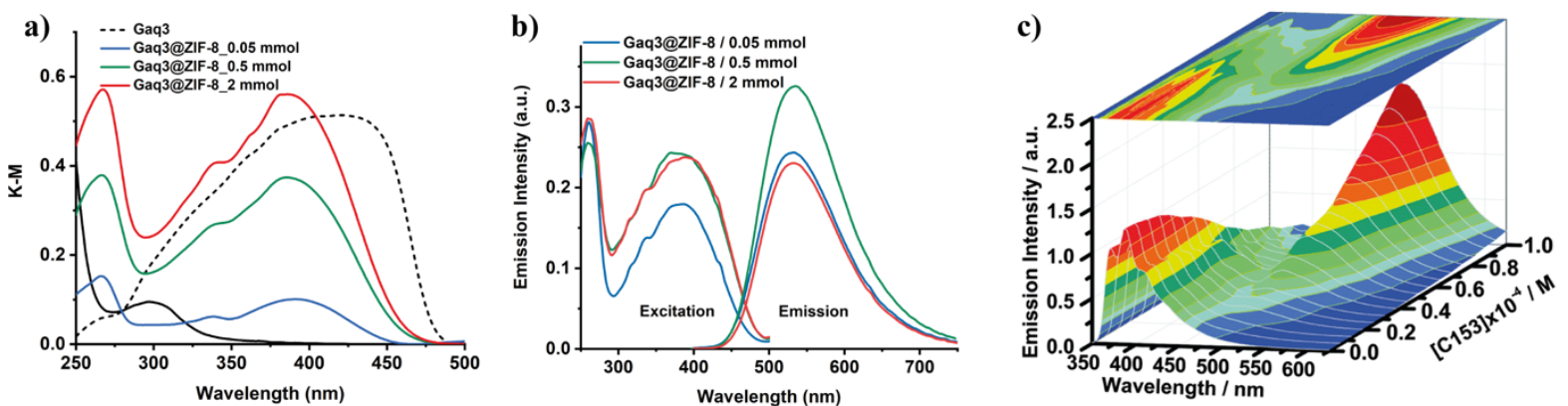

Figure 8. (a) Diffuse reflectance spectra transformed to Kubelka-Munk (K-M) functions, (b) excitation and emission spectra of three samples of Gaq 3 @ZIF-8 with an increasing amount of guest confined in the ZIF8 host. Adapted from ref. 37. Copyright 2020 John Wiley \& Sons. (c) Fluorescence spectra of C153@Zr-NDC in a diethyl ether suspension. Increasing concentrations of $\mathrm{C} 153$ dye produces an increase in intensity of the $525 \mathrm{~nm}$ band (emission of C153) but a decrease of the $400 \mathrm{~nm}$ band (emission of Zr-NDC MOF). Adapted with permission from ref. 85. Copyright 2015 Royal Society of Chemistry.

Another important parameter is the luminescence quantum yield $(\Phi)$ of the LG@MOF composites, which is defined as the ratio of the number of photons emitted to the number of photons absorbed. The $\Phi$ value can be measured by using an integrating sphere coupled to a fluorometer and it will be of great importance not only to have an approximate idea of the ability to emit light of the LG@MOF system, but also it is an important correction factor for determination of other parameters, such as the Förster energy transfer efficiency (see Section 5.1) and fluorescence quenching. ${ }^{95}$ Both techniques, and especially the fluorescence spectroscopy, are therefore indispensable for the characterization of such excited-states processes and for the further integration of these materials in advanced technologies such as solid-state lighting or optical sensors (see Section 6). However, despite the great advantages evidenced above, these techniques will provide information of the presence of guests, but generally not a confirmation of their encapsulation within the MOF. The limitation lies in the fact that the signal will be equally detected no matter if the guests are on the surface, encapsulated in the pores, entrapped in the crystals, or a combination of some, or all, of the above locations. It is worth emphasizing that the fluorescence measurements can be complimented with time-resolved emission spectroscopy like time-correlated single photon counting (TCSPC) or fs-up-conversion techniques (see Section 5.4). Indeed, these time- 
resolved techniques are powerful tools to further unravel excited-state phenomena such as energy-, charge- or proton-transfer mechanisms as well as host-guest interactions.

\subsection{Nuclear Magnetic Resonance (NMR) Spectroscopy}

Another approach to establish the amount of luminescent guest confined in the MOFs is by digesting the LG@MOF composite (using acids or other solvents) and recording the solution ${ }^{1} \mathrm{H}$ NMR spectra. ${ }^{31,77,84,96-98}$ The molar ratio between the organic linker and the luminescent dye can be attained through the integral area of the ${ }^{1} \mathrm{H}$ NMR peaks. However, it is surprisingly frequent to find that in some reports there is a lack of information on how the weight percentage or the number of guest molecules were calculated. To cite a couple of examples, it was described that in the systems: $\mathrm{BI} @ \mathrm{~Pb}_{2}(\mathrm{TCPP}) \cdot 4 \mathrm{DMF}(\mathrm{BI}=$ benzylidene imidazolinone $)$ and C460@Eu $\mathrm{Eu}_{\mathrm{x}} \mathrm{Tb}_{\mathrm{y}} \mathrm{BPT}(\mathrm{C} 460=$ Coumarin 460$)$ there was one BI guest molecule per two TCPP4units and 1.02 wt.\% of C460 encapsulated in each MOF, without any additional information given on how this value was precisely determined. ${ }^{77,96}$ On the other hand, a more detailed study clearly explains the number of anthracene (ANT) molecules encapsulated in the cages of the ZIF-8. Firstly, the authors calculated the molar ratio between the organic linker (mIm) and the guest based on the integral area which was ANT/mIm $=1 / 3.37$. Then, knowing that 1 unit cell of ZIF-8 contains 2 cages with $24 \mathrm{mIm}$ ligands, the loading amount was obtained by multiplying the ANT/mIm ratio by 12 . In this example, the loading amount was 3.6 anthracene molecules per cage. ${ }^{31}$ In a very recent example, Tao et al. estimated the amount of fluorescein (Fluo) dye encapsulated in the pores of the ZIF-8 MOF (fluorescein@ZIF-8 system, see Figure 9a-b) by adopting the solution ${ }^{1} \mathrm{H}$ NMR methodology elucidated above, and it was found that the increasing amount of fluorescein introduced during the synthesis process does correlate with the guest occupancy of the pores (from 1/3703 to 1/6 fluorescein/cages), see Figure 9cd. ${ }^{83}$ 
(a)

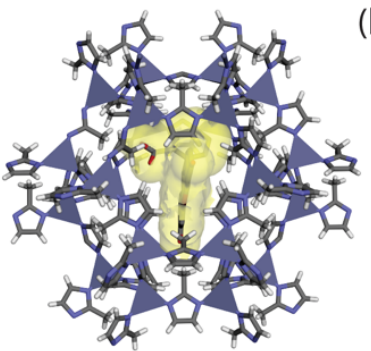

Fluo@ZIF-8

(c)

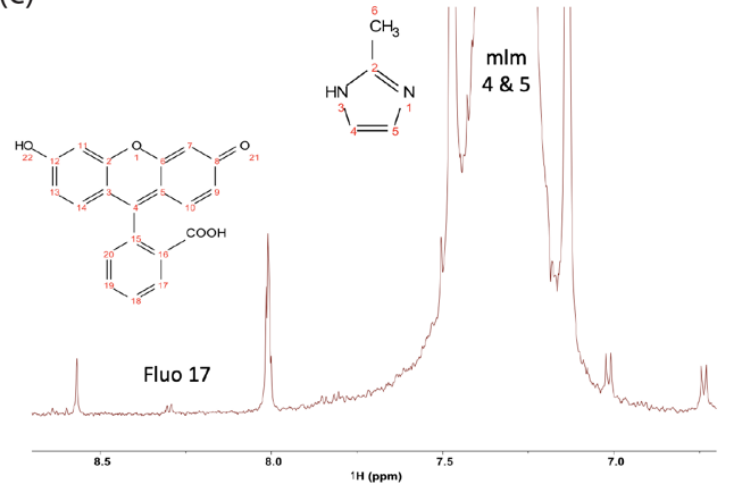

(b)

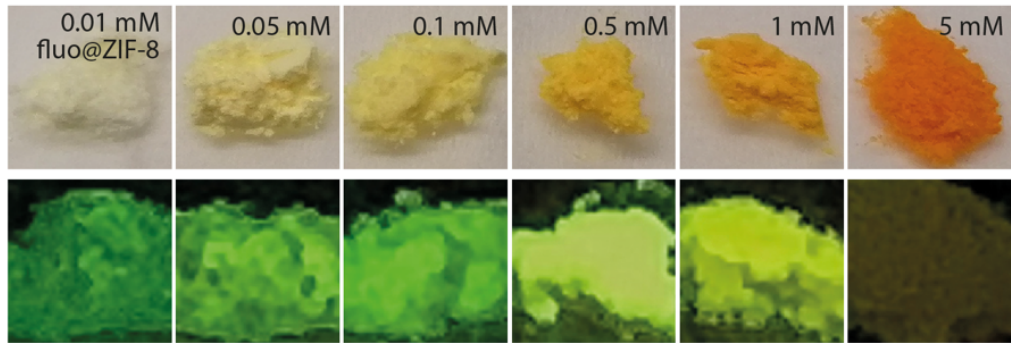

(d)

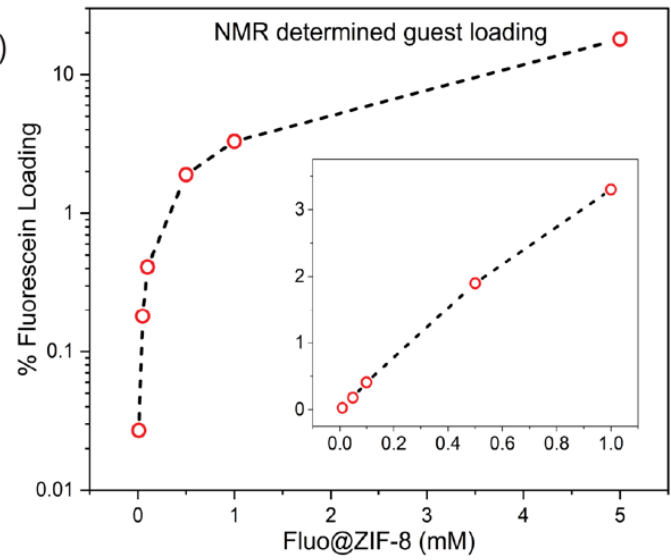

Figure 9. (a) Schematic representation of a fluorescein guest molecule encapsulated in the pore of a ZIF-8 sodalite cage, to yield the fluorescein@ZIF- 8 composite system. The purple tetrahedra are $\mathrm{ZnN}_{4}$. (b) Fluorescein@ZIF-8 samples with fluorescein guest concentrations of $0.01 \mathrm{mM}$ to $5 \mathrm{mM}$ viewed under natural light (top row) and their luminescence excited under a $365 \mathrm{~nm}$ UV lamp (bottom row). (c) Solution ${ }^{1} \mathrm{H}$ NMR spectrum of the $0.01 \mathrm{mM}$ fluorescein@ZIF-8 sample, where the guest/host peaks used for integration are indicated as Fluo and mIm, respectively. From this spectrum, the guest loading calculated is 1 fluorescein for every 3704 cages. (d) Fluorescein loading determined by NMR spectroscopy. The inset shows the loading amount plotted on a linear scale. Dashed lines are guide for the eye. Adapted from ref. 83. Copyright 2021 American Chemical Society.

However, and similarly to UV-vis spectroscopy, this methodology has some limitations. Firstly, even though the NMR technique is non-destructive by itself, the sample preparation step involving the digestion of the material turns it into a destructive methodology. Secondly and most important, this approach could determine the amount of guest but usually not revealing its location in the $\mathrm{MOF}$, hence the use of complimentary techniques will be required. Indeed, in the above case, ${ }^{31}$ a combination of $\mathrm{N}_{2}$ adsorption, ${ }^{1} \mathrm{H}$ NMR and thermogravimetric analyses (TGA) were required to further corroborate that the anthracene molecules were effectively confined into the cages of ZIF- $8 .{ }^{31}$ Despite this, in some very specific cases, ${ }^{1} \mathrm{H}$ NMR could reveal information on the guest location through indirect measurements. For example, the MOF Al-DBA (DBA =9,10-dibenzoate anthracene) contains capping acetates on the outer surface which exhibit a characteristic signal at $\sim 1.94 \mathrm{ppm}$ in the ${ }^{1} \mathrm{H}$ NMR spectra. ${ }^{97}$ 
After treating this MOF with the luminescent dye $\mathrm{RhB}$, the acetate signal disappears, indicating that $\mathrm{RhB}$ is replacing the capping agent in the outer surface of the MOF. ${ }^{97}$

\subsection{Elemental Analysis}

Elemental analysis (EA) is usually known as a technique that permits the qualitative identification and quantitative determination of elements such as $\mathrm{C}, \mathrm{N}, \mathrm{H}$, and $\mathrm{X}$ (where $\mathrm{X}=$ halogen, sulfur) of solid or fluid materials. EA is therefore a well-recognized technique to unveil the composition and purity of newly synthesized compounds, especially of organic systems. Moreover, in the last decades, EA is not only an outstanding tool to characterize the composition and purity of MOFs, ${ }^{76,93,98-100}$ but in some cases it also serves to identify and/or quantify different fluorescent guest compounds interacting with a MOF. ${ }^{101-103}$

For example, the presence of a diarylethene derivative chromophore in the ZJU-88 MOF was confirmed by the detection of $0.84 \%$ of sulfur element in the EA of the Guest@MOF composite. ${ }^{101}$ Another example analyzed the amount of N in a Ln@HPU-14 system, observing a decrease from $2.28 \%$ to $1.81 \%$ after the cation exchange process, which suggests that the $\left(\mathrm{CH}_{3}\right)_{2} \mathrm{NH}^{+}$cation was replaced by the fluorescent $\mathrm{Ln}^{3+}$ ions, reflecting that indirect EA measurements are also suitable for identifying the presence of guests in MOFs. ${ }^{104}$ On the other hand, it is also possible to quantify the amount of guests within MOFs, like in the case of the 4-(dicyanomethylene)-2-methyl-6-(4-dimethylaminostyryl)-4H-pyran (DCM) fluorophore loaded into two different MOFs (stilbene-MOF and IRMOF-8), where it was calculated to be $5.5 \%$ and $8.0 \%$, and the corresponding molecular formulae were expressed as $\mathrm{Zn}_{4} \mathrm{O}\left(\mathrm{C}_{12} \mathrm{H}_{6} \mathrm{O}_{4}\right)_{3}\left(\mathrm{C}_{19} \mathrm{H}_{16} \mathrm{~N}_{3} \mathrm{O}\right)_{0.18}$ and $\mathrm{Zn}_{4} \mathrm{O}\left(\mathrm{C}_{16} \mathrm{H}_{10} \mathrm{O}_{4}\right)_{3}\left(\mathrm{C}_{19} \mathrm{H}_{16} \mathrm{~N}_{3} \mathrm{O}\right)_{0.31}$, respectively. ${ }^{102}$

Similarly, in another study, the amount of $\mathrm{DASP}^{+}$((4-p-(dimethylamino)styryl)-1methylpyridinium) incorporated in the bio-MOF-100 was calculated using the EA obtained from 4 different batches of DASP $@$ @bio-MOF-100 composite. In this case, the authors provide a detailed description of the calculation to establish the $\mathrm{DASP}^{+}$concentration. Firstly, they assumed that the number of replaced cations is $n$, and thus, the chemical formulae for the host@guest compound was $\mathrm{Zn}_{8}\left(\mathrm{C}_{5} \mathrm{H}_{4} \mathrm{~N}_{5}\right)_{4}\left(\mathrm{C}_{14} \mathrm{H}_{8} \mathrm{O}_{4}\right)_{6} \mathrm{O}_{2} \cdot(4-n) \mathrm{Me}_{2} \mathrm{NH}_{2} \cdot n\left(\mathrm{C}_{16} \mathrm{H}_{19} \mathrm{~N}_{2}\right)$, where $n$ was determined by EA of $\mathrm{C}, \mathrm{H}$ and $\mathrm{N}$ atoms. The authors then used the following equation to calculate the concentration of $\mathrm{DASP}^{+}$in bio-MOF-100: $C_{\text {dye@MOFs }}=n Z /\left(N_{\mathrm{A}} V\right)$, where $Z$ is the number of formula units per cell (48), $V$ is the cell volume $\left(330,226 \AA^{3}\right)$ and $N_{\mathrm{A}}$ is the Avogadro's number. Applying this relationship, the concentration of DASP ${ }^{+}$for the 4 different batches was calculated to be $0.03,0.06,0.30$, and $0.44 \mathrm{~mol} / \mathrm{L} .{ }^{103}$ 
In addition to the conventional elemental analyzer technique, in which the CHNS analysis is accomplished by a combustion of the sample, there exist other techniques capable of detecting and quantifying different elements such as X-ray photoelectron spectroscopy (XPS), X-ray fluorescence (XRF), and inductively coupled plasma optical emission spectroscopy (ICP-OES). All these techniques have also been applied to recognize, and in some cases to quantify the elemental composition of different Guest@MOF materials. ${ }^{64,105-109}$ For instance, the presence of luminescent $\mathrm{Cu}$ nanoclusters (NCs) in ZIF-90 or MOF-5 has been detected by means of XPS, where the spectrum of the materials showed the peaks at $932 \mathrm{eV}$ and $952 \mathrm{eV}$ attributed to $\mathrm{Cu}_{2 \mathrm{p} 3 / 2}$ and $\mathrm{Cu}_{2 \mathrm{p} 1 / 2}$, which are the characteristic peaks of $\mathrm{Cu}^{0}$ (Figure 10). ${ }^{64,105}$ Alternatively, another study employed XRF to calculate the amount of $\mathrm{Ln}^{3+}$ ions incorporated in two defect-engineered MOFs (UiO-66-AB and MIL-53(Al)-AB), by estimating the ratio between the metal ions of the MOF and the Ln ones, this being $1 / 1(\mathrm{Zr} / \mathrm{Ln})$ in the case of UiO66-AB and $0.5 / 1(\mathrm{Al} / \mathrm{Ln})$ for the MIL-53(Al)-AB system. ${ }^{107}$ Other works reported on the combination of ICP-OES and conventional EA techniques for characterizing the chemical composition of different Ln@bio-MOF-1 composites. ${ }^{108,109}$ Indeed, from these experiments they could extract the molar ratios of $\mathrm{Zn}^{2+}: \mathrm{Tb}^{3+}: \mathrm{Eu}^{3+}: \mathrm{C}: \mathrm{H}: \mathrm{N}$ for three $\mathrm{Ln} @$ bio-MOF-1 materials containing different amounts of $\mathrm{Ln}$ ions, which were found to be 1:0.903:0.098:16.20:19.22:3.63 for $\mathrm{Tb}_{0.9} \mathrm{Eu}_{0.1} @$ bio-MOF-1, 1:0.987:0.011:16.14:19.11:3.60 for $\mathrm{Tb}_{0.99} \mathrm{Eu}_{0.01} @$ bio-MOF-1, and 1:0.9993:0.0008:16.31:19.15:3.65 for Tb0999 $\mathrm{Eu}_{0.001} @$ bioMOF-1. 109

Despite the ability to detect and quantify different elements and therefore to confirm the presence of different fluorescent guests in MOF materials, the EA techniques described herein share the same limitation to the other techniques described above, and it is the inability to pinpoint the precise location of the guests in the MOF hosts.
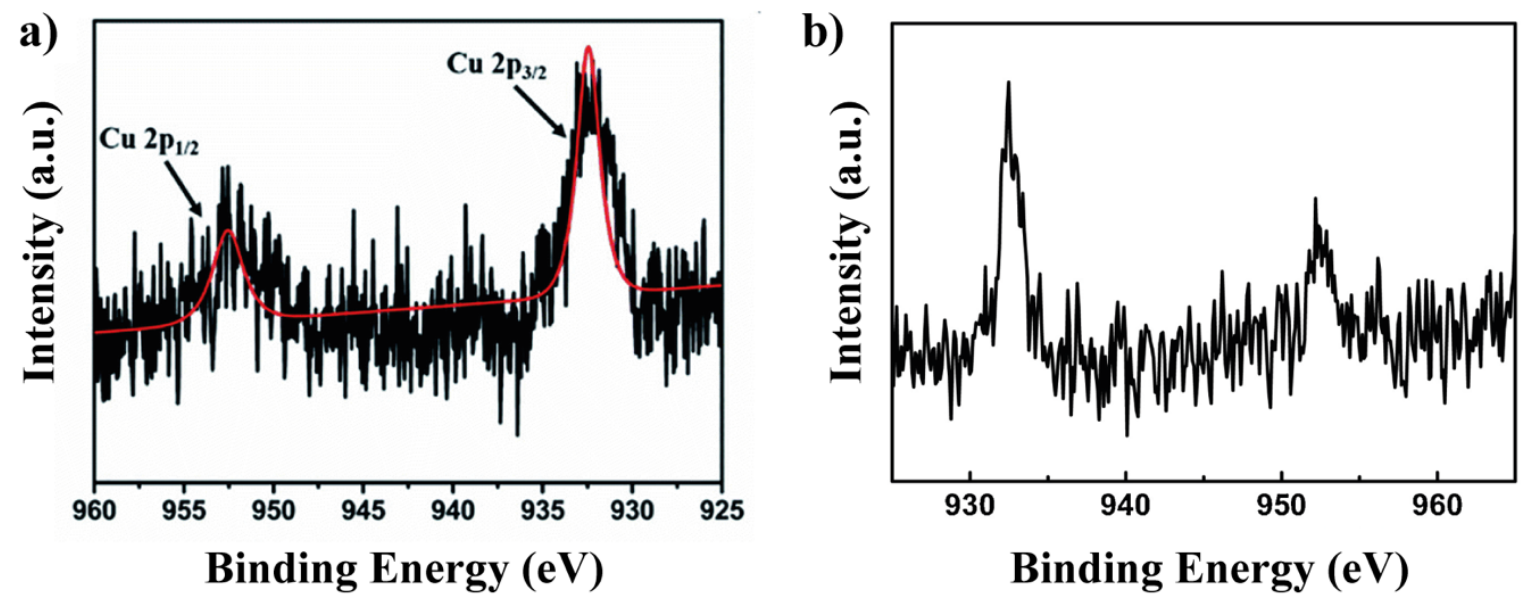
Figure 10. XPS spectrum of (a) Cu NCs@GSH/MOF-5 [GSH = glutathione]. Adapted with permission from ref. 64. Copyright 2018 Royal Society of Chemistry. and (b) CuNCs$\mathrm{Al}^{3+} / \mathrm{ZIF}-90$ composites in the $\mathrm{Cu} 2 \mathrm{p}$ region. Adapted with permission from ref. 105. Copyright 2020 Elsevier.

\subsection{Vibrational Spectroscopy (IR, Raman)}

Fourier transform infrared (FTIR) spectroscopy is an analytical technique that employs infrared radiation to probe the rotational and/or vibrational transitions of the molecules, resulting in a spectrum which is a unique fingerprint of the sample (especially in mid-IR regime), making it a versatile and effective technique for the identification of organic molecules or polymers, among others. FTIR spectroscopy is probably, together with the UVvis/fluorescence spectroscopy, the most exploited technique for the characterization of LG@MOF materials. ${ }^{67,68,70,110-115}$ The simultaneous coexistence of the IR bands characteristics of the fluorophores and the MOF usually serves to identify the presence of the former and to prove the robustness of the latter upon encapsulation, all at once. ${ }^{67,68,70,110-115}$ Numerous and diverse examples have been reported in this sense, such as the presence of different carbon quantum dots (CDs) or metal-organic complexes (like $\mathrm{Gaq}_{3}$ ) confined in the ZIF-8 matrix, see Figure 11a-d. ${ }^{37,67,68,111}$ In those systems, the FTIR spectrum is a combination of the typical IR bands of ZIF-8 and new bands of the luminescent guests like those of $\mathrm{Gaq}_{3}$ (Figure 11c) or a broad band at $\sim 3420 \mathrm{~cm}^{-1}$ attributed to the $\mathrm{O}-\mathrm{H}$ stretching vibration of the CDs (Figure 11d), reflecting the presence of the fluorophores and at the same time the chemical stability of the ZIF-8 after their incorporation. ${ }^{37,67,68,111}$ Similarly, the presence of inorganic perovskite nanocrystals (NCs) in different MOF hosts have been identified by the simultaneous existence of IR bands associated to the specific MOF and to the perovskite NCs, especially the bands at 2924 and $2853 \mathrm{~cm}^{-1}$, which are the characteristics signals of the $\mathrm{C}-\mathrm{H}$ symmetric and asymmetric stretching vibrations of oleylamine and oleic acid capping agents present in perovskite NCs (Figure 11e). ${ }^{70,114}$ The same approach was also followed for the identification of organic dyes like fluorescein in the UiO-66 MOF, where the bands at $1638 \mathrm{~cm}^{-1}$ and 1643 $\mathrm{cm}^{-1}$ were assigned to the $\mathrm{C}=\mathrm{O}$ vibrations in the benzoquinone structure of the fluorescein molecule. ${ }^{110}$ 
a)

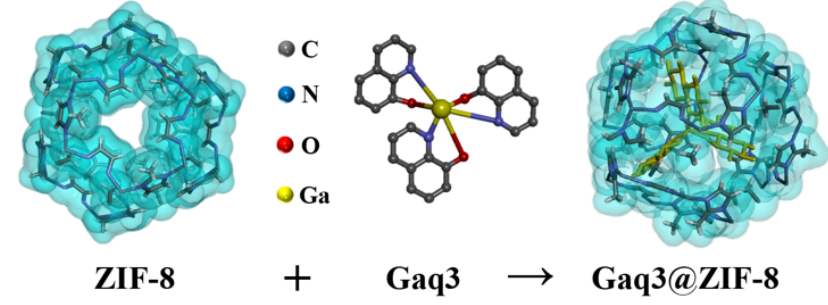

b)

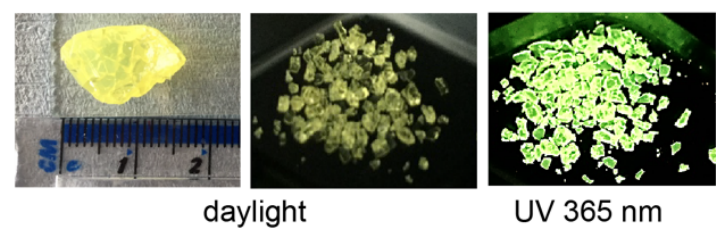

d)

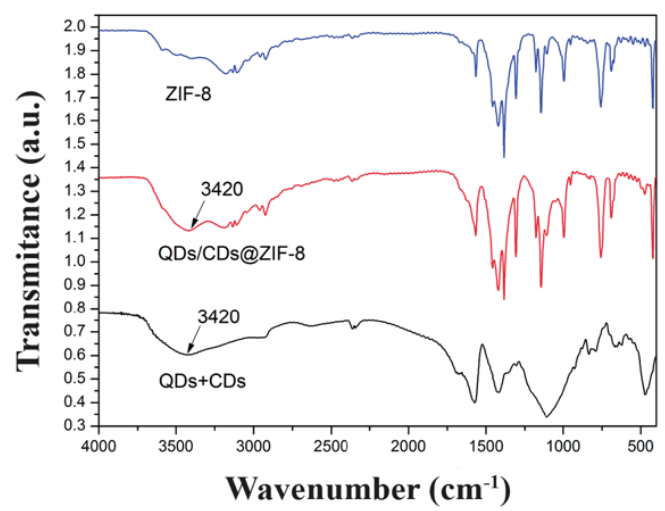

c)

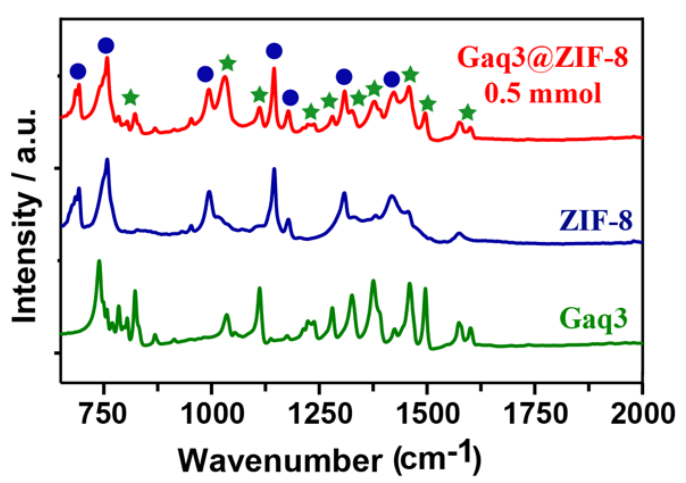

e)

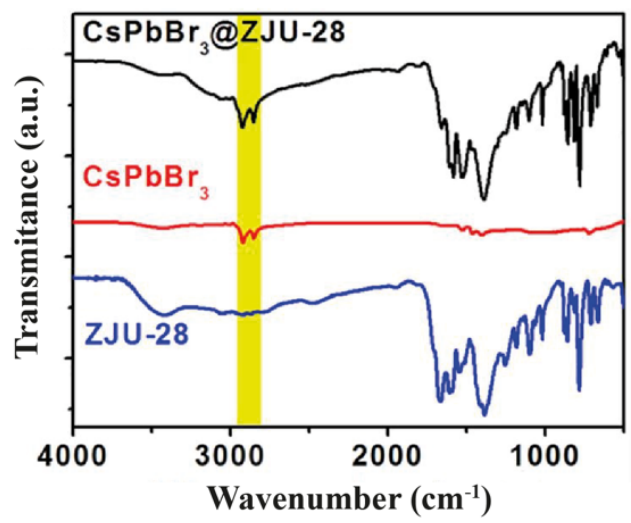

Figure 11. (a) Representation of the chemical structures of ZIF-8 host, Gaq 3 guest and the Gaq $_{3} @$ ZIF-8 composite material. (b) A large and small fragmented monoliths of Gaq $3 @ Z$ ZIF8 viewed under daylight and its greenish emission under 365-nm UV lamp. (c) FTIR spectra of Gaq3@ZIF-8 showing a combination of the vibrational bands that are characteristic of Gaq 3 guest (green stars) and ZIF-8 host (blue dots). Adapted from ref. 37. Copyright 2020 John Wiley \& Sons. (d) ZIF-8, QDs/CDs@ZIF-8 and a mixture of QDs and CDs, adapted with permission from ref. 111. Copyright 2017 Royal Society of Chemistry. and (e) FTIR spectra of ZJU-28, $\mathrm{CsPbBr}_{3}$ and $\mathrm{CsPbBr}_{3} @ \mathrm{ZJU}-28$, adapted with permission from ref. 114. Copyright 2020 Elsevier.

While FTIR is an outstanding technique for the chemical identification of guest fluorophores in the MOF materials, it is not so suitable to quantify the amount of guest neither to differentiate the position of the guest in the MOF (i.e., encapsulated in the pores, embedded in the crystal, or adsorbed on the surface). An excellent example of this is the protein-embedded cytochrome $\mathrm{c}$ (Cyt $c$ ) in ZIF-8 MOF. ${ }^{116}$ In spite of the detection of the band at $1664 \mathrm{~cm}^{-1}$ assigned to the stretching modes of double bonds and carbonyls in Cyt $c$, the authors demonstrated through a calcination process that the Cyt $c$ were mostly deposited on the MOF surface rather than being encapsulated in the pores of ZIF-8. ${ }^{116}$ On the other hand, in some very specific cases, the FTIR results can provide information that may suggest the location of the guest. For example, the encapsulation of RhB and fluorescein dyes in the ZIF-8 MOF leads to 
a suppression of their IR band at $1600 \mathrm{~cm}^{-1}$ owing to an increase in their framework rigidity, suggesting that both molecules are well incorporated in the pores of the MOF. ${ }^{82}$ However, some precautions must be taken with these statements, like in the case of the $\mathrm{Cu}$ nanocrystals (CuNCs) incorporated into ZIF-90, where the authors observed the lack of the bands corresponding to CuNCs and CuNCs $+\mathrm{Al}^{3+}$ at $2080 \mathrm{~cm}^{-1}$ and $700 \mathrm{~cm}^{-1}$ and attributed this phenomenon to the encapsulation of the CuNCs inside the pores, which is very unlikely as the ZIF-90 pore size is $\sim 3.5 \AA$, ${ }^{117}$ while the diameter of the CuNCs is of $5.5 \mathrm{~nm}$. ${ }^{105}$ A recent study on the fluorescein@ZIF-8 system has employed a combination of ATR-FTIR measurements and $a b$ initio calculations by density functional theory (DFT) to study the phenomenon of guest-host interactions. ${ }^{83}$ The comparison between the simulated IR spectra and experimental observations support the notion that the fluorescein guest can be encapsulated in the cage of the ZIF-8. Notably, a shift in the IR vibrational peaks has been shown to be the result of guest confinement in the cage and the interaction with the host framework.

Although less widely utilized, Raman vibrational spectroscopy could also be a powerful tool for the characterization of LG@MOF systems. For example, vibrational data measured by Raman spectroscopy show similar spectra of the pristine ZIF-8 and the latter containing the fluorescent ZnQ complex, suggesting that ZIF-8 MOF retained its pristine form after the encapsulation of the fluorophore. ${ }^{32}$ A more interesting example is given by the encapsulation of the same $\mathrm{ZnQ}$ complex in the Zn-based OX-1 MOF. ${ }^{35}$ In this case, Raman spectroscopy provides further insights into symmetry alterations of the guest by the confinement effect. The analysis shows that the doubly degenerate Raman modes at $\sim 504 \mathrm{~cm}^{-1}$ and $514 \mathrm{~cm}^{-1}$ (attributed to the skeletal in-plane bending vibrations) of ZnQ turn into a single band at $\sim 508 \mathrm{~cm}^{-1}$ when the fluorophore is encapsulated, reflecting a higher structural symmetry. ${ }^{35}$ Although Raman spectroscopy is a notable complimentary technique, it presents similar limitations to those described for FTIR.

\subsection{Electron Microscopy (SEM, TEM, EDX, FIB)}

The scanning electron microscopy (SEM) is a technique that uses a focused beam of electrons to scan the surface of the samples generating an image of the topography usually detecting the secondary electrons emitted by the excited atoms. Although SEM is widely employed for characterizing the morphology of MOF crystals, it does not usually provide key information on the encapsulation of guests. Indeed, most of the examples draw their conclusion pertaining to the morphology of the MOF crystals before and after the encapsulation of guests. 
For instance, the SEM images of a stilbene-MOF or the bio-MOF-1 after the encapsulation of $\mathrm{DCM}$ or RhB dyes show no changes in the morphology, reflecting the stability of both materials after the guest encapsulation step. ${ }^{94,102}$ On the other hand, there exist examples where the encapsulation of guest induces changes in the morphology, but not in the MOF periodic structure. The encapsulation of carbon dots and curcumin into ZIF-8 generates spherical ZIF8 structures with a rough surface, very different from the regular smooth dodecahedron crystals of pristine ZIF-8. ${ }^{118}$ Although one might be tempted to correlate this morphological change to an effective guest encapsulation, other parameters like changes in the synthetic methodology or the chemical properties of the guests should be considered and therefore additional proofs are required to corroborate the guest encapsulation.

Notably, the SEM instrument can incorporate multiple detectors to collect the secondary electrons (SE), back-scattered electrons (BSE), or the characteristics X-ray. The latter derives into a technique known as energy dispersive X-ray spectroscopy (EDS, EDX or EDXS) which is a type of analytical technique used for the elemental analysis of samples. SEM-EDX has been used for mapping the distribution of guest interacting with MOFs, especially those guest composed of metal-based nanocrystals. ${ }^{26,64,113,114,119-122}$ There are many examples of perovskite nanocrystals interacting with different MOFs, where a mapping distribution of elements like $\mathrm{Pb}, \mathrm{Br}$ or $\mathrm{Cs}$ (typical components of hybrid and all inorganic perovskites) indicates a homogeneous distribution of the perovskites over the MOF crystals (Figure 12). ${ }^{26,119-122}$ Similarly, the homogenous distribution of $\mathrm{Cu}$ nanocrystals in ZIF-8 and MOF-5 has been analyzed based on the SEM-EDX images of those composite materials. ${ }^{64,113}$ Even though SEM-EDX can shed some light on the guest distribution, there are some limitations such as the difficulty to detect luminescent organic dyes, which are typically composed of light atoms like $\mathrm{C}, \mathrm{O}$, and $\mathrm{N}$ elements, very similar to the elements that form the organic linkers. Another drawback is the fact that the depth of penetration is not very high, and therefore it is very plausible that the elements detected from the guest are very close or adhered on the surface of MOF crystals, hindering a precise determination of the location of the guests in the MOF host. Focused ion-beam (FIB) milling via $\mathrm{Ga}^{+}$ions has also been employed in conjunction with SEM to remove thin consecutive slices of MOF composite materials, which enabled 3-D reconstructions to study interfaces, volumetric defects, and phase distributions at the micro- and nanoscale. ${ }^{123-127}$ Naturally, this FIB-SEM approach can be extended to enable microstructural examination of LG@MOF systems (e.g., to yield 3-D reconstruction of coreshell materials), provided caution is taken to minimize gallium ion beam damage ${ }^{128}$ to fluorophores and organic moieties. 

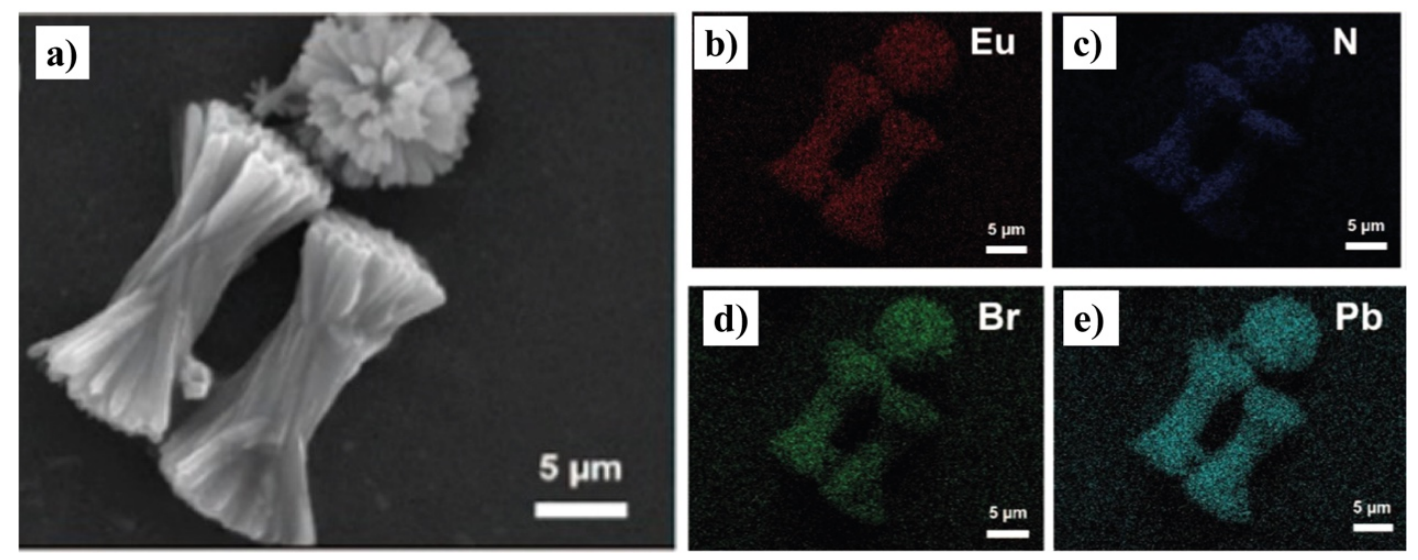

Figure 12. (a) SEM image of EuBTC microcrystals, and the elemental mapping diagrams of (b) $\mathrm{Eu}$, (c) $\mathrm{N}$, (d) $\mathrm{Br}$, and (e) $\mathrm{Pb}$ in the $\mathrm{CH}_{3} \mathrm{NH}_{3} \mathrm{PbBr}_{3} @$ EuBTC composite. Adapted with permission from ref. 120. Copyright 2018 American Chemical Society.

Akin to an SEM, a transmission electron microscope (TEM) also uses a beam of electrons, but in this case, the beam is transmitted through the sample and then magnified and focused by the objective lens onto an imaging device (fluorescent screen) or a charge coupled device (CCD) camera. The maximum resolution of a TEM is considerably greater than an SEM, revealing details at an atomistic scale as low as 1-2 A.. In the field of LG@MOF, TEM has sometimes been used to unveil changes in the morphology of the MOF after its interaction with the guest. For example, the interaction of a perylene derivative with the ZIF-8 MOF produces a change in the morphology (from tetrakaidecahedra to irregular spheres) and the size of the crystals, ascribed to a possible competition of the perylene and the 2-methylimidazole linker for binding the $\mathrm{Zn}^{2+}$ ions. ${ }^{64,113}$ However, the most extended use of TEM in this field is to visualize the distribution of quantum dots and nanocrystals in different MOF systems. There are numerous examples where TEM images unveil the presence and in some cases, the position of carbon quantum dots (CDs) in different MOF matrices. ${ }^{67,112,129,130}$ For example, the presence of CDs of about $10 \mathrm{~nm}$ in the surface of two different Zn-based and Ln-based MOFs has been demonstrated (Figure 13a-b). ${ }^{112,129}$ In other cases, determination of the location of the CDs is not so trivial, like the formation of CDs after calcination of ZIF-8, where the CDs of a size around $4 \mathrm{~nm}$ give the impression to be entrapped in the ZIF-8 framework (Figure 13c-d). ${ }^{67} \mathrm{In}$ other composites, the detection of the CDs is not possible, like in the case of an Eu-MOF system where the authors described that the surface of the rod crystals is smooth, so they have suggested but not confirmed that the CDs may not be attached onto the surface. ${ }^{130}$ Likewise, the TEM technique has been employed to probe the distribution of different quantum dots (e.g., $\mathrm{ZnS}, \mathrm{CdS}, \mathrm{CdTe}$ ) within the different MOF frameworks. ${ }^{34,115,131}$ 

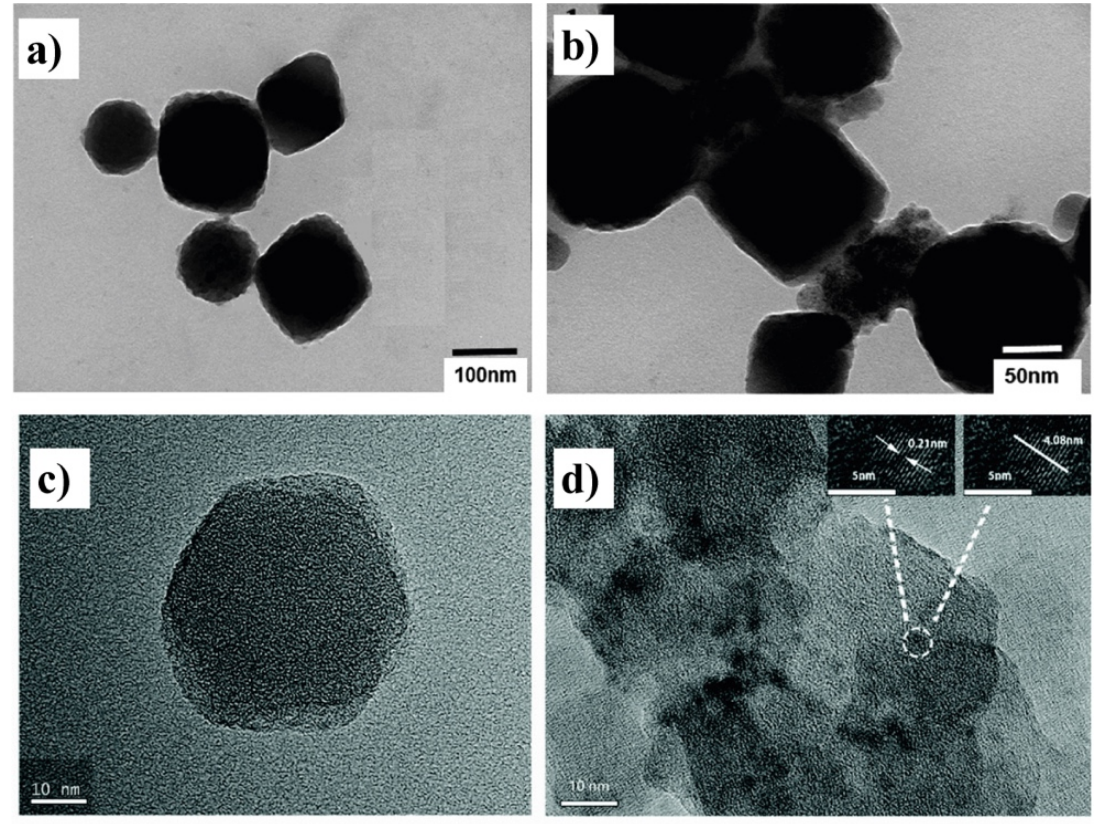

Figure 13. TEM micrographs of (a-b) CDs/Zn-MOFs. Adapted with permission from ref. 129. Copyright 2020 Elsevier. (c) ZIF-8, and (d) CDs@ZIF-8. Adapted with permission from ref. 67. Copyright 2018 Royal Society of Chemistry.

Comparable to SEM, TEM images can be combined with EDX analysis for mapping the elemental distribution of a nanomaterial. Even though this is not so frequently employed for LG@MOFs, still some examples can be found. For example, while (high-resolution) HRTEM images of a composite comprising $\mathrm{Cu}$ nanoclusters and a ZIF-8 matrix does not provide any evidence of the nanoclusters distribution, an EDX elemental map clearly reveal a homogenous distribution of $\mathrm{Cu}$ atoms all over the ZIF-8 crystals, suggesting a homogenous dispersion of the nanoclusters (Figure 14). ${ }^{132}$ However, and in addition to the problems related to the location of the guests previously mentioned, and the fact that generally it will not be possible to detect small organic dyes in a MOF structure, TEM also presents major drawbacks like an extensive sample preparation, as it must be thin enough to transmit electrons, making this technique time consuming. Moreover, there is always a risk of sample irradiation damage by the intense electron beam, reducing the applicability of this technique. Above notwithstanding, the recent advances in HRTEM at cryogenic temperatures (cryo-TEM) and low-dose TEM have opened the door to probe the local structures of MOFs at atomic resolution, ${ }^{133}$ as exemplified by direct imaging of host-guest structures within ZIF-8 (herein guest is adsorbed $\mathrm{CO}_{2}$, see Figure 15), ${ }^{134}$ and local structural analysis of the luminescent CsPbX3@MIL-101 
composites. ${ }^{135}$ These atomic-resolution imaging techniques have the potential to be applied to pinpoint exact positions of fluorophore molecules confined in the LG@MOF systems.
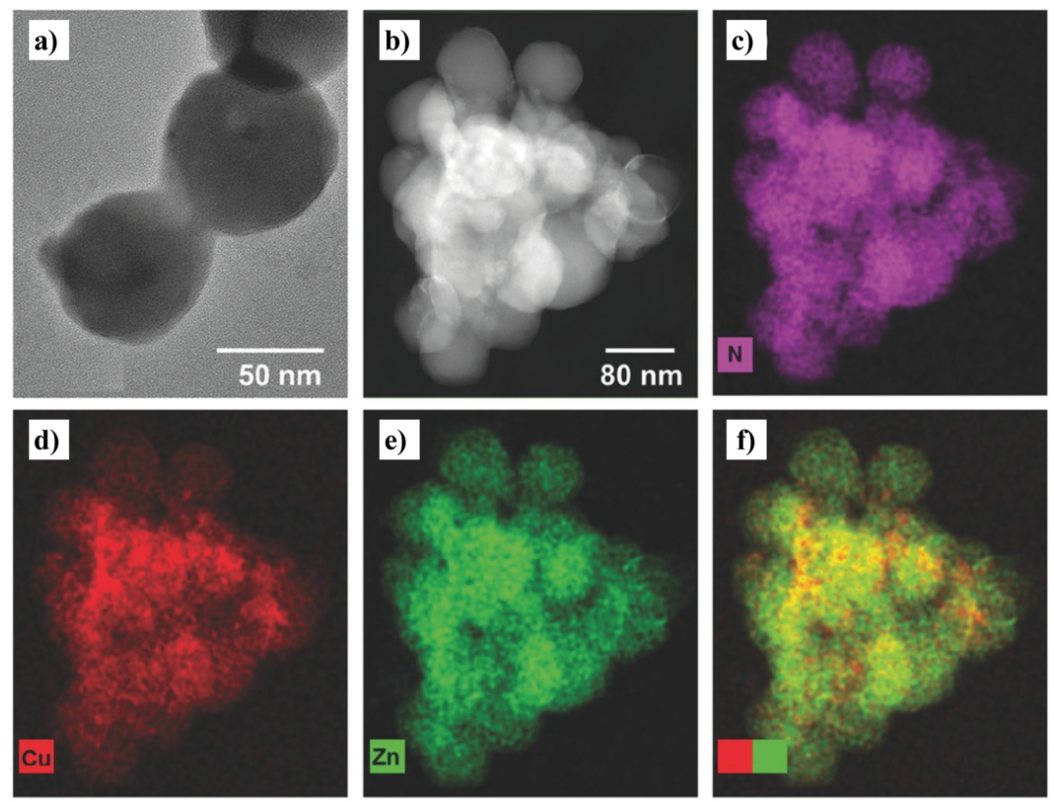

Figure 14. (a) HRTEM and (b) HAADF TEM images of Cu NC/ZIF-8 composites. (c-e) EDS elemental mapping $\mathrm{N}, \mathrm{Cu}$, and $\mathrm{Zn}$ (indicated in the figure). (f) Superimposed EDS images of $\mathrm{Cu}$ (red) and $\mathrm{Zn}$ (green) elements. Adapted with permission from ref. 132. Copyright 2017 John Wiley \& Sons.

a)

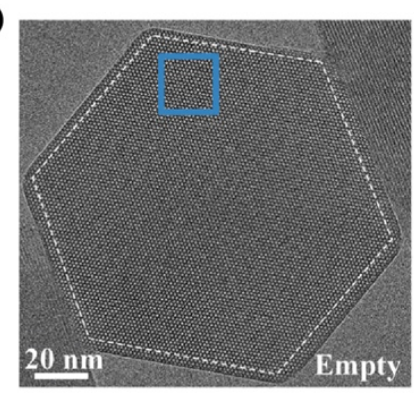

d)

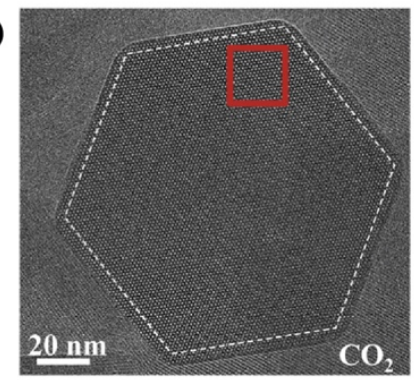

b)

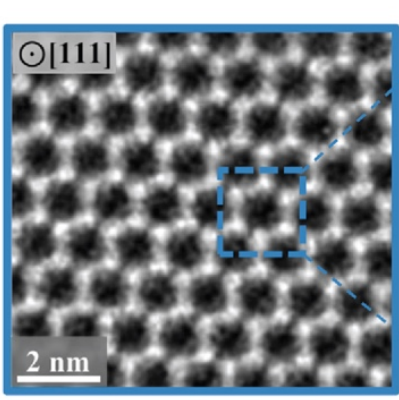

e)

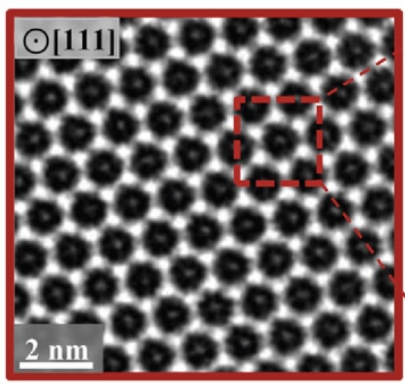

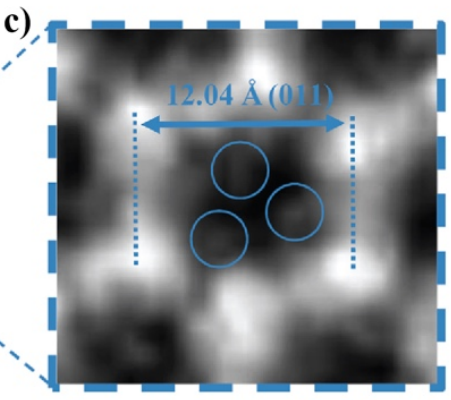

f)

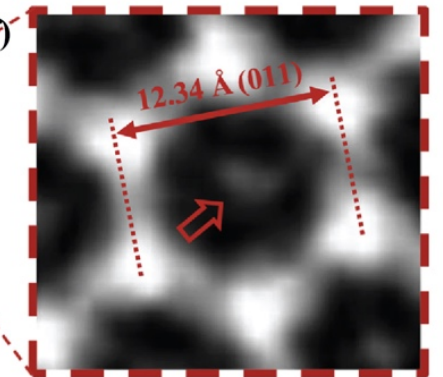

Figure 15. (a, d) Cryo-EM images of the evacuated and $\mathrm{CO}_{2}$-filled ZIF8 crystals, respectively, viewed along the $\langle 111\rangle$ crystallographic axis. (b, e) Contrast transfer function (CTF)-corrected denoised images corresponding to the blue and red boxes on the left panels (a-b), where the bright regions indicate to the mass density. (c, f) Magnified images of a unit cell of ZIF8, where the blue circled regions may correspond to the mIm linkers, while the red arrow possibly indicates position of an adsorbed $\mathrm{CO}_{2}$ in the sodalite cage. Adapted with permission from ref. 134. Copyright 2019 Elsevier. 


\subsection{Thermogravimetric Analysis (TGA)}

Thermogravimetric analysis (TGA) is a routine technique for materials characterization in which the mass of the sample is measured over time while the temperature changes. This usually provides information about the phase transition, the adsorption/desorption of molecules (e.g., gas or solvent molecules), or the thermal stability of a material. Indeed, most of the examples reported hitherto in the field of LG@MOFs exploited this technique to ascertain the thermal stability of those composite materials and the presence of solvent molecules..$^{50,51,77,78,97,115,136,137}$ However, there are many other interesting works that leverage the TGA technique to pinpoint the mass percentage of the guest or to appraise whether it is entrapped into the MOF structure.

The loading fraction of RhB dye (ranging from $0.89 \%$ to $4.0 \%$ ) into DUT-52, ${ }^{138}$ the loaded mole ratio of Eosin $\mathrm{Y}$ dye $(0.16 \%$ to $3.66 \%)$ in DUT-52, ${ }^{139}$ the content of 4aminonaphthalimide $(0.052 \%)$ in a $\mathrm{Ln}$-based $\mathrm{MOF}^{140}$ or the weight percentage of anthracene $(32 \%)$ in ZIF- $8^{31}$ are archetypal examples of the calculation of the amount of dye in MOFs by employing TGA. Other works have used the results obtained from TGA to claim that the dyes are effectively encapsulated in the framework. ${ }^{94,141,142}$ To this end, an enhancement of the thermal stability of the guest is usually employed to justify guest encapsulation. ${ }^{94}$ However, it is worth remarking that similar effect could be observed when the guest is strongly adsorbed on the surface of the material, so the overall thermal enhancement could be a combination of molecules adsorbed and entrapped in the MOF. Because of that, we consider that it is very risky to assert a guest encapsulation taking into account only the results obtained from TGA, while the combination of the latter with other complementary techniques (such as NMR and FTIR) could more accurately unveil the location of the guest in the framework as reported for the anthracene@ZIF-8 and fluorescein@ZIF-8 systems. ${ }^{31,83}$

\subsection{Other promising but less explored techniques}

\subsubsection{Confocal laser scanning microscopy (CLSM)}

A confocal laser scanning microscope (CLSM) is an optical instrument for imaging luminescent samples with an increased optical resolution (compared to fluorescence microscopes) owing to a spatial pinhole that blocks light that is out of focus. ${ }^{143}$ The use of this technique is well spread in the biological and medical research areas, however, a few studies 
employing CLSM in the field of LG@MOFs are largely focused on the analysis of guest distribution over the MOF crystals. Typical examples have shown a homogeneous distribution of different types of fluorescent dye (e.g., $\mathrm{RhB}$, nile red, porous organic nanosheets, 4-(pdimethylaminostyryl-1-methylpuridinium) over a number of different MOF crystals $(\mathrm{Cd}(\mathrm{m}-$ bdc)(bIm), a 2D Al-MOF, ZIF-8, bio-MOF-1, ZJU-28). ${ }^{42,75,142,144,145}$ Some of these examples attributed the homogenous distribution of the luminescent guest to an effective encapsulation in the MOF pores. However, it is important to remark that, on one side, the resolution of the confocal microscope is limited (among other things) by the excitation wavelength, providing a typical resolution of $180 \mathrm{~nm}$ laterally and $500 \mathrm{~nm}$ axially, and therefore, it will not be possible to pinpoint a homogeneous distribution in that $\mathrm{nm}$ range; and on the other side, it will be a very difficult task to distinguish whether the emission detected is originated only from the guests adsorbed on the surface, encapsulated in the pores, or a mixture of both. In this sense, a good example is one reported by Yan and coworkers where the encapsulation of DCM dye into a stilbene-MOF is proven by the homogeneous dispersion of the guest shown in different luminescent micrographs recorded in the $z$-axis direction (Figure 16a) and by scanning the $x y$ plane in the $z$-axis direction (Figure 16b).${ }^{102}$ However, even in this outstanding visual example, it is not possible to completely rule out that part of the emission observed may arise from molecules adsorbed on the surface of the MOF.

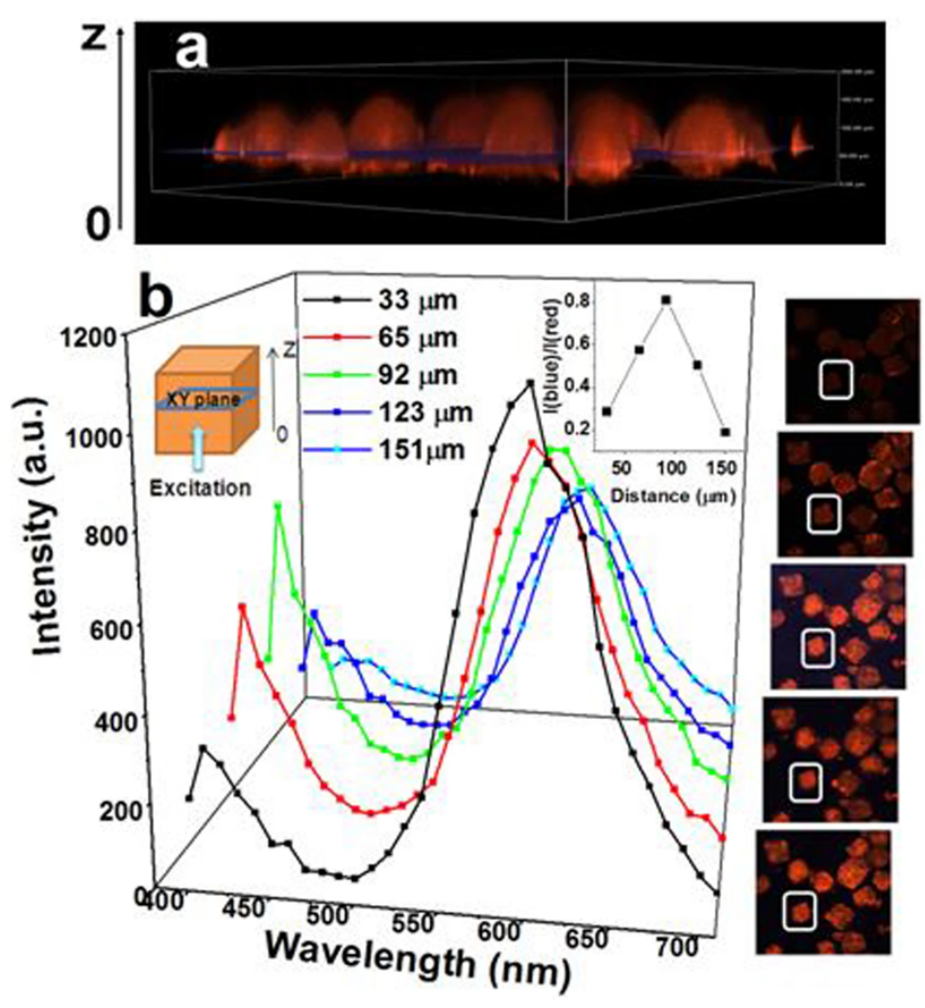


Figure 16. (a) 3D fluorescence image of DCM@stilbene-MOF. (b) Emission spectra and intensity ratios of blue to red emissions (inset) of a DCM@stilbene-MOF single crystal. The inset photographs are the fluorescence images in focal planes at different distances $(33,65,92$, 123 and $151 \mu \mathrm{m})$ from the excitation laser $(372 \mathrm{~nm})$. Adapted with permission from ref. 102 . Copyright 2014 Nature Publishing Group.

On the other hand, in some cases where the size of the MOF crystal is of the tens to hundreds of micrometer scale, it would be possible to determine with better precision the location of the guests within the MOF host. ${ }^{146,147}$ For instance, the confocal imaging in the $z$-axis of rhodamine $6 \mathrm{G}(\mathrm{R} 6 \mathrm{G})$ within large micron-sized In-BTB single crystals (where BTB $=1,3,5$-benzenetribenzoate) revealed that, the $\mathrm{R} 6 \mathrm{G}$ dye molecules were located mostly at the periphery of the In-BTB MOF, and that they cannot fully penetrate into the interior of the crystal, see Figure 17. ${ }^{147}$ From a sliced image of the crystal, the authors estimated a depth penetration of only $5-13 \mu \mathrm{m}$ for a crystal size of $\sim 100 \mu \mathrm{m}$. In another interesting example, confocal microscopy was proven to be a powerful tool to unveil the presence of BODIPY (boron-dipyrromethene) and resorufin dyes within a cyclodextrin-based MOF (crystal size $\sim 50 \mu \mathrm{m}$ ), where other techniques such as ${ }^{1} \mathrm{H}$ NMR and SCXRD failed to provide the missing evidence ${ }^{147}$ Moreover, it was possible to pinpoint the location of both dyes on the cyclodextrinbased MOF. While the BODIPY dye was accumulated on the edges of the MOF crystal owing to its bigger size (restrictive confinement effect), the smaller resorufin dye was able to permeate within the crystal fracture planes of the MOF, which presumably are higher polarity regions. ${ }^{147}$ 


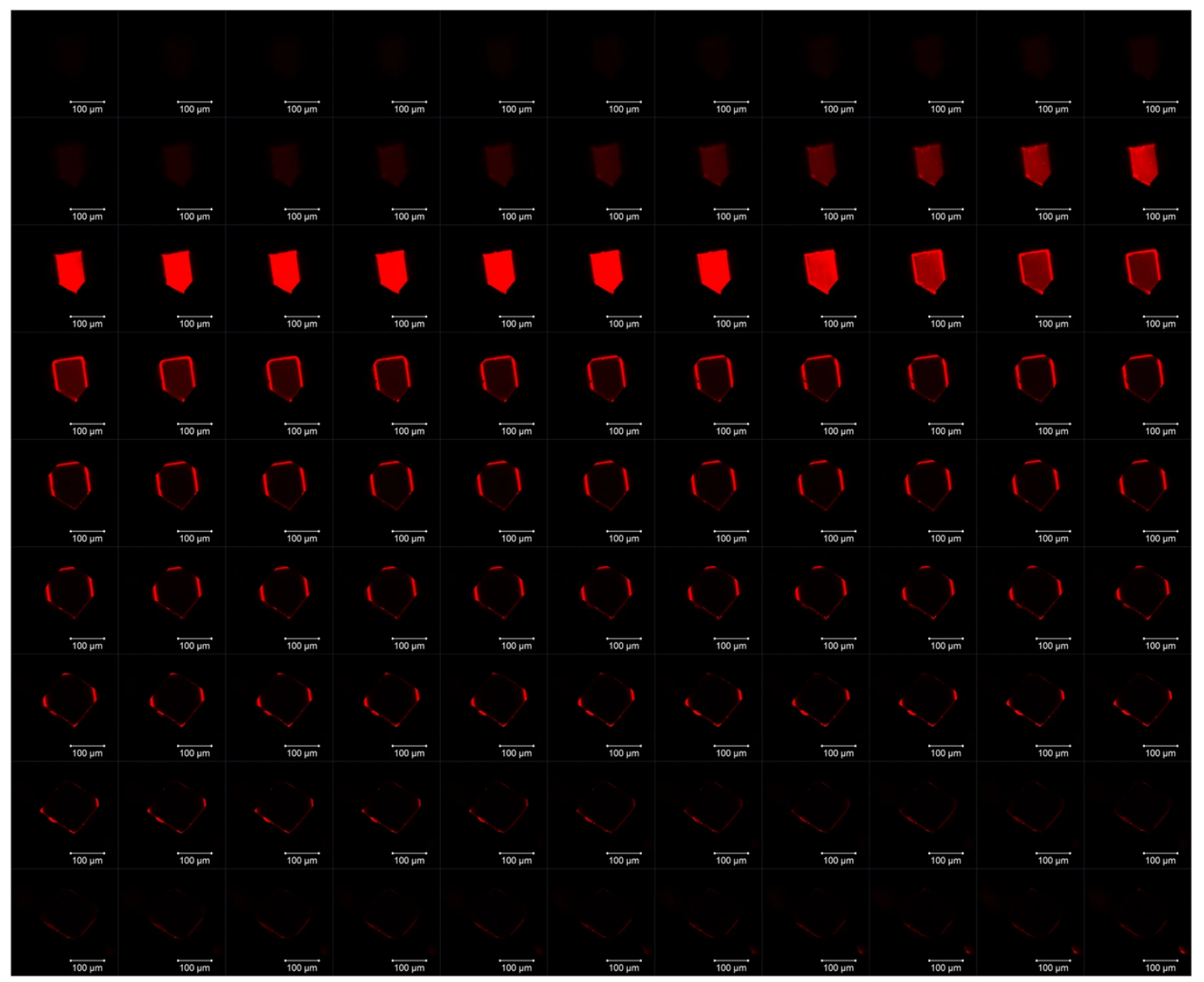

Figure 17. CLSM $z$-stack images of the R6G@In-BTB crystals, using a slicing distance of $1.25 \mu \mathrm{m}$. Excitation and emission wavelengths are $488 \mathrm{~nm}$ and $518 \mathrm{~nm}$, respectively. Adapted with permission from ref. 147. Copyright 2018 Nature Publishing Group.

The potential of CLSM is not only limited to the above experiments, and it has already been shown to be a powerful tool for imaging cells labelled with LG@MOF composites. For example, fluorescence micrographs of FL83B and HepG2 cells after being treated with luminescent Rs@nMOF-801 (Rs = resorufin) and R6G@UiO-67 (R6G = rhodamine 6G) exhibited intense red and yellow emission typically from Rs and R6G dyes trapped in the MOFs, which at the same time are incubated in the cells (Figure 18). ${ }^{79}$ 

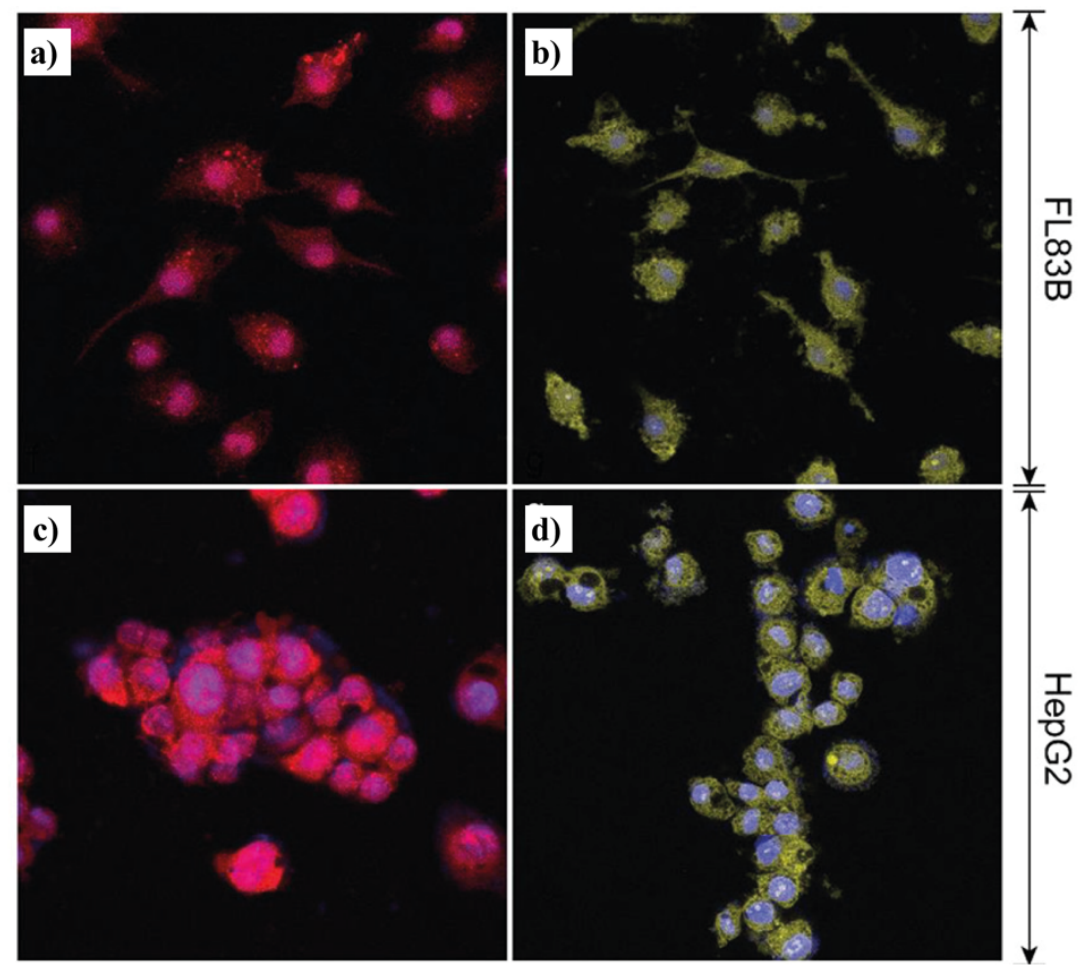

Figure 18. Fluorescence images of (a) FL83B using [Rs@nMOF-801]-GS, (b) FL83B using [R6G@nUiO-67]-GS, (c) HepG2 using [Rs@nMOF-801]-GS and (d) HepG2 using [R6G@nUiO-67]-GS. Adapted with permission from ref. 79. Copyright 2017 American Chemical Society.

\subsubsection{Scattering-type scanning near-field optical microscopy (s-SNOM) coupled with nano-Fourier transformed infrared spectroscopy (nanoFTIR)}

A scattering-type scanning near-field optical microscope (s-SNOM) coupled to a nanoFourier transformed infrared spectrophotometer (nanoFTIR) is a nanoscale local probe technique that simultaneously allows to image (measuring the height topography) and chemically characterize (by IR-active vibrational modes) multiple type of materials at a single crystal level. ${ }^{148}$ This system as illustrated in Figure 19a consists of an atomic force microscope (AFM) where a platinum-coated cantilever tip functions as a topographical and near-field optical probe simultaneously. When the sample is illuminated, the probe induces an evanescent near-field that act as a nanoscale light confiner, enhancer, and scatterer, paramount to obtain wavelength independent resolution. ${ }^{149}$ This technique, therefore, allows to collect AFM images while recording the nanoFTIR spectrum of a given material with a resolution down to $\sim 20 \mathrm{~nm}$. To the best of our knowledge, there is only one report using this technique to characterize LG@MOF materials. ${ }^{148}$ In this work, the authors demonstrate the guest encapsulation by combining s-SNOM/nanoFTIR with CLSM. As the s-SNOM/nano-FTIR is a surface 
technique, the authors scanned the surface of different LG@MOFs (RhB@ZIF-8, RhB@UiO66 and fluorescein@UiO-66) crystals before and after a thorough washing step. From those experiments, it can be deduced that before the washing step, it is possible to detect local vibrational modes associated with the guest molecules adsorbed on the MOF surface (Figure 19c, blue spectra). In contrast, after a thorough washing of the sample, there is a lack of vibrational modes associated with the guests, meaning no more guest molecules adhered on the MOF surface (Figure 19d, orange spectra). Then, the authors demonstrated by means of CLSM a homogenous distribution of fluorescein and RhB guests (with their characteristic green and red emission) over the MOF crystals in the washed samples. Therefore, it can be concluded that the homogeneous distribution of the guests together with the lack of signal derived from the nanoFTIR experiments unambiguously proved that the guests were effectively encapsulated into the MOF crystals. This study shows how complementary techniques and near-field analytics may be employed to verify the location of the guests in a single MOF crystal. 
a)

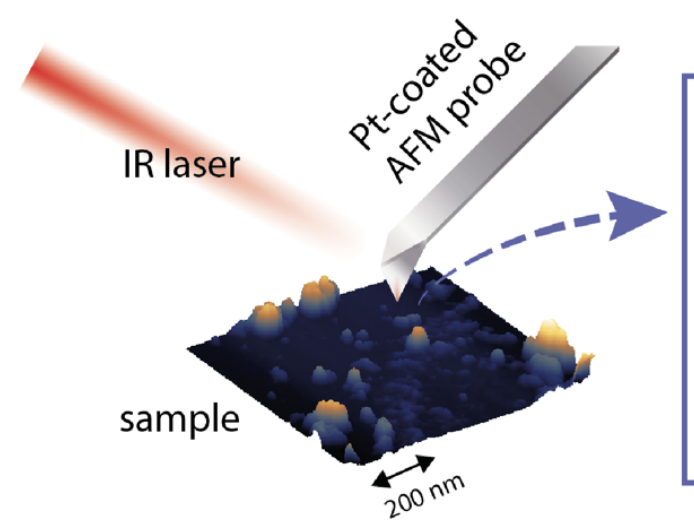

b) RhB@UiO-66

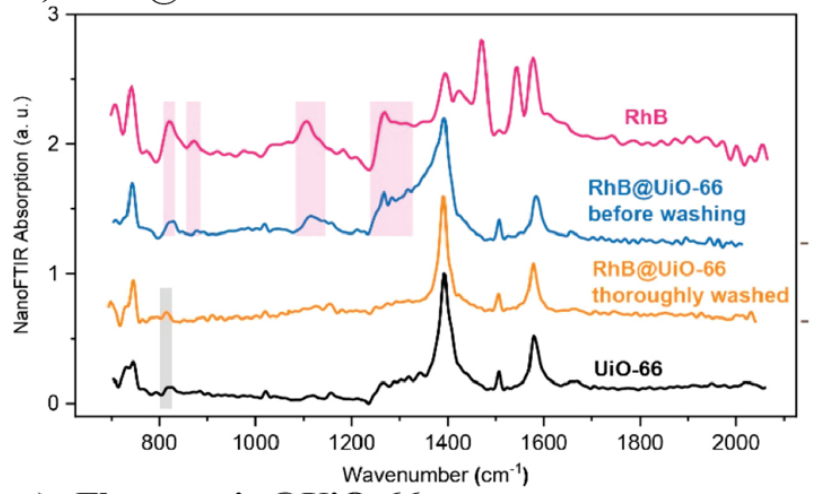

c) Fluorescein@UiO-66

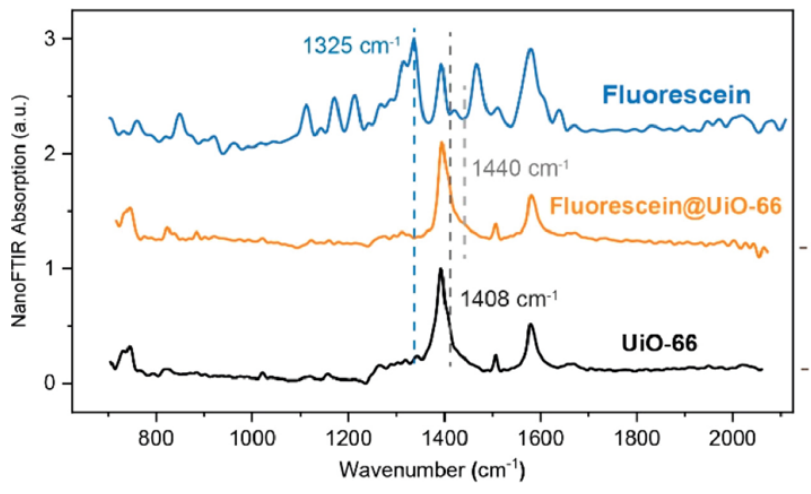

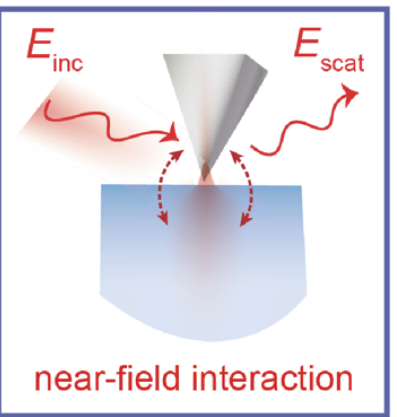

d)

e)
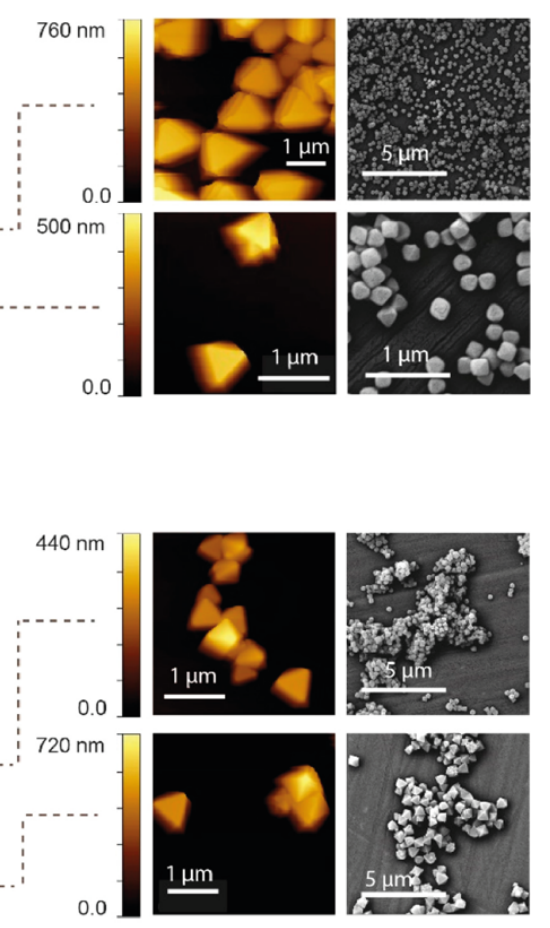

Figure 19. (a) Near-field optical spectroscopy (s-SNOM and nanoFTIR) using a nanofocus with a spot size of $\sim 20 \mathrm{~nm}$ illustrating the near-field interactions between the platinum-coated AFM probe and sample surface polarization upon illumination by a broadband infrared laser. $E_{\text {inc }}$ and $E_{\text {scat }}$ denote the incident and scattered light, respectively. (b) Near-field IR absorption spectra of RhB, UiO-66, as-synthesized RhB@UiO-66 composite before and after thorough washing. (c) NanoFTIR spectra of fluorescein, UiO-66 MOF and thoroughly washed fluorescein@UiO-66 composite. (d) AFM and (e) SEM images of UiO-66, RhB@UiO-66, and fluorescein@UiO-66 crystals. Adapted from ref. 148. Copyright 2020 American Chemical Society.

\subsubsection{Synchrotron and Neutron Based Techniques}

There are numerous synchrotron- and neutron-based characterization techniques that could be applied to probe a vast range of nanomaterials, soft matter, inorganic and organic 
compounds, including MOFs and composites. Broadly, these techniques can be divided into categories such as diffraction and crystallography, spectroscopy, imaging and microscopy. In essence, synchrotron and neutron facilities provide a high-flux source coupled with bespoke sample environments and advanced instrumentations to allow unconventional experiments to be performed under in situ and operando conditions. Our goal henceforth is not to be exhaustive, but to stimulate readers through several exemplars where the characterization of LG@MOF composite systems has benefited (or might benefit) from the application of stateof-the-art techniques at large science facilities.

X-ray absorption spectroscopy (XAS) ${ }^{150}$ can offer additional structural information in addition to diffraction techniques, since the latter is generally limited to the solution of an average structure. Particularly, XAS can reveal the local coordination within the inorganic cluster, and in doing so, shed light on the local structure and its deviation from the symmetry of the overall structure. ${ }^{151}$ In the context of LG@MOF systems, it is important to understand how the encapsulation of the fluorophores may distort the coordination environment surrounding the metal centers of the MOF host, and to locate the position of the guest species. Mustafa et al. ${ }^{152}$ reported a luminescent Eu@COK-16 system, where they employed a combination of XAS and XRD refinement techniques to determine the location of the exchanged $\mathrm{Eu}^{3+}$ ions within the $\mathrm{COK}-16$ host. The obtained spatial arrangement helps to elucidate the guest-host interaction facilitating intermolecular energy transfer processes, which underpin the luminescence behavior of this host-sensitized composite. In a related study concerning encapsulation of metal NCs within the Zn-based OX-1 MOF, Titov et al. ${ }^{153}$ demonstrated the implementation of synchrotron XAS techniques (XANES and EXAFS) to study the local structures around the Zn and Pd atoms in the Pd@OX-1 system. Interestingly, modelling of the XAS data revealed that the NCs in the composite are predominantly small clusters of Pd atoms, which are strongly interacting with the bdc linkers of OX-1. Zn K-edge measurements confirmed that the encapsulation of Pd guest, due to its small NCs, does not alter the framework structure around the $\mathrm{Zn}$ centers of the OX-1 host.

Synchrotron-radiation infrared (SR-IR) spectroscopy presents many advantageous compared with the conventional benchtop FTIR instrument because it is broadband and has a very high flux, thereby ideal for acquiring high-resolution broadband vibrational spectra of MOF materials, especially to probe low-frequency phonons in the far-IR regime (terahertz frequencies $\lesssim 10 \mathrm{THz}$ ). ${ }^{154}$ These $\mathrm{THz}$ modes are important as they are underscoring the elasticity, structural stability, guest-host dynamics, phase transformation and functions of all framework materials. ${ }^{155-159}$ When combined with inelastic neutron scattering (INS), all 
vibrational modes from $0-4,000 \mathrm{~cm}^{-1}$ can be measured since there is no optical selection rules when employing neutron as a probe. ${ }^{160}$ However, detailed assignment of the vibrational modes will often require further insights derived from DFT simulations as exemplified by these studies. ${ }^{154,159,161}$ Particularly, the theoretical modelling of the host-guest interactions of a bulky fluorophore with a MOF host is not trivial, this is a challenging task for DFT as demonstrated in this recent work on the fluorescein@ZIF-8 system. ${ }^{83}$ Finally, it is worth emphasizing that the high spatial and temporal resolution afforded by the synchrotron and neutron sources permit the in situ/operando experiments to investigate host-guest interactions and to gain insights into basic mechanisms subject to different physical and chemical stimuli, such as UV irradiation, ${ }^{162}$ temperature, ${ }^{163}$ pressure, ${ }^{164}$ and various kinds of guest molecule. ${ }^{165-170}$

In terms of the 3-D imaging of MOF crystals using synchrotron irradiation, recently, techniques such as the full-field transmission X-ray microscopy (TXM) nanotomography has been demonstrated by Mayorga-Gonzalez et al. ${ }^{171}$ In this study, a single crystal of MIL-47(V) with its smallest cross-section of $\sim 10 \mu \mathrm{m}$ has been reconstructed to yield a 3-D map revealing the internal microscopic porosity for subsequent quantitative analysis. Surprisingly, the results derived from TXM show that the actual porosity level was only 2-3 vol.\%, but this value has been greatly overestimated by $\mathrm{Hg}$-intrusion by a factor of about ten times. Related to the above is scanning tunnelling X-ray microscopy (STXM), a spectro-microscopy technique resembling SEM/TEM imaging, but enables one to perform nanoscale chemical imaging using X-ray photons. ${ }^{172}$ Another 3-D imaging technique demonstrated was full-field tomographic XAS ${ }^{173}$, where a defect-engineered HKUST-1 single crystal $(\sim 50 \mu \mathrm{m})$ was studied to visualize its spatio-chemical heterogeneities and identify secondary phases at a resolution of $\sim 2 \mu \mathrm{m}$. It will be exciting to explore the use of synchrotron 3-D imaging tools to interrogate LG@MOF systems; for example, to visualize micro/nanostructural defects and to quantify chemical heterogeneities of NCs@MOF and QDs@MOF composite crystals, as well as to interrogate the sample performance under in situ/operando conditions.

\section{GUEST-HOST PHENOMENA - EXCITED STATE EVENTS}

Excited state proton transfer (ESPT), charge transfer (CT) and Förster energy transfer (FRET) phenomena are key events in many natural (e.g., photosynthesis, biological processes) and mimicked artificial systems (e.g., solar cells, OLEDs, photocatalysis), being the keystone of many of the modern advanced photonic and optoelectronic technologies. ${ }^{174-177}$ Thus, a proper control and understanding of those excited state mechanisms is paramount for a better 
development of advanced functional materials, and consequently, for the fabrication of the next generation of optoelectronic and photonic devices. Over the past two decades, the steady-state and ultrafast photodynamics of the ESPT, CT and FRET processes have been well documented in different guest-host composites, such as guest encapsulated into different silica-based mesoporous (SBMs) systems. ${ }^{92,178,179}$ However, to assure a sustainable world-wide growth, there is an urgent need of developing more efficient materials that will bring new practical and cost-effective solutions to address the global challenges, such as renewable energy generation and its efficient use, among others. To this end, the tunable physicochemical properties of MOFs have turned them into one of the most promising materials to explore, improve and exploit these excited-state phenomena. In the following sections, we will describe the basic theory behind those photophysical events, including some of the most representative examples in the field of MOFs.

\subsection{Förster Resonance Energy Transfer (FRET)}

FRET is a non-radiative physical event in which a photoexcited fluorophore (donor) will transfer the energy to an acceptor pair. Several criteria must be satisfied in order to harvest the energy efficiently: (i) Firstly, the distance between the donor and acceptor entities must be sufficiently close (typically 10-100 $\AA$ ), as the efficiency of the process $(\eta)$ decreases with the sixth power of the distance:

$$
\eta=\frac{R_{0}^{6}}{R_{0}^{6}+R^{6}}
$$

where $R_{0}$ stands for the Förster radius (distance at which the energy transfer is $50 \%$ efficient), while $R$ is the donor-acceptor distance. (ii) Secondly, there must exist an overlap between the emission spectrum of the donor species and the absorption spectrum of the acceptor. (iii) Thirdly, the orientation of the dipole momentum of the donor and acceptor entities must be sufficiently good (typically should be parallel) to allow a dipole-dipole coupling as:

$$
\begin{aligned}
& R_{0}=0.211\left[\kappa^{2} n^{-4} \Phi J(\lambda)\right]^{1 / 6} \\
& J(\lambda)=\int_{0}^{\infty} F_{\mathrm{D}}(\lambda) \varepsilon_{\mathrm{A}}(\lambda) \lambda^{4} d \lambda
\end{aligned}
$$

where $\kappa$ is a factor describing the transition dipoles in space (generally assumed as $2 / 3$ ), $n$ is the refractive index of the medium, $\Phi$ is the quantum yield of the donor in the absence of the acceptor, and $J(\lambda)$ is the overlap integral, which at the same time depends on the $4^{\text {th }}$ power of 
the wavelength $\left(\lambda^{4}\right)$, the emission of the donor normalized to an area of $1\left(F_{\mathrm{D}}(\lambda)\right)$, and the extinction coefficient of the acceptor at a given wavelength $\left(\varepsilon_{\mathrm{A}}(\lambda)\right)$. Albeit not a critical requirement, it is desirable that both, the donor and acceptor fluorophores exhibit a high fluorescence quantum yield.

Based on the foregoing statements, it is clear why MOFs are an excellent platform to trigger the FRET mechanism. On the one hand, their accessible pore cavities can allow the encapsulation of a vast range of fluorophores, which will shorten the donor-acceptor distances. On the other hand, the almost infinite combination of luminescent MOFs (LMOFs) and types of fluorophore guest (Section 3) provides a unique opportunity for enhancing the spectral overlap through a judicious selection of the donor and acceptor entities. Moreover, the crystalline structure of MOFs allows one to predict the position of the donor and acceptor fluorophores, offering a promising pathway for controlling directional FRET, which can occur from the MOF to the guest or vice versa. ${ }^{96,180-182}$

Some of the most powerful tools to unravel FRET processes are based on steady-state and time-resolved emission techniques, such as fluorometer, time-correlated single photon counting (TCSPC), or femtosecond (fs) up-conversion techniques. Typically, one of the first observation in a FRET process is the quenching of the emission intensity of the donor system. Although this is not an indisputable scientific evidence (as other excited state processes may produce the same phenomenon), it could be an indication of a FRET mechanism. Indeed, a very common experiment is to load the MOF with an increasing amount of guest dyes to incrementally show the quenching of the emission intensity of donor, and the enhancement in the emission intensity of the acceptor. Some of these findings are summarized in Figure 20, where different amounts of coumarin, RhB, or DCM fluorophores were encapsulated into different MOFs, showing incremental quenching of the MOF emission when subject to a greater concentration of guest. ${ }^{86-88}$ 

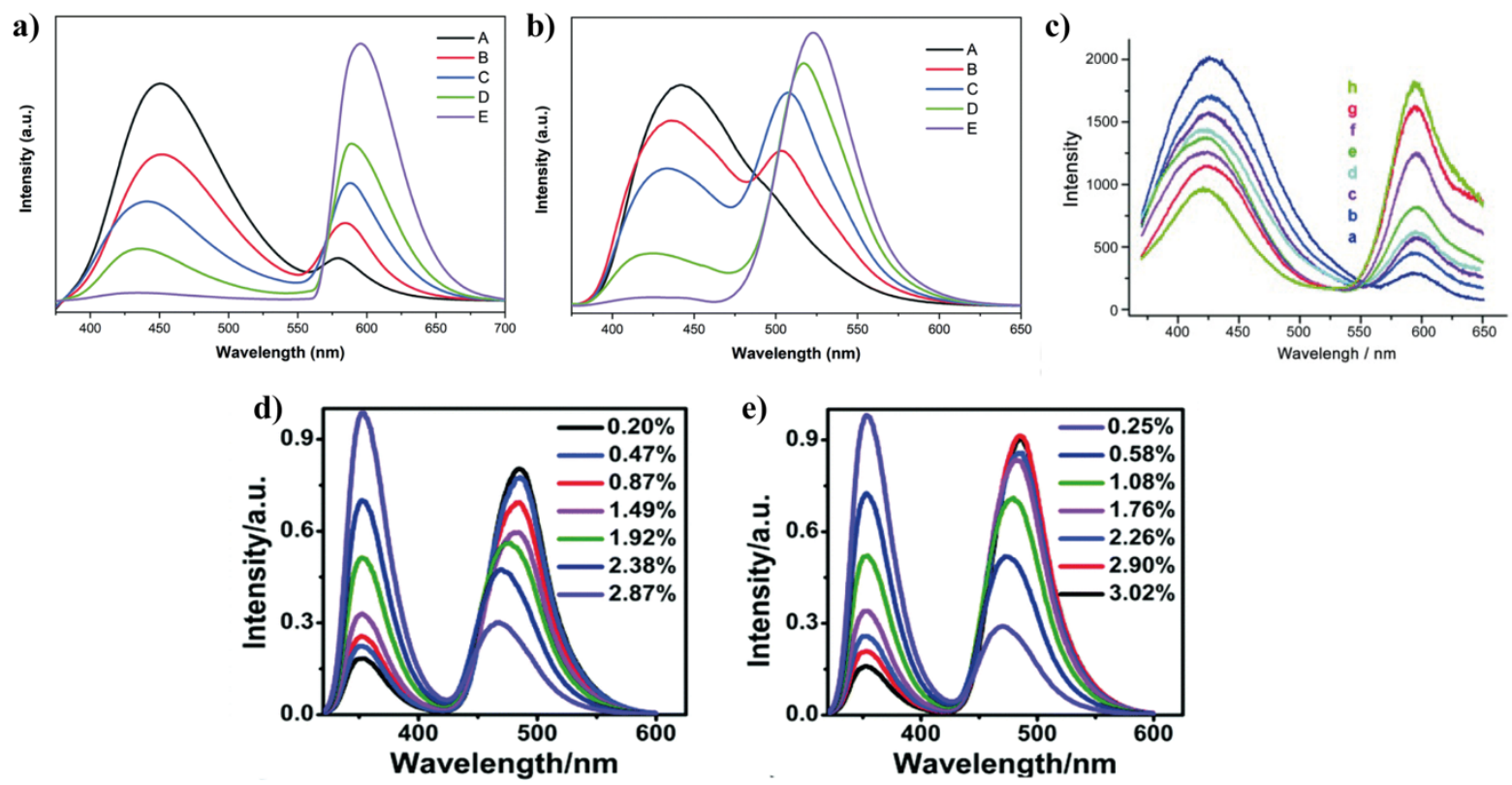

Figure 20. (a-b) Emission spectra of A) $1 \supset \mathrm{DCM}$ and B) $1 \supset \mathrm{C} 6$ with different concentration of dyes: A: $1 \times 10^{-6} \mathrm{mmol} \mathrm{L}^{-1}$; B: $5 \times 10^{-6} \mathrm{mmol} \mathrm{L}^{-1}$; $\mathrm{C}: 1 \times 10^{-5} \mathrm{mmol} \mathrm{L}^{-1}$; D: $5 \times 10^{-5} \mathrm{mmol}$ $\mathrm{L}^{-1}$; and $\mathrm{E}: 1 \times 10^{-4} \mathrm{mmol} \mathrm{L}{ }^{-1}$. Adapted with permission from ref. 88. Copyright 2018 Royal Society of Chemistry. (c) Emission spectra of RhB@CZJ-3 with different concentration of RhB: $a=0.02$ wt. $\%, b=0.04$ wt. $\%, c=0.06$ wt. $\%, d=0.08$ wt. $\%, e=0.1$ wt. $\%, f=0.12$ wt. $\%$, $\mathrm{g}=0.16$ wt. $\%$, and $\mathrm{h}=0.19 \mathrm{wt} . \%$. Adapted with permission from ref. 86. Copyright $2014 \mathrm{John}$ Wiley \& Sons. (d-e) Emission spectra of coumarin/M-BTB MOFs with different levels of coumarin loading. Adapted with permission from ref. 87. Copyright 2017 Royal Society of Chemistry.

A more reliable proof can be obtained by measuring the excitation spectrum, selecting the emission (observation) wavelength in a region where the emission of the acceptor can be detected, but where there is no emission signal from the donor. If there is a FRET process, then the excitation spectrum must show the feature of the spectrum (usually comparable to the absorption one) of the donor counterpart. For example, the excitation spectra of a series of lanthanides in the bio-MOF-1 (Tb@bio-MOF-1, Eu@bio-MOF-1 and Tb/Eu@ bio-MOF-1) display a band in the range of $230-400 \mathrm{~nm}$, which matches the absorption of the MOF linker (4,4-biphenyldicarboxylic acid), reflecting the sensitization of the lanthanides by the latter. ${ }^{108}$ A more visual example is reported for the energy transfer process occurring from a Zr-NDC MOF to different organic fluorophores (coumarin 153 (C153), nile red (NR) and DCM). In those materials, the excitation spectra - recorded in spectral regions where the MOF is not emitting but there still some emission of the dyes - clearly show the vibrational bands of the 2,6-naphthalenedicarboxylate (NDC) linkers of the MOF, thereby unequivocally proving the occurrence of a FRET process from the MOF to the guests. ${ }^{85}$ Similarly, the FRET process from 
a Hf-MOL (metal organic layer) to the coumarin 343 (C343) was confirmed by monitoring the excitation spectrum at $475 \mathrm{~nm}$ (emission of C343), which exhibits two peaks corresponding to the direct excitation of C343 (445 nm) and the linker of the MOF $(290 \mathrm{~nm}) .{ }^{87}$

Time-resolved experiments can help to decipher the photodynamics of the FRET mechanisms alongside the lifetime of donor and acceptor entities. Here it is generally important to distinguish between the emission regions of the donor and the acceptor. In the emission region of the donor, the lifetime $\left(\tau_{\mathrm{D}}\right)$ should decrease as the emission will be quenched. Additionally, if the time resolution of the system is good enough, one can observe an additional shorter time component with a positive amplitude that may correspond to the time of the FRET phenomenon. On the other hand, the lifetime of the acceptor (recorded at longer wavelengths) may depend on the surrounding environment, as changes in the polarity, acidity/basicity, or even the confinement effect will alter this value. In addition to that, and again, if the time resolution of the instrument is adequate, it is possible to observe a new shorter time component, comparable to that observed in the region of the donor, but now with a negative amplitude. This negative amplitude (rise component) reflects the population of the excited state of the acceptor moiety, and therefore, this will also correspond to the duration of the FRET phenomenon.

Although it is primordial to unveil the ultrafast dynamics of FRET processes in order to enhance the efficiency of this mechanism, the number of studies focusing on the photodynamics of LG@MOF undergoing FRET is much lower than for other systems such as Guest@SBMs. Still some interesting examples can be found in the literature. ${ }^{85,118,183,184}$ For example, the encapsulation of rhodamine 6G (Rh6G) into the MOF $\left[\mathrm{Zn}_{6} \mathrm{~L}_{4}\left(\mathrm{Me}_{2} \mathrm{NH}_{2}{ }^{+}\right)_{4} \cdot 3 \mathrm{H}_{2} \mathrm{O}\right]$ yields a material showing a highly efficient energy transfer (98.8\%). ${ }^{183}$ In this system, the average lifetime of the MOF (donor) in the absence of the Rh6G (acceptor) is $7.63 \mathrm{~ns}$, while increasing amounts of Rh6G in the pores of the MOF decreased the lifetime to $0.16 \mathrm{~ns} .{ }^{183}$ Similarly, the energy transfer from carbon dots (CD) to curcumin (CCM) dye, both entrapped into ZIF-8 MOF, was demonstrated by the quenching of the $\tau_{\mathrm{D}}(\mathrm{CD})$ from $5.77 \mathrm{~ns}$ in the absence of CCM to $3.03 \mathrm{~ns}$ in the presence of the latter. ${ }^{118}$ Another analogous example is given by the decrease in the lifetime of a BODIPY (donor) encapsulated in the nPCN-222 MOF (acceptor). ${ }^{184}$ A detailed ultrafast photophysical characterization of a FRET from a Zr-NDC MOF to different guest dyes was reported by Douhal, et al. ${ }^{85}$ In this work (mentioned also above), the authors encapsulated 3 different dyes (C153, NR and DCM) in the pores of $\mathrm{Zr}$ NDC, whose emission may originate from linker monomers or excimers. It was demonstrated that for C153@Zr-NDC, the lifetime of the monomers was quenched, however, for NR@Zr- 
NDC and DCM@Zr-NDC the lifetime corresponding to the excimeric species was the one who suffer a decline. This result indicates that in the first case, the transfer of energy takes place from the monomers of Zr-NDC to the C153, while in the other systems it occurs from the excimer of the MOF to the guest dyes (Figure 21). Moreover, the authors also detected a shorter component (hundreds of ps) with positive (donor emission region) and negative (acceptor emission region) amplitudes, attributed to the time of the FRET process (Figure 21). ${ }^{85}$ Many of these LG@MOF composites undergoing FRET have a great potential for many photonic applications, such as luminescence sensing or solid-state lighting (see Section 6).
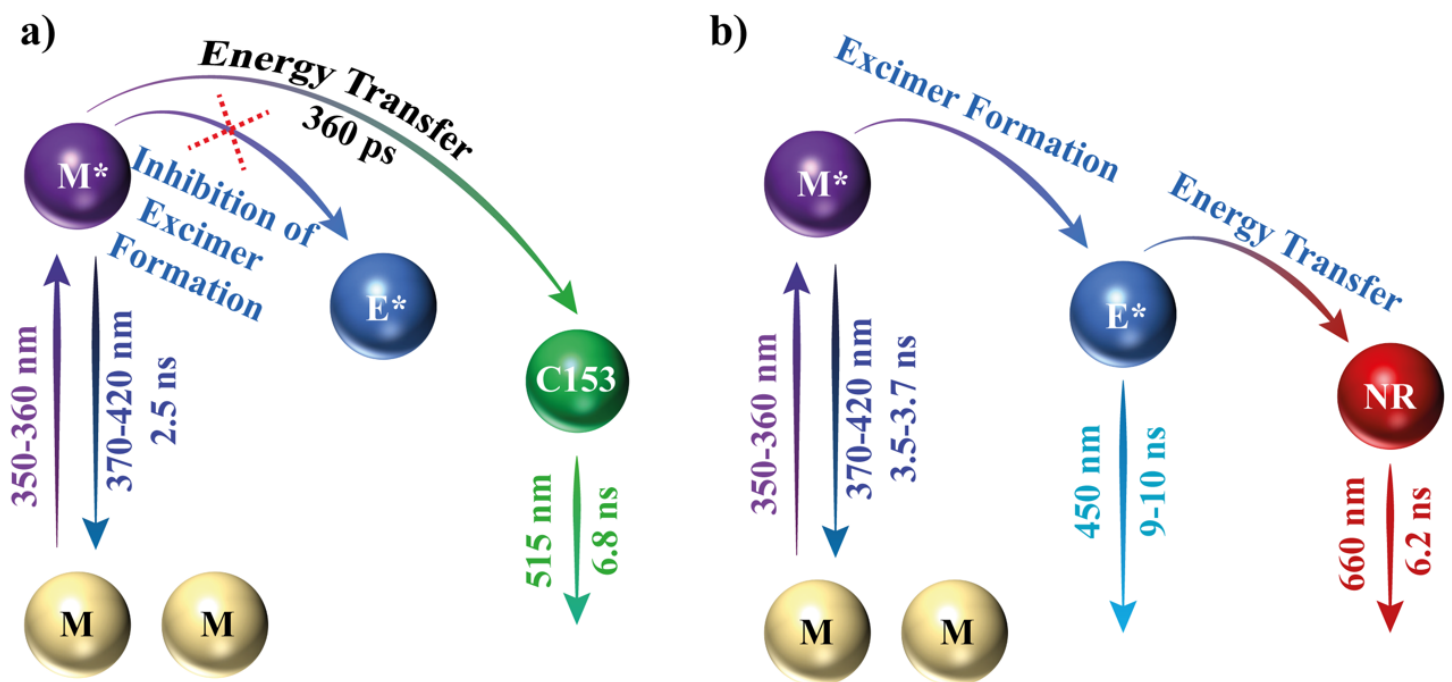

Figure 21. Schematic representation of the underlying photophysical processes after the photoexcitation of (a) 153@Zr-NDC and (b) NR@Zr-NDC MOF composites. In scheme (a), the encapsulation of $\mathrm{C} 153$ inhibits the formation of the excimeric species, and the energy transfer occurs from the monomers (denoted as M). However, in the case of NR@Zr-NDC (scheme b), firstly the excimers are formed and then those transfer their energy to the NR dye. Adapted with permission from ref. 85. Copyright 2015 Royal Society of Chemistry.

\subsection{Photoinduced Charge Transfer (PCT)}

Photoinduced charge transfer (PCT) is a photophysical process that involves the transfer of charge (or an electron) from a photoexcited electron donor to an electron acceptor system. This process can be either intramolecular, when the electron donor and acceptor belong to the same molecule, or intermolecular in the case when the electron donor and acceptor are different molecules. The PCT is one of the most important photophysical events in nature as it is the driving force of essential biological phenomena, such as the photosynthesis or the human vision. ${ }^{185}$ In addition to that, researches have mimicked these processes to develop some of the most important advanced technologies, such as optoelectronic devices (solar cells, OLEDs), 
photocatalysts, or chemical sensors. ${ }^{174-177,186,187}$ Hence, the control and manipulation of PCT through the design, fabrication and characterization of novel materials are paramount to enhance the efficiency of such technologies. The synthetic flexibility of MOFs coupled with their porosity, make these materials ideal scaffolds to explore the PCT phenomenon. One of the simplest and most exploited method to induce PCT in MOFs is through the design of redoxactive and/or electron-rich, or electron-deficient organic linkers; this topic has been summarized in a recent review by Deria and coworkers. ${ }^{188}$

However, and more in line with the current review focusing on LG@MOF systems, another interesting approach is the encapsulation of PCT-active molecules in the pores of the MOF. In one of the first reports in this subject, Allendorf et al., proposed a possible PCT process from the linker (1,3,5-benzenetribenzoate) of the MOF-177 to the encapsulated [6,6]phenyl-C61-butyric acid methyl ester (PCBM) molecule. ${ }^{189}$ Although it was not possible to prove it experimentally, the authors have reasoned that this PCT exists based on the emission quenching observed for the MOF and on theoretical calculations, which predicts the possibility for a charge transfer to occur. ${ }^{189}$ Thereafter, a variety of PCT-active compounds have been encapsulated in the pores of multiple MOFs. ${ }^{84,188,190,191}$ Indeed, an interesting strategy to detect electron-rich or electron-deficient analytes (e.g., explosives and pollutants) is based on the employment of active linkers to provoke a PCT reaction from or to the encapsulated guests. These host-guest interactions will alter the photophysical properties of the MOFs by quenching, enhancing, or shifting (change in color) of their emissions. For instance, there exist many examples of luminescent MOFs which are able to detect nitroaromatic explosive-like molecules due to their emission quenching produced by a PCT from the MOF to those electrondeficient molecules. ${ }^{192-195}$ Another intriguing example has been reported by Maji and coworkers, where different electron-donating aromatic amines were encapsulated in the pores of a blue light emitting Zn-based MOF ([Zn(ndc)(o-phen)] DMF) ${ }^{84}$ The strong CT interactions between the amines and the 1,10-phenanthroline (o-phen) linker produce a large shift in the emission, resulting in multicolor fluorescence, which is dependent on the aromatic amine (Figure 22); the results show the outstanding ability of this MOF to detect specific analytes that can be easily observed by the naked eye. ${ }^{84}$ 


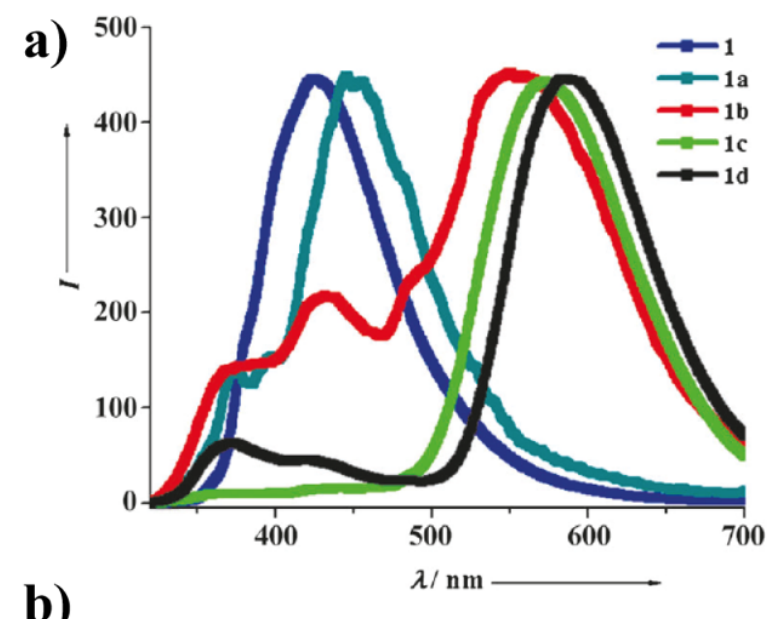

b)

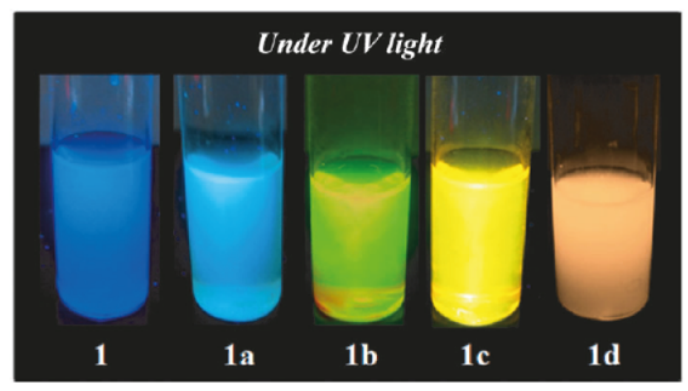

Figure 22. (a) Emission spectra of $[\mathrm{Zn}(\mathrm{ndc})(\mathrm{o}-\mathrm{phen})] \cdot \mathrm{DMF}$ (1) MOF before and after the inclusion of aniline (1a), N-methyl aniline (1b), $N, N^{\prime}$-dimethyl aniline (1c), and $N, N^{\prime}$ dimethyl-p-toluidine (1d), upon excitation at $\lambda=330 \mathrm{~nm}$. (b) Photographs of compounds 1 and 1a-1d after being dispersed in methanol and under UV light irradiation. Reproduced with permission from ref. 84. Copyright 2014 John Wiley \& Sons.

In addition to the aforementioned exemplars, another strategy is to encapsulate fluorophores undergoing photoinduced intramolecular charge transfer (ICT). Time-resolved transient absorption and emission techniques have proven to be a very powerful tool to unveil these PCT processes. ${ }^{190,191}$ For instance, it has been possible to interrogate in real time the ICT phenomenon taking place in the fluorophore NR dye, when it is interacting with a series of 2-D MOFs. ${ }^{190,191}$ Depending on the 2-D MOF, this ICT process in the NR dye occurred in times as short as 1 ps or $425 \mathrm{fs}$. Similar to the previous example, the authors took advantage of this PCT process to characterize the interactions between the electron-rich aniline and dimethylaniline aromatic molecules and the NR dye. This investigation was carried out by exposing the LG@MOF to vapors of the aromatic amines and by functionalizing the MOF with aniline, showing in both cases a strong CT interaction from the analytes to the NR dye. In fact, the authors reported an ultrafast electron transfer from aniline to NR molecules happening over a short time span of 17 ps. ${ }^{190,191}$ This is therefore another interesting mechanism for the detection of electron-rich or electron-deficient toxic vapors by leveraging LG@MOF systems. 


\section{3. $\quad$ Excited-State Proton Transfer (ESPT)}

The excited-state proton transfer (ESPT) is a process in which a photoexcited species transfers a proton from a donor group (typically $-\mathrm{OH},-\mathrm{NH},-\mathrm{SH}$ ) to an acceptor (e.g., $\mathrm{C}=\mathrm{O},-$ $\mathrm{N}=$ ). This is a photoinduced process, as the irradiation with photons induces a change in the electronic distribution of the molecule, affecting the acidity/basicity of the proton donor and acceptor groups, which at the end controls the efficiency of the process. Similar to the PCT, the ESPT process can be either intramolecular (ESIPT), when the proton donor and acceptor groups are distributed within the same molecule, or intermolecular, when the donor and acceptor groups belong to different molecules. ${ }^{196}$ The ESPT phenomenon produces, therefore, a tautomerism between the enol and keto, or the conversion to ionic species (anion or zwitterion), which exhibit a different optical and photodynamical behavior. In the case of an enol-keto tautomer, the absorption spectrum remains unchanged, as the proton transfer occurs after photoirradiation. However, the emission spectra will be very different as a function of the emitting species. The emission of the enol tautomer is more energetic, while the emission of the keto species appears at larger wavelengths, the Stokes shift being much higher. The possibility of tuning a dual-color emission from the same molecule, alongside with the fact that the proton transfer reaction is one of the most common phenomena in several chemical and biological processes, has made ESPT one of the most well characterized mechanisms. Since the pioneering works of Weller, ${ }^{197,198}$ Weber, ${ }^{199}$ and Förster ${ }^{200}$ in the 1950 s, this subject has been boosted through the design, synthesis and detailed characterization of molecules, materials, and composites that undergo ESPT. ${ }^{92}$

Surprisingly, the ESPT mechanism has not yet been extensively exploited in the MOF systems, and just a handful of examples hitherto have been reported. ${ }^{201-209}$ Most of them, are based on MOFs where the linker can undergo an ESPT, such as 2,5-dihydroxyterephthalic acid (DHT). For instance, a number of different metal-based MOFs ( $\mathrm{Mg}, \mathrm{Cd}, \mathrm{Y})$ have been reported whose photophysical properties are governed by an ESIPT reaction within the DHT linker. ${ }^{203-}$ 205,207,209 As the efficiency of the ESIPT process, and therefore the enol-keto tautomerism, will be directly influenced by the surrounding environment, these MOF materials exhibit excellent capabilities toward the detection of many chemical compounds such as different organic solvents, ${ }^{202-205}$ water, ${ }^{207}$ cations, ${ }^{203,207}$ anions, ${ }^{204,207}$ and vapors of different amines. ${ }^{209}$ Similarly, another microporous Zn-MOF where the linker, 5-(2-(5-fluoro-2-hydroxyphenyl)4,5-bis(4-fluorophenyl)-1H-imidazol-1-yl)isophthalic acid, experiences an ESIPT reaction, 
was demonstrated to be an outstanding candidate for the rapid detection of water owing to the enol-keto phototautomerism. ${ }^{206}$ Another very interesting example of the use of ESIPT linkers in the construction of MOFs is the one reported by Uribe-Romo and coworkers. ${ }^{208}$ The authors have designed ESIPT linkers with the capacity of emitting blue, green and red light, which were then utilized in different percentages to construct a Zr-based MOF (Figure 23). Interestingly, the MOF retained the emission of the linkers, exhibiting multicolor and highquality white light emission.

Further to the very few reports we exemplified above, and to the best of our knowledge, there are no examples of ESPT molecules encapsulated in MOFs, so this area presents a hugely unexplored field in the context of LG@MOF designs. Some of the big questions that need to be investigated on this regard will be as follows. (1) Would it be possible to encapsulate ESIPT molecules within the MOFs while retaining the intramolecular proton transfer reaction? (2) Would it be possible to manipulate, control, and tune intermolecular ESPT reactions between a proton-transfer guest and a MOF containing functionalized linkers (e.g., with $-\mathrm{CN},-\mathrm{SH}$ or $\mathrm{NH}_{2}$ )? (3) Would it be feasible to leverage the novel ESPT@MOF materials to develop luminescent sensors, or to yield other photonic devices?

a)

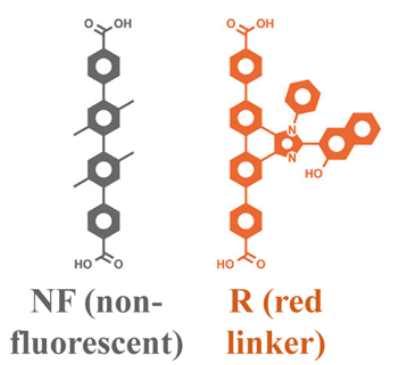

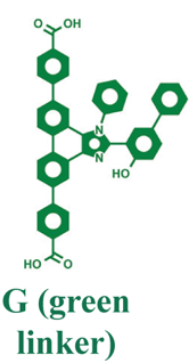

c)

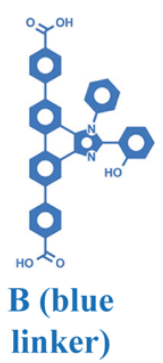

b)

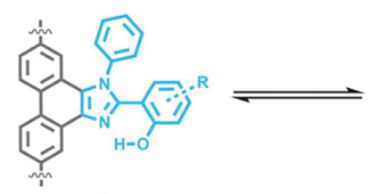

Enol form

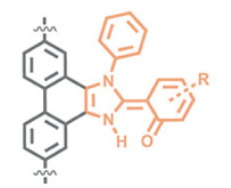

Keto form
$\mathrm{Zr}_{6} \mathrm{O}_{4}(\mathrm{OH})_{4}\left[\left(\mathrm{R}_{x} \mathrm{G}_{1-2 x} \mathrm{~B}_{x}\right)_{0.01} \mathrm{NF}_{0.99}\right]_{6}$

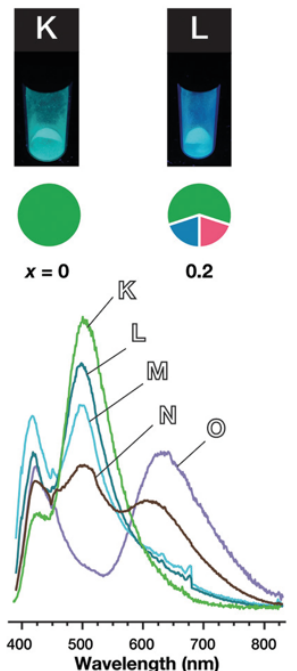

Figure 23. (a) Multicolor emitting organic linkers exhibiting ESIPT. (b) Schematic representation of the enol and keto phototautomerism. (c) Photographs taken under UV light irradiation ( $\lambda_{\mathrm{ex}}=365 \mathrm{~nm}$, top), emission spectra (bottom left) and CIE coordinates (bottom right) of MOFs having different ratio of R, G, and B ESIPT linkers. Adapted with permission from ref. 208. Copyright 2019 American Chemical Society. 


\subsection{Confinement Effects, Weak Interactions, and Aggregates Formation}

The encapsulation of LGs in the pores of the MOFs may induce alterations of their structure and photophysical properties. On the one side, it is well-known that many organic fluorophores are sensitive to the nature of the surrounding environments, such as polarity or acidity/basicity, usually leading to bathochromic (red) or hypsochromic (blue) shifts depending on the characteristics of the guest materials. A typical example of these type of organic dyes are the coumarins. For instance, while the emission maximum of the coumarin 460 (C460) in methanol (or ethanol) is at $\sim 450 \mathrm{~nm},{ }^{210}$ it has been demonstrated that it could shift to $464 \mathrm{~nm}$ or $430 \mathrm{~nm}$ upon its encapsulation into $\mathrm{Eu}_{0.05} \mathrm{~Tb}_{0.95} \mathrm{BPT}$ or Tb-TATAB MOFs, respectively. ${ }^{76,77}$ Similarly, the emission maximum of coumarin 343 (C343) slightly shifts from $482 \mathrm{~nm}$ in methanol, ${ }^{210}$ to $475 \mathrm{~nm}$ upon its encapsulation in a Hf-MOL. ${ }^{87}$ Highly distinct is the red-shift of $30 \mathrm{~nm}$ (1250 $\mathrm{cm}^{-1}$ ) observed in the emission spectrum of $\mathrm{C} 153$ upon its encapsulation in a Zr-NDC MOF. ${ }^{85}$

On the other hand, the restriction imposed on the fluorophores by their entrapment or caging into the MOFs - known as the confinement effect - generally decreases or even suppresses the non-radiative processes (e.g., molecular motions, that deactivate the fluorophore in the form of heat), turning into an augmentation of the emission quantum yield and lifetime. For instance, the encapsulation of a BODIPY dye, PM605, in the restricting pores of ZIF-8 decreases the non-radiative processes undergoing in the photoexcited PM605, resulting in an increase of the average lifetime from $6.8 \mathrm{~ns}$ (acetonitrile solution) to $7.5 \mathrm{~ns} .^{211}$ Similarly, the confinement of the metal complex ZnQ in the OX-1 MOF structure, enhances the luminescence quantum yield (from $\Phi=35 \%$ in DMF suspension to $44 \%$ in the MOF) and extends the lifetime of the fluorophore. ${ }^{35}$ Another typical example of confinement effect has been described for the metal-organic complex Ru(II)tris(2,2'-bipyridine) (Rubpy) after its encapsulation into different MOFs. ${ }^{212-214}$ In all these examples, the caging of the Rubpy by the MOF host prevents its expansion, increasing the energy barrier to populate the ${ }^{3} \mathrm{LF}$ state, suppressing the major nonradiative deactivation pathway, and therefore, resulting in a rise of the emission lifetime. ${ }^{212-214}$ This confinement effect has also been reported for $\mathrm{Cu}$ nanoclusters incarcerated into ZIF-8, where it was observed a 20 -fold increase of the luminescence quantum yield (from $0.5 \%$ to $11 \%$ ) accompanied by a substantial rise of emission lifetime (from 1.3 to $11 \mu \mathrm{s}$ ). ${ }^{132}$ Surprisingly, the role of the confinement effect has been neglected in many other reports, where the increment of the emission lifetime of the encapsulated guests is solely attributed to an energy transfer process, while it was not considered as a possibility that the guest entrapment 
or caging phenomenon may contribute even more importantly to the rise of the emission lifetime value..$^{98,138,139,215}$

In addition to the aforementioned effects, the encapsulation of increasing concentrations of LGs into MOFs may give rise to the formation of guest aggregates (H- or J-aggregates), which generally shift the emission to the reddest part of the spectral region (owing to the presence of J-aggregates), and could decrease the emission intensity if the concentration becomes too high. Therefore, the potential applicability of LG@MOF materials in photonic technologies will strongly depend on a proper control and careful manipulation of the amount of encapsulated guest loading within the MOF host. Excessive amount of guest will generally lead to the formation of aggregates, while insufficient concentration of LGs will prevent a high luminescence intensity. Thus, for certain purposes, it is necessary to prepare different LG@MOF composites, with increasing amounts of LGs to estimate the optimal concentration, where the luminescence intensity is the highest while the concentration of aggregates is minimal but still present.

Even though the observation of a red-shift in the emission spectrum with an increasing amount of organic dyes confined in MOFs is a well-known observation in many studies, ${ }^{74,78,136}$ in general, there is a lack of information or the phenomenon is vaguely explained. ${ }^{74,75,78,136,138,139,142}$ In fact, some reports attribute this effect to aggregation, autoabsorption (reabsorption) or intermolecular interactions favored by the incorporation of fluorophore dyes in the MOF host. ${ }^{75,138,139,142}$ Although all these processes may induce a redshift of the emission spectrum, there are some experiments that could assist in unravelling the formation of the J- and H-aggregates. Firstly, it is well known that the $\mathrm{S}_{0} \rightarrow \mathrm{S}_{1}$ transition of Haggregates is more energetic than the monomers, while that of the J-aggregates is less energetic. This means that the absorption spectrum will be broadened upon the formation of the aggregated species, and a proper deconvolution of the bands could provide information about the formation of those species. ${ }^{43}$ Additionally, a detailed time-resolved photophysical characterization of the LG@MOF composites will be essential to determine the presence of those aggregates. According to the Kasha's excitonic theory, the $\mathrm{S}_{1} \rightarrow \mathrm{S}_{0}$ is a forbidden transition for the H-aggregates, while in the case of the J-aggregates it will be less energetic than for the monomers. ${ }^{216,217}$ Considering the emission lifetimes and contributions, apart from the emission lifetime of the monomers, it is plausible to get two additional time components. One of them will be a very short emission lifetime $\left(\tau_{1}\right)$ that can be attributed to the $\mathrm{H}$ aggregates, as its relaxation is a forbidden transition. The second additional component $\left(\tau_{2}\right)$ 
whose amplitude (and/or contribution) will rise to the reddest part of the emission spectrum will correspond to the lifetime of the J-aggregates, as these will emit in the lower energy region.

The foregoing observations have been well demonstrated for a series of RhB@ZIF-71 composite materials. ${ }^{43}$ In this study, Zhang et al. prepared 3 sample batches with an increasing concentration of the $\mathrm{RhB}$ dye. Firstly, the emission spectra were red-shifted with the concentration, while the excitation spectra showed a broadening, reflecting the possible formation of both the H-and J-aggregates (Figure 24). Then, the authors carefully investigated the time-resolved photodynamics of those materials. In all the samples, it was reported a multiexponential decaying behavior, giving the three time components of: $\tau_{1}=0.28 \mathrm{~ns}, \tau_{2}=$ 1.2-2.1 ns, and $\tau_{3}=3.4-3.9 \mathrm{~ns}$. The shortest time component $\left(\tau_{1}\right)$ was assigned to the $\mathrm{H}-$ aggregates, the intermediate component $\left(\tau_{2}\right)$ attributed to the J-aggregates, while the longest component $\left(\tau_{3}\right)$ was the emission lifetime of the monomers. ${ }^{43}$ Clearly, the contribution of the $\tau_{1}$ and $\tau_{2}$ components (H- and J-aggregates, respectively) increased for the composites with a higher concentration of $\mathrm{RhB}$ guests, hence unequivocally proving the formation of a growing population of aggregates upon rising the amount of dyes in the ZIF-71 MOF. Very similar results were also reported after the encapsulation of an increasing amount of NR dye in the AlITQ-HB MOF. ${ }^{144}$ In addition to the expected red-shift of the emission spectra, the authors reported a multiexponential photobehavior with three lifetimes of: $\tau_{1}=0.71-0.26 \mathrm{~ns}, \tau_{2}=$ 2.59-1.23 ns, and $\tau_{3}=5.1-3.15 \mathrm{~ns}$, assigned to the emission lifetime of the H-aggregates, Jaggregates, and NR monomers, respectively. ${ }^{144}$ 
a)

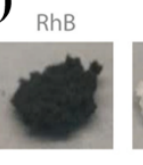

c)

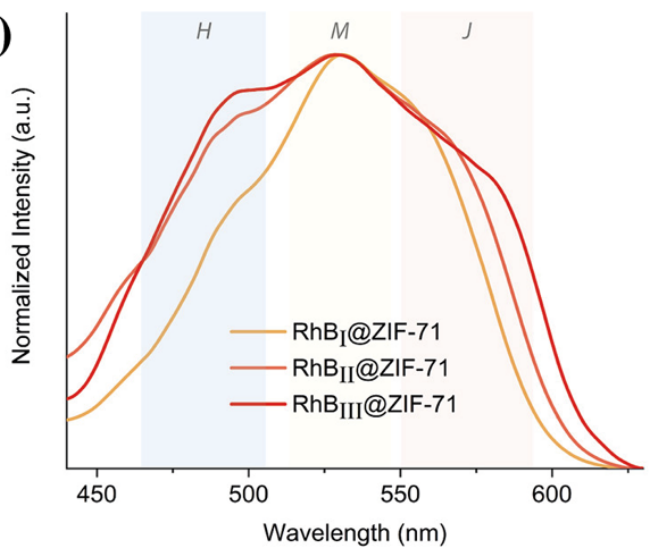

b) $)_{\text {RhB }}$

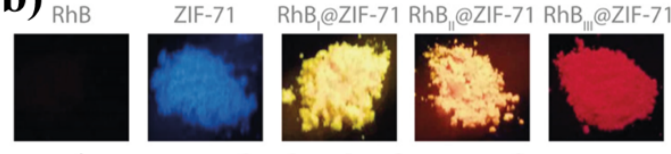

d)

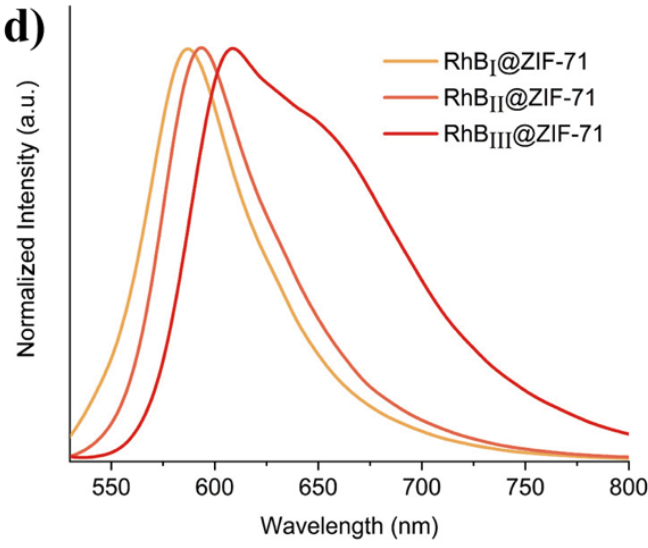

e)
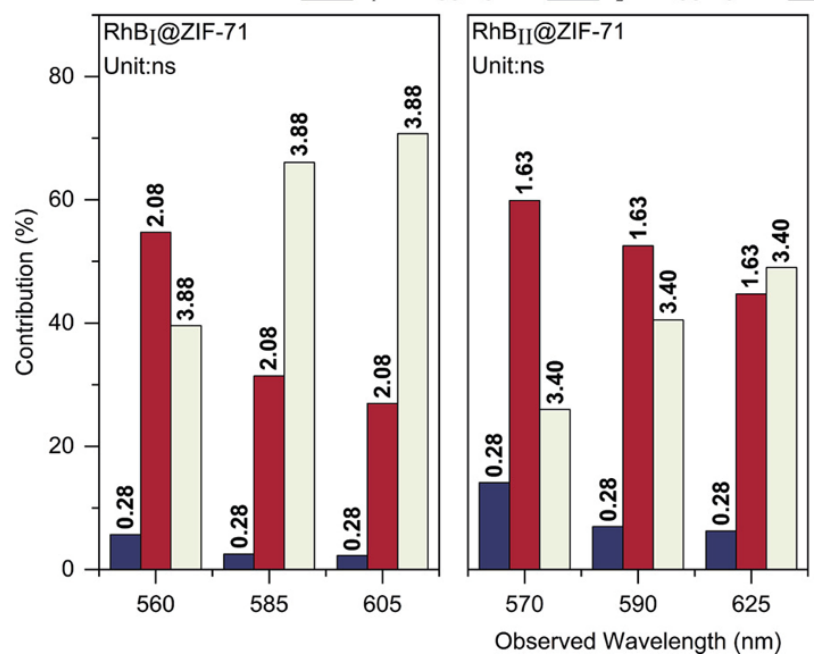

RhB III@ZIF-71 Unit:ns

Figure 24. Photographs of the RhB, ZIF-71, and RhB@ZIF-71 composites with different RhB concentrations observed under (a) daylight and (b) UV excitation $(365 \mathrm{~nm})$. (c) Normalized excitation spectra $\left(\lambda_{\mathrm{em}}=650 \mathrm{~nm}\right)$ and $(\mathrm{d})$ normalized emission spectra $\left(\lambda_{\mathrm{ex}}=515 \mathrm{~nm}\right)$ of the different RhB@ZIF-71 composites. (e) Time-resolved data of RhB@ZIF-71 obtained using three different $\mathrm{RhB}$ concentrations, showing the lifetimes and contributions of the monomer, $\mathrm{H}$ - and J-aggregate species. Adapted from ref. 42. Copyright 2020 American Chemical Society.

A similar multiexponential behavior was also reported for the fluorescein@ZIF-8 system. At lower guest concentrations, the authors reported a biexponential decay owing to the emission of the well isolated monomers and J-aggregates, while at higher guest concentrations, the decays become multiexponential with three lifetimes of $\tau_{1}=1.1 \mathrm{~ns}, \tau_{2}=3-3.7 \mathrm{~ns}$, and $\tau_{3}=$ $6.5 \mathrm{~ns}$, attributed to the emission lifetime of the H-aggregates, J-aggregates, and monomers, respectively. ${ }^{83}$ These reports are clear examples of how a detailed photodynamic characterization of LG@MOFs is essential for the control and manipulation of the composite's 
luminescent behavior, and therefore, enabling fabrication of bespoke composite systems with precisely engineered properties.

\section{POTENTIAL APPLICATIONS AND DEVICES INTEGRATING LG@MOFs}

Because of their vastly tailorable luminescent properties and stimuli-responsive behavior, the LG@MOF systems have the potential to be employed in new innovative technological applications, as summarized in Figure 25. In this section, we shall focus the discussion on emerging photonic applications in the fields of chemical, temperature, pressure sensing, energy conversion and solid-state lighting. In the vibrant field of MOF sensors, ${ }^{10,19,218,219}$ access to bespoke LG@MOF systems will offer an innovative platform for accomplishing unconventional host-guest interactions via nanoscale confinement effect. Exceptionally, one should expect to see a growing number of new sensing applications or enhanced device performance emerging from the development of LG@MOF composites.

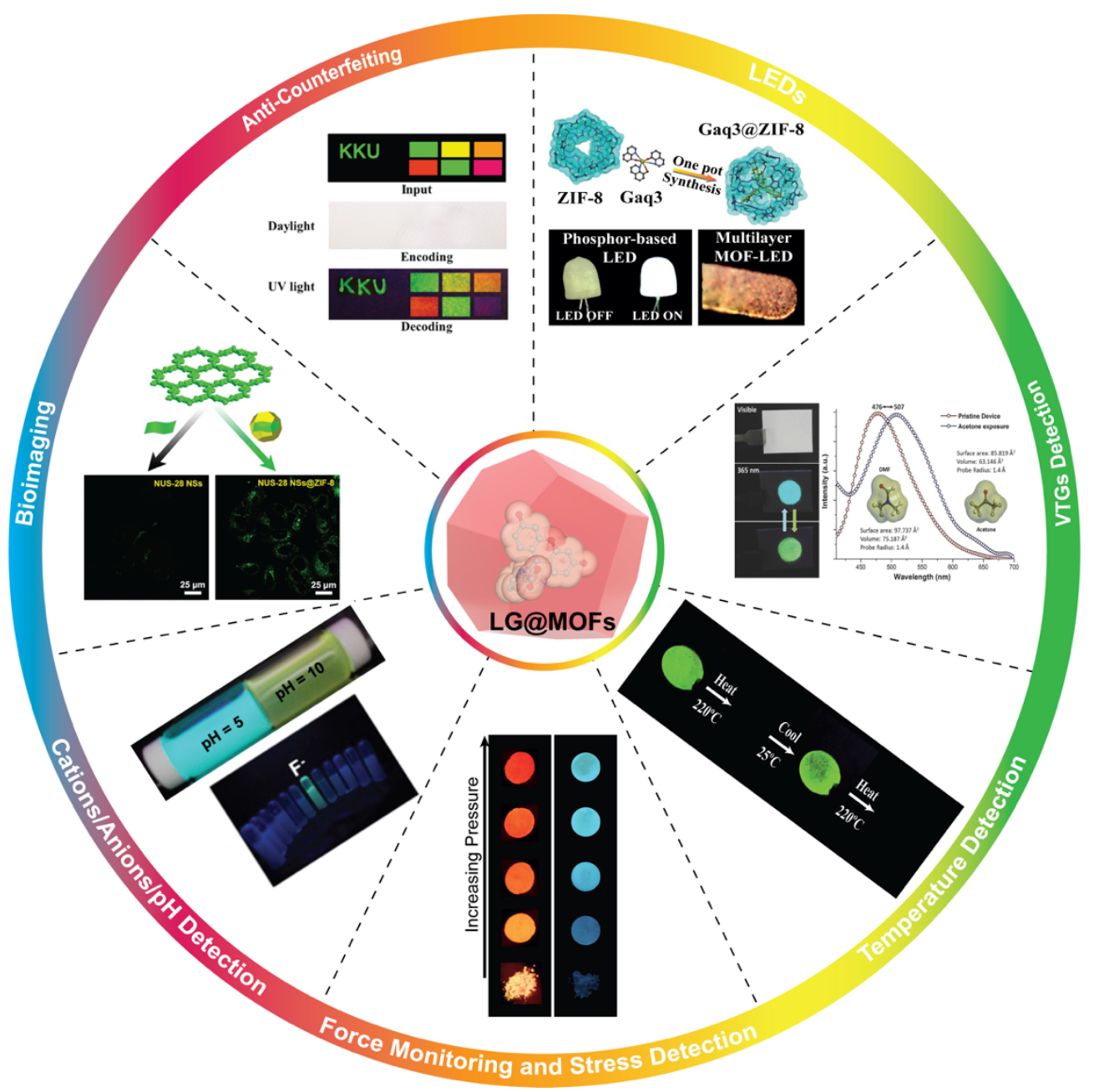


Figure 25. Emergent applications and potential uses of novel LG@MOF materials by harnessing their vast photophysical and photochemical properties to yield smart optical sensors, photo switches, and stimuli-responsive photonics. VTG = volatile organic compounds, toxic chemicals and gases. Inset figure for LEDs adapted from ref. 37. Copyright 2020 John Wiley \& Sons. Inset figure for VTG detection adapted from ref. 35. Copyright 2017 John Wiley $\&$ Sons. Inset figure for temperature detection adapted from ref. 225. Copyright 2020 Elsevier. Inset figure for force monitoring and stress detection adapted from ref. 43 (Copyright 2020 American Chemical Society) and ref. 240. Inset figure for anion sensing adapted from ref. 243. Copyright 2018 American Chemical Society. Inset figure for bioimaging adapted from ref. 147. Copyright 2019 American Chemical Society. Inset figure for anti-counterfeiting adapted from ref. 112. Copyright 2019 American Chemical Society.

Currently, the reported sensors incorporating LG@MOF materials can be roughly divided into two categories: the "turn-off" type and the "turn-on" type. When encountering a target condition or external stimulus (e.g., temperature, stress, $\mathrm{pH}$, charge), the turn-off type sensor responds by decreasing its luminescent intensity, whereas the turn-on type sensor responds to the stimuli by increasing its luminescence. It is projected that fluorescent turn-on sensing holds the key to the future sensors development, ${ }^{220,221}$ because various environmental factors, such as water/moisture, may also diminish the luminescent intensity of LG@MOF, thereby interfering with the signal output from turn-off type sensors. Further to the luminescent intensity changes, some of the turn-off and turn-on sensors constructed from LG@MOF could demonstrate a peak wavelength shift or exhibit a dual emission. The change of peak wavelength gives an intuitive visual output that is easily perceptible to the naked eye, while the dualemission behavior can be harnessed for engineering a self-calibrating device for mitigating any unwanted effects caused by the external interferences.

\subsection{Volatile Organic Compounds, Toxic Chemicals and Gases (VTGs) Detection}

Industrial activities and the daily life of modern society will inevitably use and emit a lot of volatile organic compounds (VOCs), toxic chemicals and gases (VTGs). With the rapid urbanization around the world, the negative impact of VTGs on the environment is becoming more pronounced, ${ }^{8}$ and concomitantly, the demand for high-performance VTG sensors also increases for hazard detection and risk mitigation.

Among the many potential VTG-sensing materials being developed, the LG@MOF system appears as one of the most promising in the field. For example, the recently developed RhB@ZIF-71 crystals exhibit fluorochromic VOC detection ability. ${ }^{43}$ It was reported that when RhB@ZIF-71 was exposed to certain VOCs, especially $N, N$-dimethylformamide (DMF), its luminescence becomes significantly quenched because the encapsulated RhB guest 
transformed to non-fluorescent lactone in a polar aprotic solvent. While a typical turn-off type sensing behavior has been shown here, this material is still far from making an actual VOC sensor since it will need better selectivity and inertness from environmental factors to afford practical use. Therefore, research based on the dual-emission LG@MOF system is particularly relevant, whose self-calibration property can be exploited to eliminate external interference through a ratiometric sensing approach. For example, Chen et al. ${ }^{222}$ have developed a Rh6G@MOF system (rhodamine 6G@ $\left[\left(\mathrm{NH}_{2} \mathrm{Me}_{2}\right)\left[\mathrm{Zn}_{3}\left(\mu_{3}-\mathrm{OH}\right)(\mathrm{tpt})(\mathrm{TZB})_{3}\right](\mathrm{DMF})_{12}\right]_{\mathrm{n}}$, tpt = 2,4,6-tri(4-pyridyl)-1,3,5-triazine, $\mathrm{H}_{2} \mathrm{TZB}=4$-(1H-tetrazol-5-yl)benzoic acid). This material exhibits two emission peaks originating from the tpt ligand $(363 \mathrm{~nm})$ and Rh6G $(580 \mathrm{~nm})$, respectively. When exposed to 2,4,6-trinitrophenol (TNP, a toxic pollutant and highly explosive molecule), the luminescence from the ligand becomes significantly quenched, while the response of Rh6G was only minimally affected. By tracking the intensity ratios derived from the two emission peaks, the TNP molecules can be detected effectively (Figure 26a). Furthermore, the authors found that the Rh6G@MOF could selectively detect TNP among many non- and most nitroaromatic compounds (Figure 26b). Prior to this study, all the MOFbased TNP sensors reported were simply the type-off type, this work therefore marks a significant development in the field by demonstrating the promise for building a ratiometric fluorescent sensor by leveraging the LG@MOF concept. In the same way, Yin et al. ${ }^{223}$ reported a $\mathrm{Ru} @ \mathrm{MIL}-\mathrm{NH}_{2}$ system capable of ratiometric fluorescence sensing of water in organic solvents. This work demonstrates the dual emission characteristics having a relatively fast response time of $\sim 1$ minute, further highlighting the potential of the LG@MOF concept. 

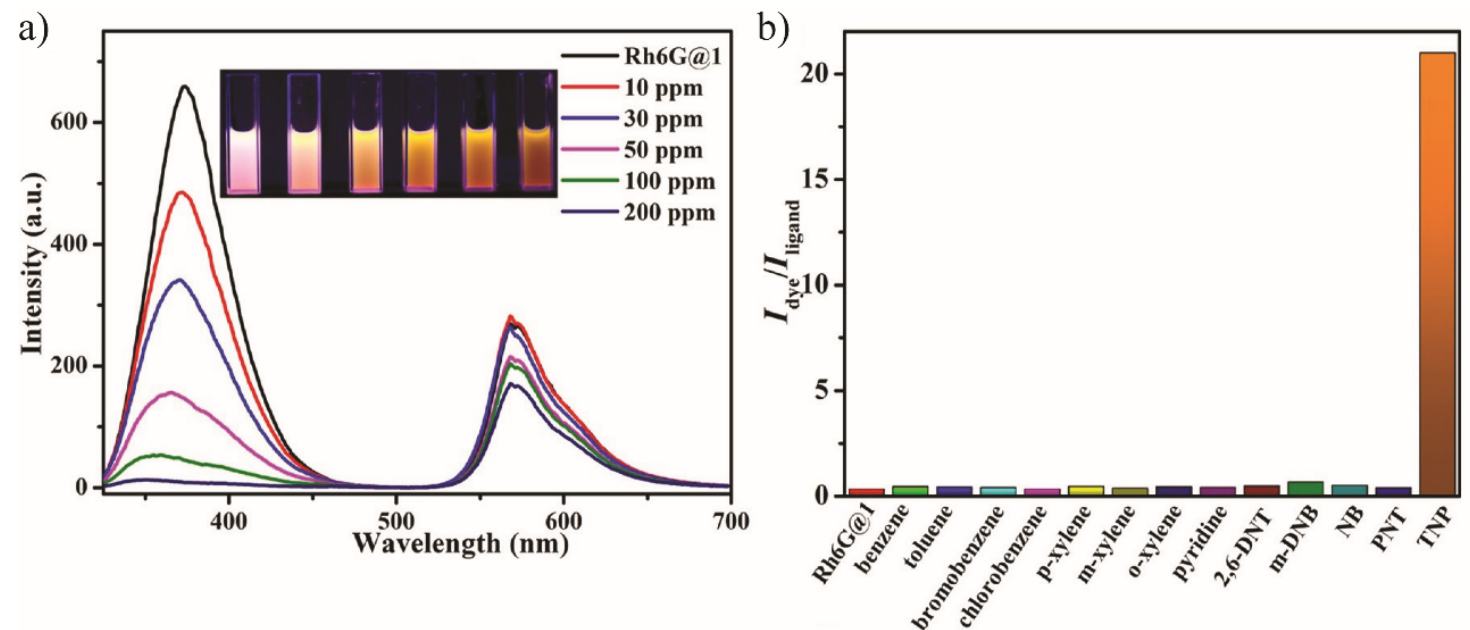

c)

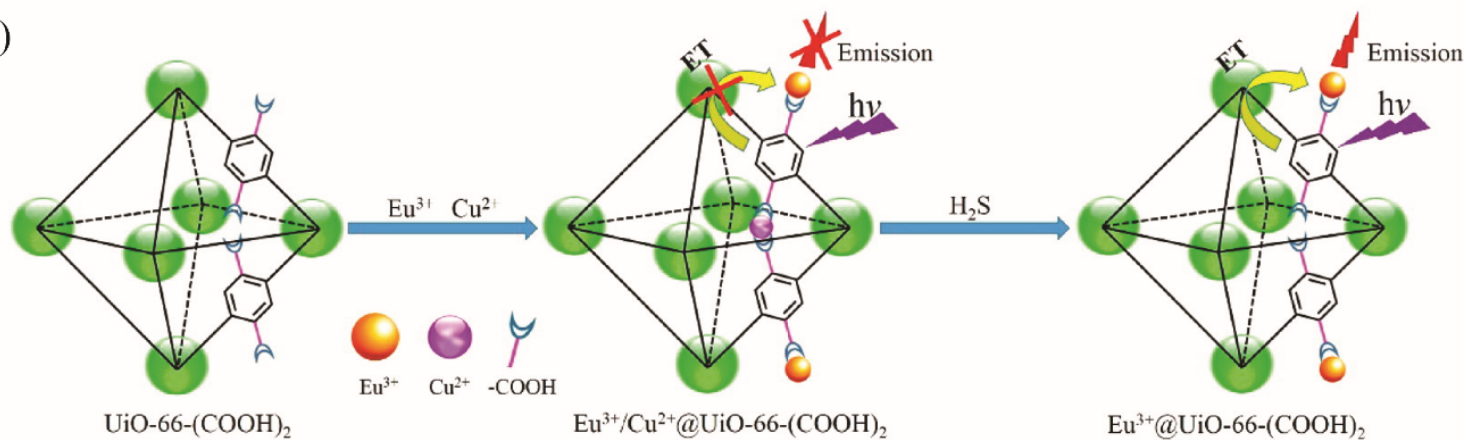

Figure 26. (a) Emission spectra for Rh6G@MOF at different concentrations of TNP [MOF = $\left.\left[\left(\mathrm{NH}_{2} \mathrm{Me}_{2}\right)\left[\mathrm{Zn}_{3}\left(\mu_{3}-\mathrm{OH}\right)(\mathrm{tpt})(\mathrm{TZB})_{3}\right](\mathrm{DMF})_{12}\right]_{\mathrm{n}}\right]$. (b) The peak-height ratio of dye to ligand after addition of $200 \mathrm{ppm}$ of various analytes. Adapted with permission from ref. 223. Copyright 2017 American Chemical Society. (c) Schematic illustration of the fluorescence detection mechanism of $\mathrm{Eu}^{3+} / \mathrm{Cu}^{2+} @ \mathrm{UiO}-66-(\mathrm{COOH})_{2}$. Adapted with permission from ref. 224. Copyright 2016 American Chemical Society.

The turn-on type response of LG@MOF sensors used to detect VTGs is relatively rare, thus far this requires Ln-based materials. For example, Zhang et al. reported a Eu ${ }^{3+} / \mathrm{Cu}^{2+} @ \mathrm{UiO}-$ 66- $(\mathrm{COOH})_{2}$ system showing turn-on and ratiometric sensing for $\mathrm{H}_{2} \mathrm{~S} .{ }^{224}$ When not in contact with the target $\mathrm{H}_{2} \mathrm{~S}$ molecules, the system shows dual-emission characteristics of both $\mathrm{Eu}^{3+}$ and the ligand, and there was an energy transfer from the ligand $\left(\mathrm{H}_{4} \mathrm{btec}=1,2,4,5\right.$ benzenetetracarboxylic acid) to the $\mathrm{Eu}^{3+}$ ions, this phenomenon is called the antenna effect, see the mechanism depicted in Figure 26c. The $\mathrm{Cu}^{2+}$ in the system could interact with the Lewis basic carboxylic oxygen sites to form $\mathrm{O}-\mathrm{Cu}-\mathrm{O}$ through the cooperative effect, and this interaction will restrain the antenna effect, causing a weak $\mathrm{Eu}^{3+}$ and a strong ligand emission. When the system is exposed to sulfide, $\mathrm{Cu}^{2+}$ (a soft acid), would react with the sulfide (a soft base), destroying the $\mathrm{O}-\mathrm{Cu}-\mathrm{O}$ structure. Therefore, the greater antenna effect leads to rise in the $\mathrm{Eu}^{3+}$ luminescence but a decline of emission intensity associated with the ligand. This type of Ln@MOF system can retain the intrinsic properties of encapsulated $\mathrm{Ln}^{3+}$, and may help to 
overcome the design and synthesis difficulties due to the higher coordination numbers and the more variable nature of the $\mathrm{Ln}^{3+}$ coordination sphere commonly encountered in the more conventional Ln-based MOFs. ${ }^{53}$

\subsection{Temperature Sensing and Non-Invasive Thermometry}

The temperature sensor is one of the most commonly used sensors in our daily life, and it can be roughly divided into the contact type and non-contact type. However, these two types cannot meet all the practical engineering requirements. Contact type temperature sensors must be put in contact with the target to acquire a reading. While non-contact types, such as infrared (IR) thermometers, can be easily affected by the environment, and they are neither suitable for measuring fine-scale microscopic objects nor for measuring the temperature of the interior of objects.

In order to address these shortcomings, some researchers have turned their attention to luminescent thermometers. Simply put, one can place the fluorescent thermochromic material in the vicinity of the target, which will non-invasively measure the temperature of the surrounding by detecting the change in its luminescent behavior. ${ }^{225}$ Because of the various forms and nano size of luminescent materials, this enables one to perform challenging temperature measurements in unconventional settings. ${ }^{12}$

The LG@MOF is a good system for making luminescent temperature sensors, but most of LG@MOF based sensors are the turn-off type. That is because the increase in temperature will cause the non-radiative decay of the emitter to increase, leading to a decrease in the overall luminescent intensity. For example, $\mathrm{Hu}$ et al. ${ }^{28}$ used a MOF host, $\left[\left(\mathrm{CH}_{3}\right)_{2} \mathrm{NH}_{2}\right]^{+}\left[\mathrm{Zn}_{4}\left(\mu_{4}-\right.\right.$ $\mathrm{O})(\mathrm{NTB})_{2}\left(\mathrm{NO}_{2} \text {-bdc }\right)_{0.5}$ ] 3DMA ( $\mathrm{NTB}=4,4$ ', 4' '-nitrilotrisbenzoic acid, $\mathrm{NO}_{2}$-bdc = 2-nitro-4benzenedicarboxylicacid), to encapsulate RhB guest and obtain a RhB@MOF system. This system exhibits the characteristics of traditional RhB-based temperature sensing, where its luminescent intensity decreases as a function of temperature rise.

Further to retaining the temperature sensitivity of the luminescent guest itself, more importantly, the LG@MOF may impart the ability of peak wavelength shift on the fluorophore because of the pore confinement effect (Section 5.4). For example, the RhB@ZIF-71 ${ }^{43}$ also exhibits a turn-off thermochromic response. Compared with pure RhB, RhB@ZIF-71 exhibits a red-shift of its emission spectrum with an intensity that decreases linearly as a function of temperature as shown in Figure 27a-b. That is because the MOF pore imposes different caging effects on the $\mathrm{RhB}$ monomers, J-aggregates, and $\mathrm{H}$-aggregates. The J-aggregates are relatively 
larger and receive a stronger protection due to pore confinement. Therefore, when temperature increases, the relative luminescent intensity of the J-aggregates decreases less rapidly, and this effect produces a red-shift of the overall emission. In contrast, all other RhB-based temperature sensors reported to date could only show the common decline in their emission intensity, ${ }^{226,227}$ without demonstrating a peak shift observed in the RhB@ZIF-71 system.. ${ }^{43}$ The above finding shows that the guest-host interaction of LG@MOF system can be harnessed to broaden the potential applications of conventional fluorophores to accomplish unconventional temperature sensing capabilities.
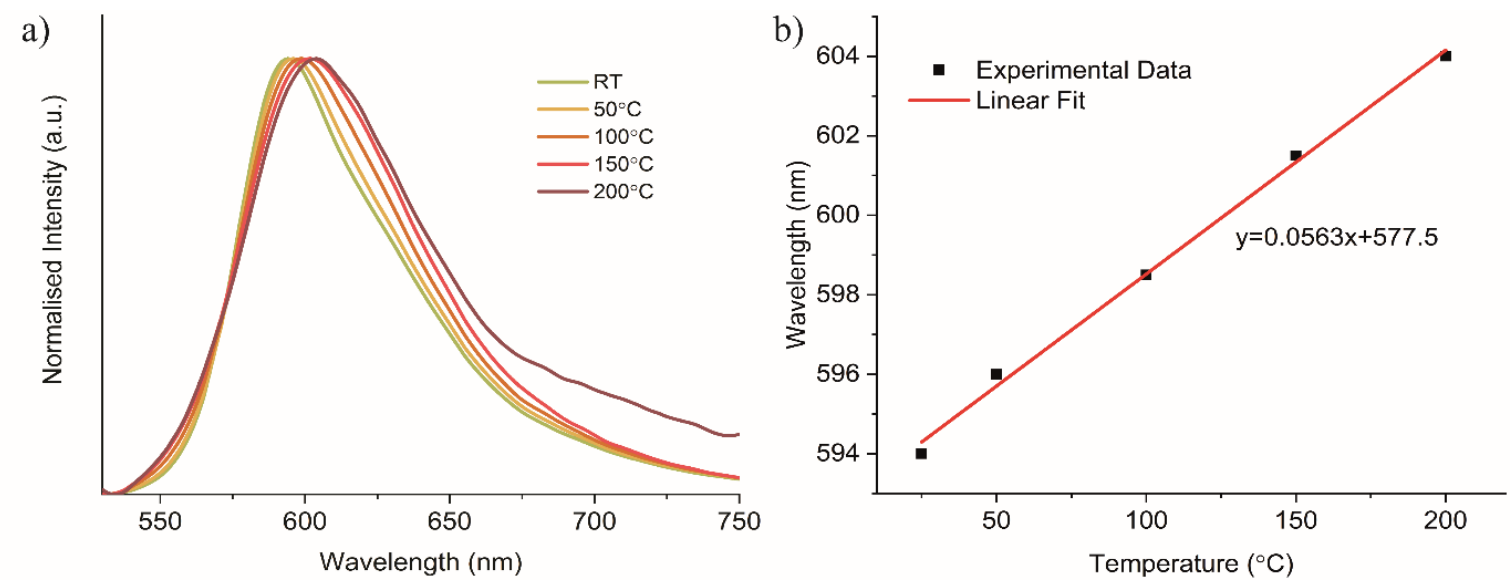

c)

d)
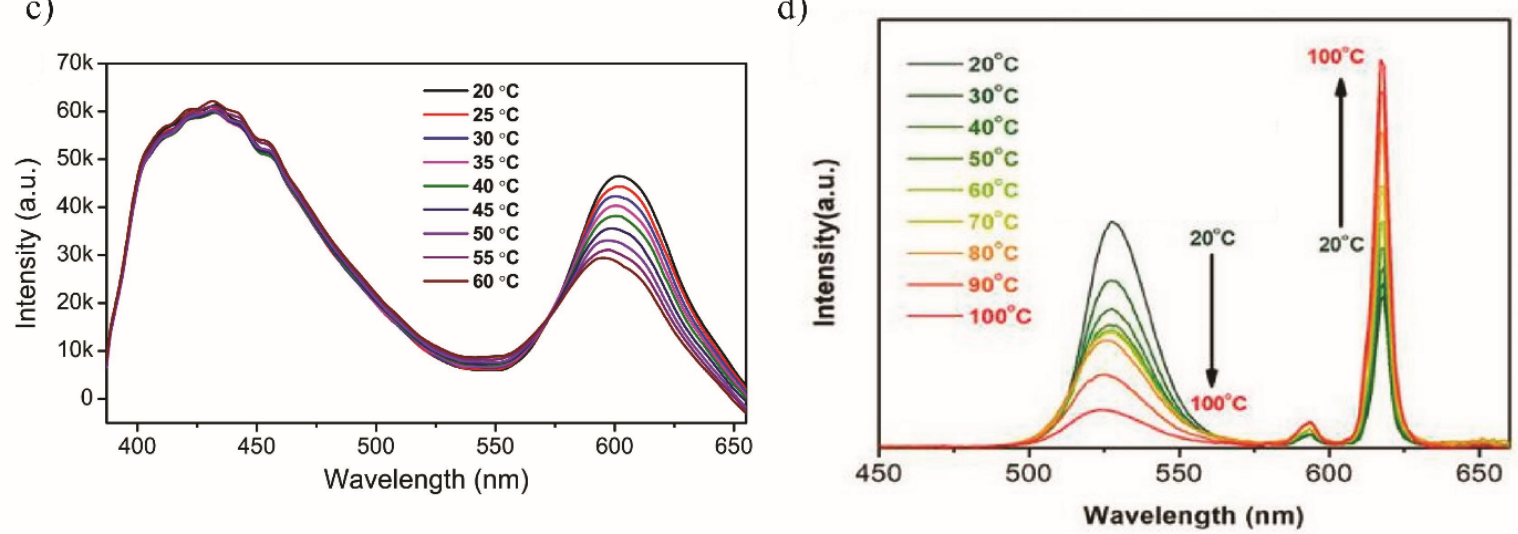

Figure 27. (a) Normalized emission spectra of RhB@ZIF-71 when temperature rises from room temperature to $200^{\circ} \mathrm{C}$. (b) Linear relationship of the emission peak wavelength of RhB@ZIF-71 as a function of temperature. Adapted from ref. 43. Copyright 2020 American Chemical Society. (c) Temperature-dependent photoluminescence emission spectra of Rh101@UiO-67. Adapted with permission from ref. 230. Copyright 2018 Elsevier. (d) Temperature-dependent photoluminescent spectra of CsPbBr3@Eu-BTC in the temperature range of 20-100 ${ }^{\circ} \mathrm{C}$. Adapted with permission from ref. 121. Copyright 2020 American Chemical Society.

The LG@MOF system can bestow unique sensing ability onto some luminescent guests or fluorophores which, in the pristine form, do not exhibit any temperature sensing properties. 
For example, the Rhodamine 101 (Rh101) dye is a thermally insensitive material, due to its structural rigidity. ${ }^{228,229}$ Zhou et al. ${ }^{230}$ reported a Rh101@UiO-67 system, which has both Rh101 and UiO-67 luminescence, but the emission from the former reduces with increasing temperature as shown in Figure 27c. The authors proposed that this phenomenon was related to the back energy transfer from the dye molecules to the linkers and the increase in temperature would increase this effect, causing the intensity to decline in Rh101. Remarkably, because the emission from the UiO-67 host stays unchanged with temperature, the system becomes as a self-calibrating sensor.

There are some lanthanide-based LG@MOFs that can perform turn-on temperature sensing. Unlike pure Ln-MOFs, it is possible to introduce a turn-off type LG into them, which will yield a self-calibrating system and improve the temperature resolution. For example, Liu et al. ${ }^{121}$ encapsulated a perovskite $\mathrm{CsPbBr}_{3}$ into an Ln-MOF (Eu-BTC) host and the obtained $\mathrm{CsPbBr}_{3} \mathrm{C} @ \mathrm{Eu}-\mathrm{BTC}$ exhibits both the characteristic emission peaks of $\mathrm{Eu}^{3+}$ and $\mathrm{CsPbr}_{3}$. With the increase in temperature, as shown in Figure 27d the emission of $\mathrm{Eu}^{3+}$ rises and that of $\mathrm{CsPBr}_{3}$ falls, resulting in a turn-on type response jointly with self-calibrating feature. The authors claimed that although $\mathrm{Eu}^{3+}$ in other composites had a very low-temperature response due to the weak coupling effect, the high luminescent efficiency of $\mathrm{CsPbBr}_{3}$ and the confinement effect of Eu-BTC host significantly improved the temperature sensing performance of $\mathrm{Eu}^{3+} .121$ This improvement once again illustrates the potential benefits for deploying bespoke LG@MOF composite systems.

\subsection{Mechanochromic Sensors for Force Monitoring and Stress Detection}

Fluorescent mechanochromic sensors have attracted much attention because of their potential implementation in mechanical stress/strain-based adaptive devices and for the visual detection of material failure. At the core of the mechanofluorochromic sensor is a fluorescent sensing material that changes its luminescent response as a function of the imposed force (stress), deformation (strain), or pressure. Particularly, there are plentiful reports available on the mechanofluorochromic behavior of polymeric systems, organic compounds, and metal complexes. ${ }^{231,232}$ Conversely, studies on mechanochromic MOFs are relatively limited, ${ }^{233-237}$ and fewer still if based on the concept of mechanochromic Guest@MOF systems. ${ }^{38,43}$

The typical mechanisms that control the emission of a mechanochromic material is the mechanically-induced deformation of the structure to yield excimer formation, ${ }^{238}$ phase transformation under stress, or via aggregation induced emission (AIE) under pressure. ${ }^{239}$ It 
has been proposed that high-efficiency mechanochromic materials are more likely to be obtained when the mechanism is driven by structural changes from physical means rather than by chemical means. ${ }^{231}$ Thus, the physical caging of fluorophores by long-range nanoconfinement in LG@MOF system makes it an ideal candidate for achieving mechanochromism. For example, the perylene@ZIF-8 system reported by Chaudhari et al. ${ }^{38}$ shows a reversible mechanochromic response with an emission wavelength shift. When this material was not stressed, under UV, it emits in blue (442 nm) but after compression it switches to a green emission (502 $\mathrm{nm}$ ). Subsequently, the initial blue emission can be regained when the compressed pellet was ground into a powder form to release residual stress.

Another example concerns the RhB@ZIF-71 system that exhibits a turn-off mechanochromic effect, see Figure 28a. ${ }^{43}$ This mechanism involves not only the enhanced $\pi^{-} \pi$ interaction, but it has been hypothesized that an increasing pressure generates a tighter formation of J-aggregates coupled with the switching of J-to-H aggregates within the pores, resulting in a red-shifted emission.

a)

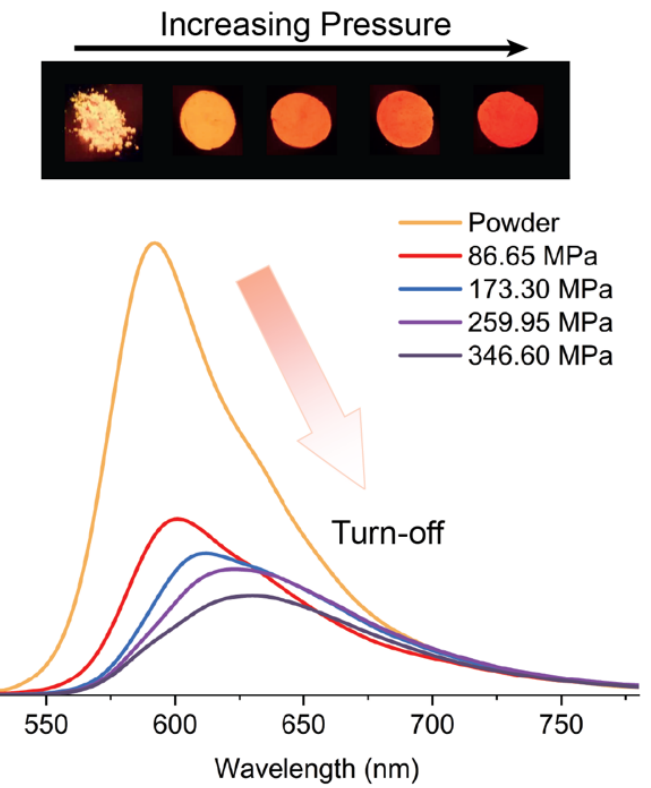

b)

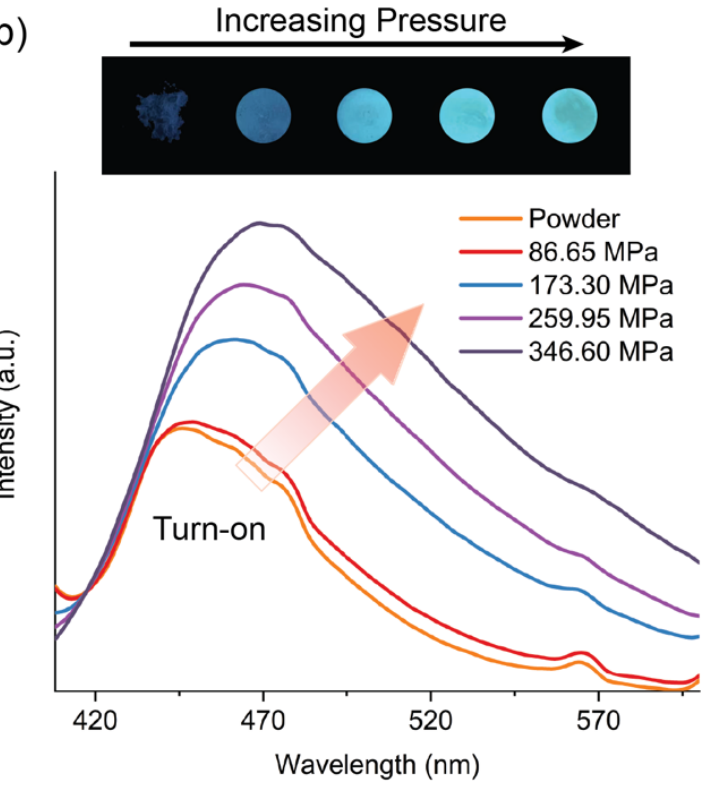

Figure 28. Emission spectra showing the turn-off and turn-on type mechanofluorochromic response of (a) RhB@ZIF-71. Adapted from ref. 43. Copyright 2020 American Chemical Society. and (b) TPE@ZIF-71, respectively, when samples were subjected the effect of pelleting pressure. Adapted from ref. 240. The diameter of the pellet is $\sim 10 \mathrm{~mm}$. Adapted from ref. 240. Copyright 2022 Elsevier.

Leveraging the nanoconfinement effect conferred by MOF structures, recently it has been shown that the turn-on type mechanochromism of LG@MOF can also be accomplished. 
The study concerns the TPE@ZIF-71 system (TPE = tetraphenylethylene; an AIE molecule), where the vibrations of the TPE guest molecules were found to be restricted when confined within ZIF-71 pores and under pressure, this mechanism generates a turn-on mechanochromic response (Figure 28b) ${ }^{240}$ It must be emphasized that pristine (unconfined) RhB, perylene, and TPE molecules are not mechanochromic by themselves under the same test conditions. The foregoing exemplars support the notion that the ordered framework structure of the MOF host could help to direct the mechanical response of the LG@MOF system. Significantly, the physical structural changes undergone by LG@MOFs subject to mechanical deformation or pressure can be translated into an efficient mechanofluorochromic response, potentially yielding both kinds of turn-on/-off mechanosensors by design.

\subsection{Cations and Anions Sensing, Optical pH Detection}

The research on cation/anion sensing is one of the most popular directions in the luminescent MOF field, but most studies are related to the turn-off type. Generally, one can directly harness the properties of the guest itself in the LG@MOF system to attain sensing behavior. For example, Asadi et al. ${ }^{34}$ developed a core-shell LG@MOF system, comprising polyethylene glycol (PEG)-capped ZnS QDs@ZIF-67. This system showed sensitivity to $\mathrm{Cu}^{2+}$ detection. The mechanism involves the oxygen atoms on the hydroxyl groups of PEG-ZnS itself, which form a complex with $\mathrm{Cu}^{2+}$ thus quenching the luminescence of PEG-ZnS. Although this mechanism is not altered by MOF confinement, compared with the pure PEG$\mathrm{ZnS}$ (unencapsulated), it was found that the accumulation effect in the ZIF-67 pores further boost the sensitivity and selectivity towards the $\mathrm{Cu}^{2+}$ analyte (Figure 29a-b). Notably, research to date has shown that even if the MOF host is not directly involved in the sensing process, it still can play an important role by enhancing the ion sensing ability of the encapsulated guest, thereby raising the performance of the LG@MOF system as a whole. 
a)

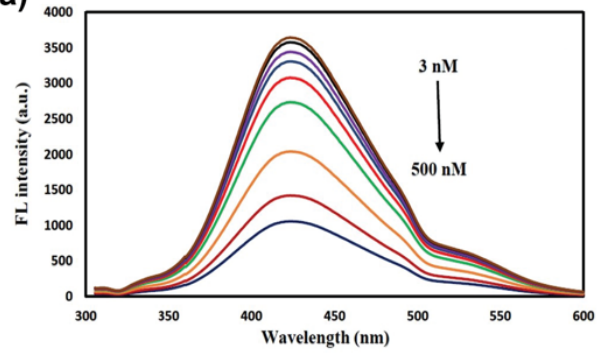

c)

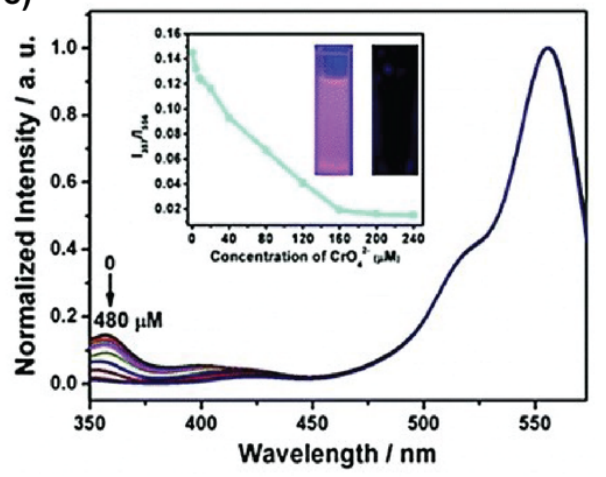

e)

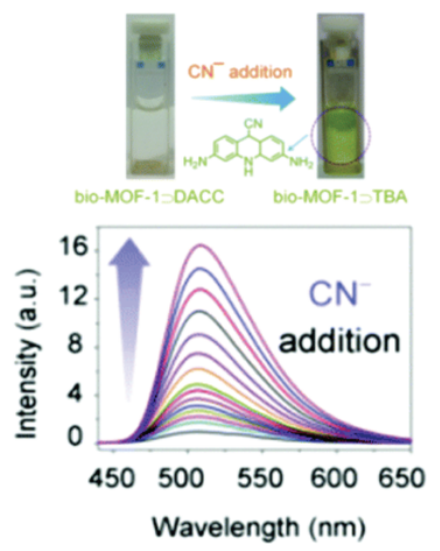

b)

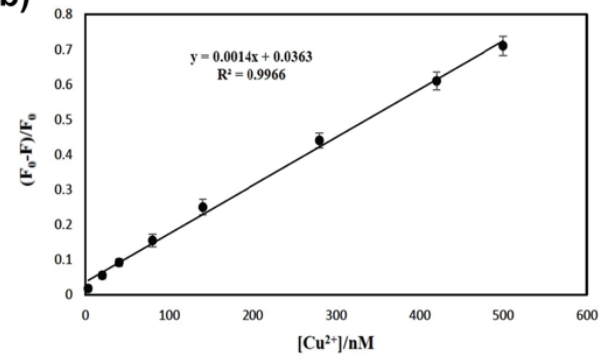

d)

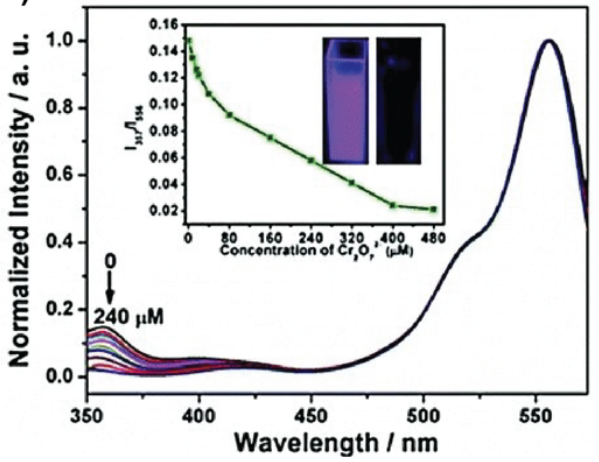

f)

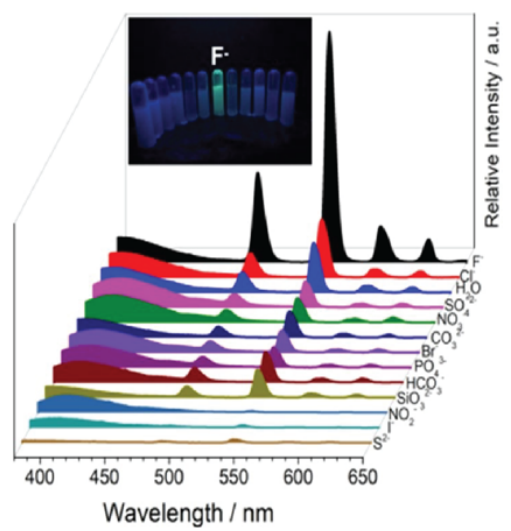

Figure 29. (a) Fluorescence spectra of PEG-ZnS QD@ZIF-67 in the presence of a series of different concentrations of $\mathrm{Cu}^{2+}$ ions from 3 to $500 \mathrm{nM}$. (b) The linear relationship between the fluorescent intensity and $\mathrm{Cu}^{2+}$ concentrations from 3 to $500 \mathrm{nM}$. Adapted with permission from ref. 34. Copyright 2019 Elsevier. (c-d) Fluorescence excitation spectral changes of RhB@ZIF90 with the addition of (c) $\mathrm{CrO}_{4}{ }^{2-}$ and (d) $\mathrm{Cr}_{2} \mathrm{O}_{7}{ }^{2-}$. Adapted with permission from ref. 241. Copyright 2018 Royal Society of Chemistry. (e) Visual change in the supernatant solution upon addition of cyanide ions to DAAC@bio-MOF-1 and changes in fluorescence intensity via a turn-on response upon addition of $\mathrm{CN}^{-}$ions to DAAC@bio-MOF-1. Adapted with permission from ref. 242. Copyright 2017 Royal Society of Chemistry. (f) Emission spectra of $\mathrm{Tb}^{3+} @ \mathrm{Zr}$ MOF dispersed in different anion solutions. Adapted with permission from ref. 243. Copyright 2018 American Chemical Society.

Many studies have indeed made full use of the dual-emission properties of LG@MOF to fabricate sensors with self-calibrating capability for the detection of cations and anions. For example, Jin et al. ${ }^{241}$ encapsulated RhB into the nanosized crystals of ZIF-90 and the obtained RhB@ZIF-90 system exhibited ratiometric fluorescent sensing to $\mathrm{Cr}(\mathrm{VI})$ anions (Figure 29c- 
d). This is because the UV-Vis absorption of $\mathrm{Cr}(\mathrm{VI})$ anions such as $\mathrm{CrO}_{4}{ }^{2-}$ and $\mathrm{Cr}_{2} \mathrm{O}_{7}{ }^{2-}$ covered most of the excitation spectrum of ZIF-90, and that inhibited the excitation of ZIF-90, while the excitation of $\mathrm{RhB}$ was not influenced by the $\mathrm{Cr}(\mathrm{VI})$ anions. In this way, when exposed to $\mathrm{Cr}(\mathrm{VI})$ anions, the excitation peak of ZIF-90 decreased, but that of RhB remained unchanged, thus yielding the self-calibration property.

Compared with the VTGs, temperature, and mechanochromic sensors discussed above, there are relatively more LG@MOF turn-on sensors for cations or anions. We can roughly divide this part of turn-on type sensors into 2 categories according to whether $\mathrm{Ln}^{3+}$ is used in the system or not. In principle, LG@MOF systems comprising ordinary (non- $\mathrm{Ln}^{3+}$ ) luminescent guests usually use MOF to suppress the luminescence of the guest, such that only when the target ion comes into contact, the guest will reactivate its own emission to achieve turn-on sensing. For example, the DAAC (3,6-diaminoacridinium cation)@bio-MOF-1 reported by Karmakar et al. has demonstrated the turn-on sensing of cyanide ions $\left(\mathrm{CN}^{-}\right)$, see Figure 29e. ${ }^{242}$ In terms of its mechanism, the emission of DAAC was initially being inhibited by bio-MOF-1 in the guest-host system, and when it came into contact with $\mathrm{CN}^{-}$, the $\mathrm{CN}^{-}$ would attack the $\mathrm{C} 9$ carbon of DAAC molecules to form a covalent bond. The formed DAAC$\mathrm{CN}$ was neutral and had good luminescent properties, so it would escape from the host framework and induce turn-on sensing. The principle of deploying a MOF host to suppress the luminescence of the confined guest is innovative, further research in this direction is warranted.

The second category of turn-on type cation/anion sensors being studied is based on the $\mathrm{Ln}^{3+}$ ions. In that case, the energy transfer/antenna effect is usually key for controlling the sensing performance of Ln@MOF. For instance, Zheng et al..$^{243}$ introduced $\mathrm{Tb}^{3+}$ into a $\mathrm{Zr}$ MOF to obtain the $\mathrm{Tb}^{3+} @ \mathrm{Zr}-\mathrm{MOF}$ system, whose luminescent properties are greatly enhanced when exposed to fluoride ions in an aqueous solution (Figure 29f). This is because an obvious antenna effect is established between the ligand of $\mathrm{Zr}-\mathrm{MOF}$ and $\mathrm{Tb}^{3+}$, but the antenna effect becomes negatively affected when the system is in water. When $\mathrm{F}^{-}$is present, it generates a Lewis acid-base interaction with the uncoordinated $\mathrm{Zr}^{4+}$ in the $\mathrm{Zr}-\mathrm{MOF}$ structure, restoring the energy transfer from the ligand to $\mathrm{Tb}^{3+}$ and reactivating the turn-on sensing response. Similarly, $\mathrm{Eu}^{3+} @$ UiO-66(Zr)- $(\mathrm{COOH})_{2}$ developed by Han and $\mathrm{Yan}^{244}$ has the turn-on type sensing behavior to $\mathrm{Cd}^{2+}$ for the same mechanism elucidated above.

LG@MOF materials can be developed for use as photoluminescent pH sensors. Figure 30 shows an exemplar where the dispersed ZnQ@OX-1 nanosheets ${ }^{35}$ are highly sensitive to the changing $\mathrm{pH}$ of the analyte solutions, within the tested range of $\mathrm{pH}=1$ to 10 . When subject to an acidic environment, the emission of ZnQ@OX-1 nanosheets exhibits a blue shift in the 
presence of $\mathrm{H}^{+}$donating species. Conversely, when exposed to a basic environment, the emission of ZnQ@OX-1 becomes gradually red shifted in response to the presence of the $\mathrm{H}^{+}$ extracting species. The concept of optical $\mathrm{pH}$ response evidenced here can be ascribed to the donor/acceptor host-guest interactions conferred by nanoscale confinement. In fact, the pristine ZnQ metal complex, while it is luminescent, is not $\mathrm{pH}$ sensitive when not confined in the OX-1 MOF host. Nevertheless, caution is needed as the exposure to stronger acids (e.g., $\mathrm{pH}<5$ for ZnQ@OX-1) and bases may cause decomposition of the MOF structure leading to the loss of its $\mathrm{pH}$ sensing ability. In principle, adopting MOF hosts with improved acid-basic stability may be able to extend the operational range of such optical $\mathrm{pH}$ sensors for deployment in even harsher environments.
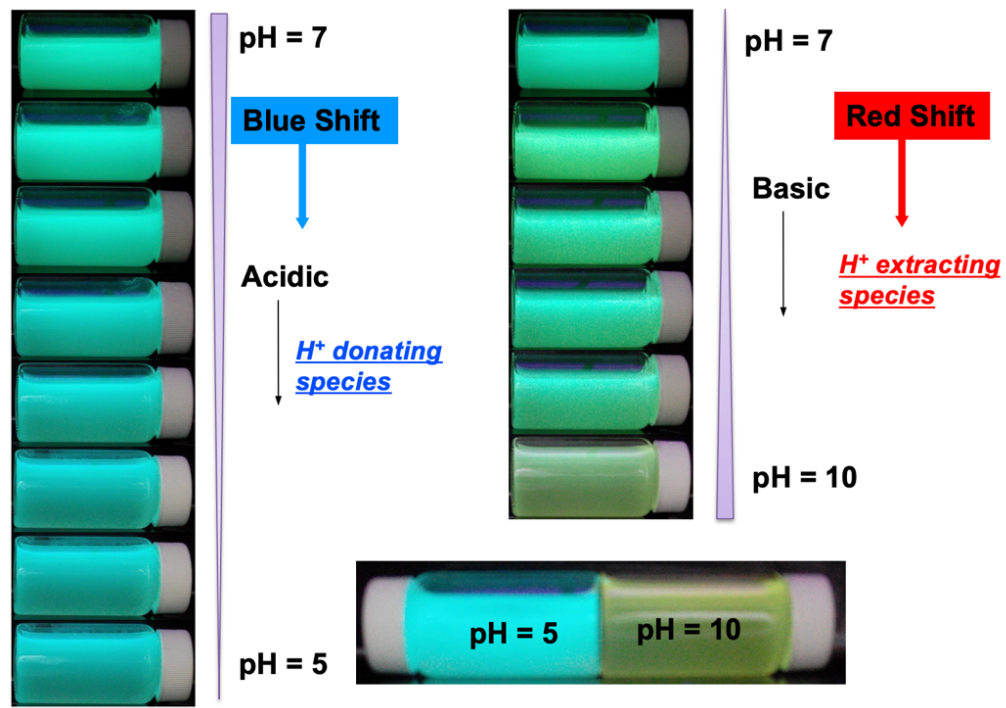

Figure 30. Optical pH sensing by utilizing the luminescent $\mathrm{ZnQ} @ \mathrm{OX}-1$ nanosheets ${ }^{35}$ suspended in a set of analyte solutions whose $\mathrm{pH}$ varies systematically from 5 to 10 .

\subsection{Bioimaging}

Bioimaging is a non-invasive method that allows the visualization of biological processes at real time, and thus, it has become an indispensable tool for monitoring biological phenomena and medical diagnosis. ${ }^{245}$ Therefore, there exists a great interest in the scientific community on developing new biomarker materials with tunable and improved abilities. Among all the proposed materials, LG@MOFs have emerged as a potential candidate for cell imaging, especially those in which the MOF has a nanometric size (nMOFs).${ }^{79,246,247}$ Additionally, the numerous possible combinations of fluorescent guests and MOFs open an exciting avenue of research in this subject. Some outstanding MOF-based fluorescent probes have been reported 
for cell-imaging, they composed of a wide variety of luminescent guests, such as organic fluorophores, ${ }^{79,248}$ porous organic nanosheets, ${ }^{145}$ metal nanoclusters, ${ }^{246,249}$ and carbon dots. ${ }^{247}$ For example, the encapsulation of Resorufin and Rhodamine 6G in the nMOF-801 and nUiO67 (Rs@nMOF-801 and R6G@nUiO-67), leads to the fabrication of LG@MOF materials that have been used for imaging the FL83B and HepG2 cells. ${ }^{79}$ Similarly, another LG@MOF material synthesized by the encapsulation of $\mathrm{RhB}$ into a hierarchical-pore Al-MOF has been successfully employed for imaging live MGC-803 cancer cells. ${ }^{248}$ In addition, the authors demonstrated the possible biodistribution of the RhB@Al-MOF in different organs, and found a major accumulation in the liver, stomach, lung and kidney, indicating that the material can be transported to all tissues by blood flow. ${ }^{248}$ Metal nanoclusters (NCs), like Ag or Au NCs, have also been incorporated into different MOFs proving their capacity for imaging cells like MCF-7 or RAW264.7. ${ }^{246,249}$ Another interesting example demonstrates how the encapsulation of porous organic nanosheets (PONs) into the ZIF-8 MOF has enhanced the ability for labelling the HeLa and MCF-7 cells. ${ }^{145}$ Meanwhile the pristine nanosheets barely showed any luminescence, upon their encapsulation into ZIF-8 the living cancer cells were easily imaged (Figure 31). ${ }^{145}$ 


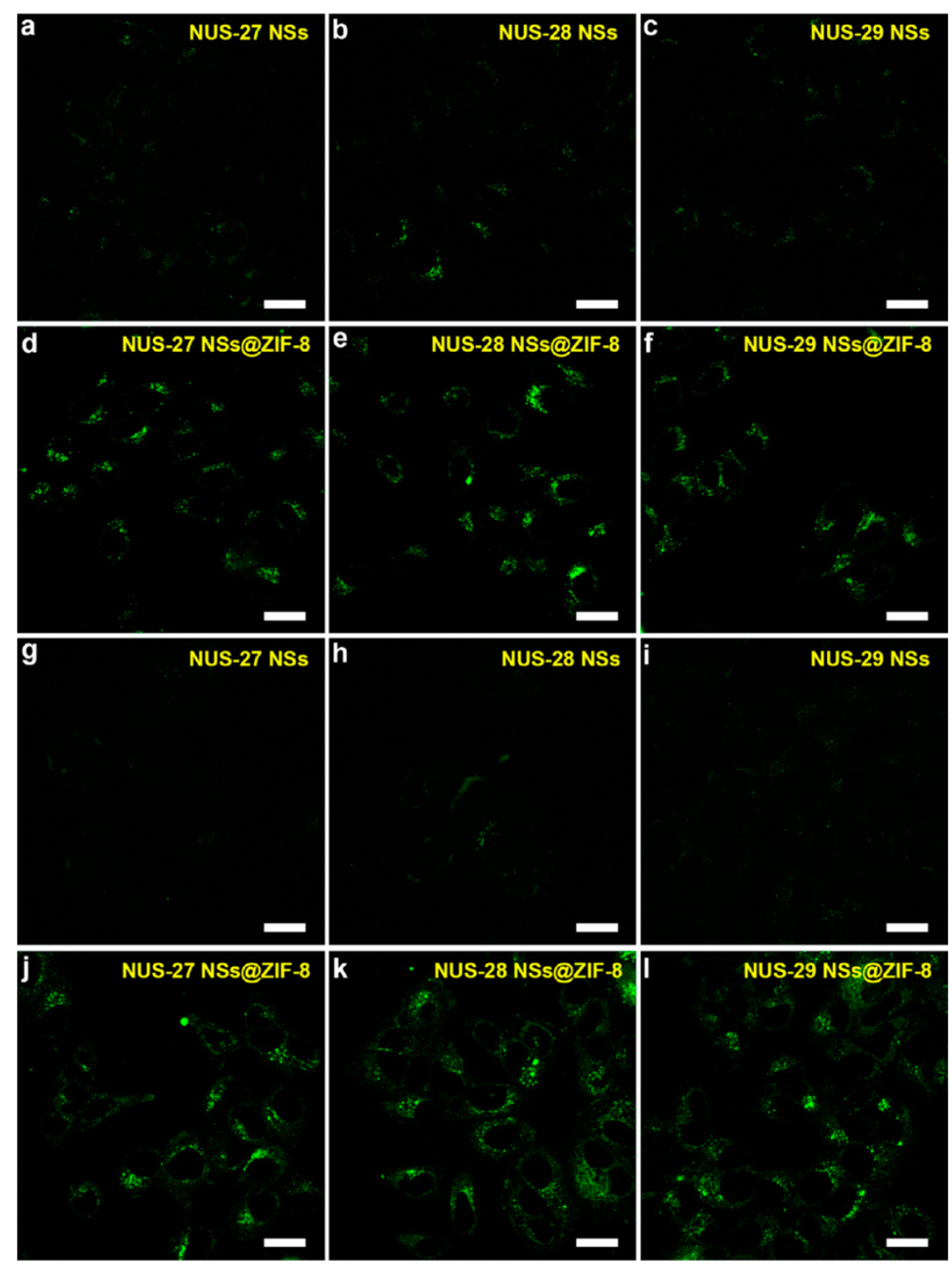

Figure 31. Confocal images of HeLa (a-f) and MCF-7 (g-l) cells using NUS-27 nanosheets (NSs) (a, g), NUS-28 NSs (b, h), NUS-29 NSs (c, i), NUS-27 NSs@ZIF-8 (d, j), NUS-28 NSs@ZIF-8 (e, k), and NUS-29 NSs@ZIF-8 (f, 1), respectively. Adapted with permission from ref. 145. Copyright 2019 American Chemical Society.

\subsection{Anti-Counterfeiting}

LG@MOFs have been proposed as potential candidates for anti-counterfeiting applications as they are capable of encrypting and decrypting the information by changing their luminescent properties. Below we shall consider examples that demonstrate how the use of inks made from luminescent perovskites encapsulated within the UiO-66 and Eu-BTC MOFs are promising materials for anti-counterfeiting protection. The fact that perovskites are very sensitive to external factors (including water), confers them the ability to encrypt the 
information (turning-off the emission) simply through water exposure. ${ }^{120,250}$ This information can be recovered (turning-on the emission) when the material is impregned with a solution of methylammonium bromide (MABr) as shown in Figure 32(a-b). ${ }^{120,250}$

a)

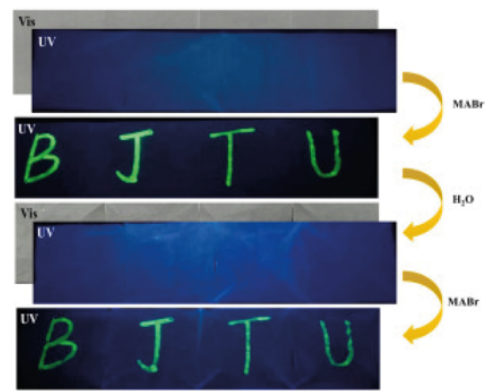

c)

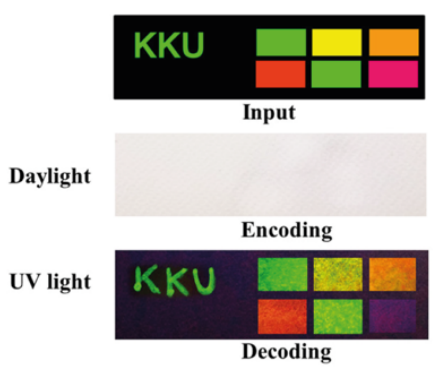

b)

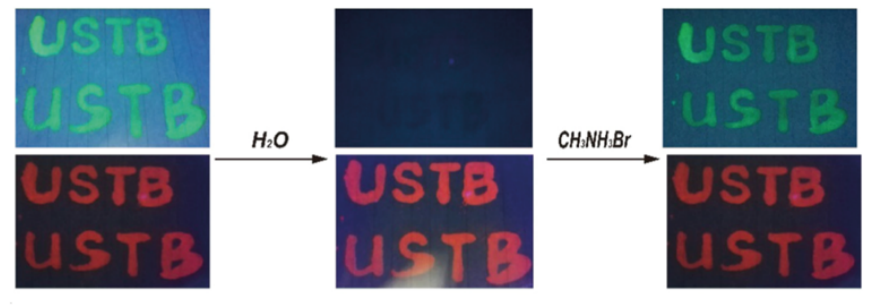

d)

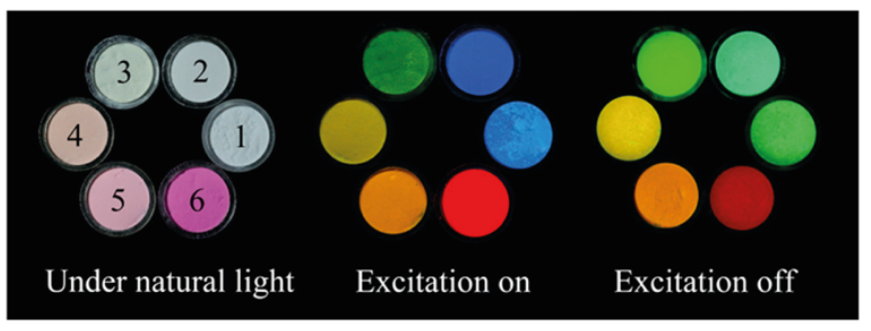

Figure 32. $(a, b)$ Reversible switching of the luminescence of perovskite encapsulated within (a) UiO-66 (Adapted with permission from ref. 250. Copyright 2020 Elsevier) and (b) Eu-BTC MOFs (Adapted with permission from ref. 120. Copyright 2018 American Chemical Society) upon exposure to water and methylammonium bromide. (c) Image of code information (top image) and drawn output under daylight (transparent) and UV irradiation (decoding the information). Adapted with permission from ref. 112. Copyright 2019 American Chemical Society. (d) Photographs under ambient light (left), UV excitation on (middle), and UV excitation off (right) of: 1= Cd(m-bdc)(bIm) MOF; 2 =4-methylumbelliferone@Cd-MOF; 3 =Fluorescent Green B@Cd-MOF; $4=$ Rhodamine 123@Cd-MOF; 5=Rhodamine6G@CdMOF; and 6= Rhodamine B@Cd-MOF. Adapted with permission from ref. 142. Copyright 2018 American Chemical Society.

Another interesting example consisting of carbon dots (CDs) within bimetallic lanthanoids MOFs has displayed the ability for encoding and decoding information by UV light exposure. ${ }^{12}$ Here, the written information was invisible under daylight, but it turns visible under UV light, keeping the color and information very accurate to the expected input Figure 32(c). Additionally, the authors demonstrate that it is possible to write information employing a styrene ink, which quenched the emission due to a photoinduced charge transfer mechanism. Interestingly, the information was erased after 1 hour by a simple evaporation of the styrene, and it can be rewritten again, reducing in this way the waste of paper. ${ }^{12}$ 
A completely different approach for using LG@MOF against anti-counterfeiting was based on materials exhibiting a long-lived luminescence emission. For instance, the encapsulation of different guests (4-methylumbelliferone, Fluorescent Green B, Rhodamine 123, Rhodamine 6G and Rhodamine B) within the long-lived emissive $\mathrm{Cd}(\mathrm{m}-\mathrm{bdc})(\mathrm{bIm}) \mathrm{MOF}$, resulting in multiple composites which exhibit a luminescence that can last for a few seconds (Figure 32d). ${ }^{142}$ Furthermore, an ink containing RhB encapsulated within the $\mathrm{Cd}(\mathrm{m}-\mathrm{bdc})(\mathrm{bIm})$ MOF was used to create a stamp that displays a red fluorescent emission under UV irradiation, but it transforms to a dark orange long-lived emission upon the removal of UV excitation, showing the potential of deploying such a material for anticounterfeiting purposes. ${ }^{142}$

\subsection{Light Emitting Devices (LEDs) and Optical Convertors}

Light emitting diodes (LEDs) have emerged as one of the most efficient devices for light illumination and screens fabrication, reducing the global energy consumption, and therefore contributing to global sustainable growth. LEDs have many advantages over their traditional counterparts, such as the incandescent and halogen bulbs. For instance, the emission of light from LEDs is more directed, which reduce the use of diffusers and reflectors. Additionally, LEDs release $90 \%$ less heat than the incandescent bulbs while their life expectancy is 3 to 25 times longer, and most importantly, LEDs can save energy up to $80 \%$ more efficiently than the incandescent and halogens bulbs. ${ }^{251} \mathrm{Up}$ till now, most of the commercial LEDs are fabricated with rare-earth elements, which are toxic and costly, ${ }^{252,253}$ and thus, there is an urgent need to replace these materials with greener alternatives.

LG@MOF has become a very promising alternative that attracted much attention during the last few years. Most of the examples reported hitherto are down-converter type LEDs, where typically a UV or blue LED is coated with the LG@MOF material to yield multicolor and/or white light emitting LEDs. Many examples can be found in the literature, where the luminescent guests can be of different types including organic fluorophores, perovskites, metal-organic complexes, and carbon dots. ${ }^{42,54,70,75,77,78,112,122,136,254-256}$ Even though this method has some advantages, as its simplicity and rapid production, there exists several drawbacks, like the plausible photodegradation of the materials owing to the high energy irradiation from the blue UV LEDs, which reduces the functional lifetime of the device. Having this in mind, an elegant alternative for the fabrication of MOF-LEDs has recently been demonstrated, which consists of the fabrication of a multilayered device in which the emissive layer is an electroluminescent LED. ${ }^{37,225,257-260}$ This field is still in its infancy, and there exists 
only a few examples of electroluminescent LG@MOF materials. In the first reported example, two organic fluorophores were entrapped in the pores of a Zr-based MOF (Zr-NDC). ${ }^{257}$ In this study, the authors fabricated three electroluminescent devices, in which the electroactive layers were the pristine Zr-NDC MOF, C153@Zr-NDC and DCM@Zr-NDC, respectively. It was demonstrated that the pristine MOF was electroluminescent by itself, and that the incorporation of the organic fluorophores has improved the emission efficiency and enables tuning of the emission color of the LED devices. ${ }^{257}$
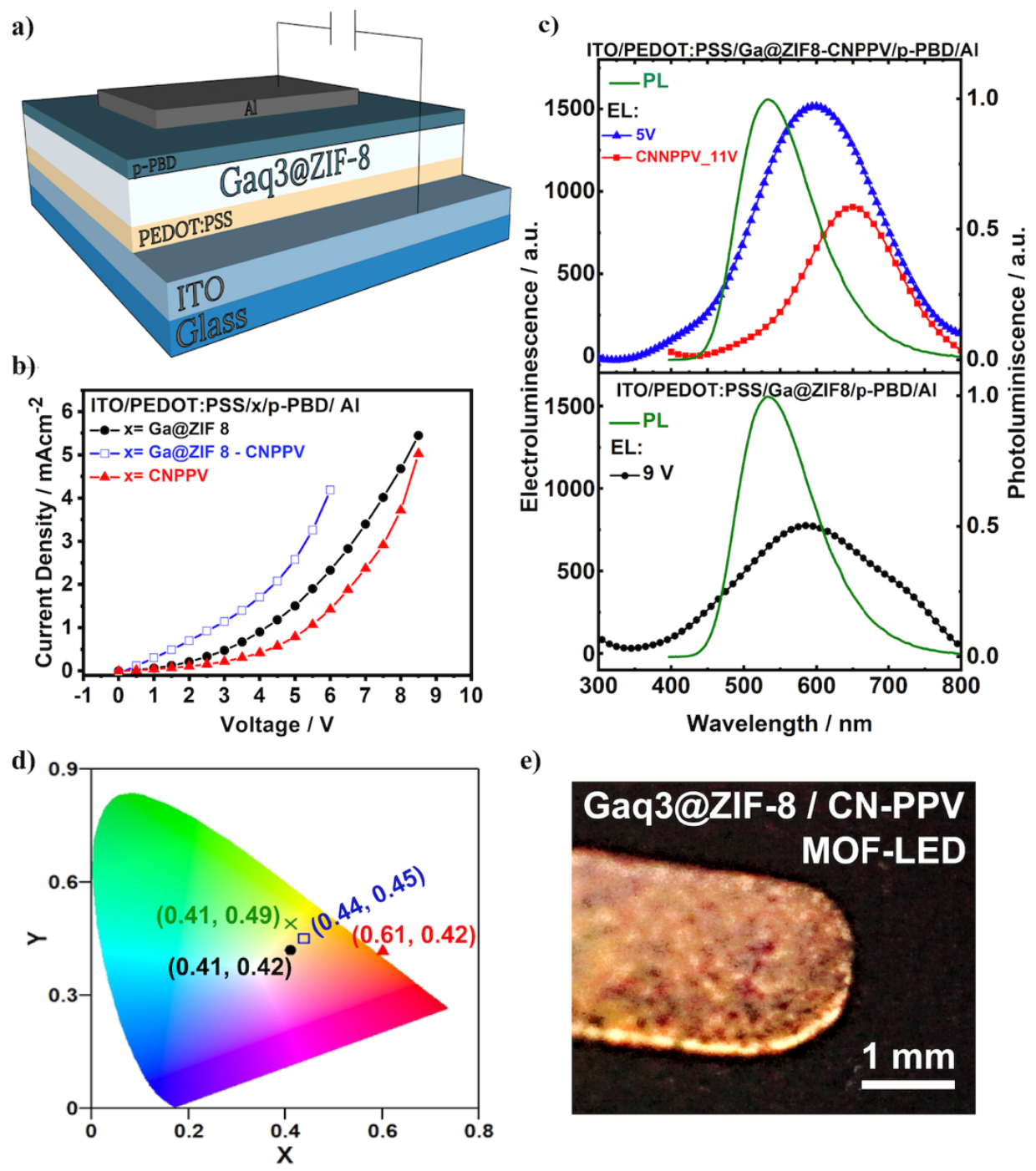

e)

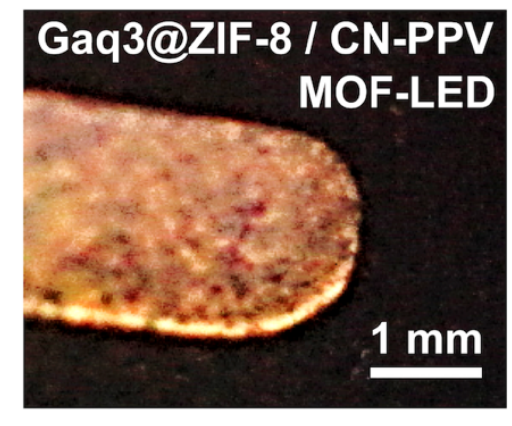

Figure 33. (a) Schematic representation of the multilayer MOF-LED architecture. (b) Current vs voltage curves of the different MOF LEDs, and (c) their corresponding electroluminescent spectra. (d) CIE coordinates corresponding to the photoluminescence of Gaq 3 _ZIF-8 (green cross) and the electroluminescence of the different MOF-LEDs (Gaq 3 @ZIF-8/CNPPV: blue square, and Gaq $@$ @ZIF-8: black dot) and CNPPV-LED (red triangle). (e) Photograph of the MOF-LED device operating at $6 \mathrm{~V}$, in which Gaq 3 QZIF-8 serves as the electroluminescent layer with the characteristic orange-yellowish emission. Adapted from ref. 37. Copyright 2020 John Wiley \& Sons. 
Another possible approach is to incorporate a "non-innocent" guest into the pores of an inert (non-electroluminescent) MOF. Indeed, this is exactly the case of the confinement of the metal complex $\mathrm{Gaq}_{3}$, which is a well-known electroluminescent semiconductor, in the pores of the ZIF-8 host (Figure 33a). ${ }^{37}$ It was demonstrated that the Gaq 3 @ZIF-8 composite functions as the electroactive layer of the MOF-LED device. This material was sandwiched between different polymer layers (electron and hole transport layers), an ITO anode and an Al cathode (Figure 33b). The current vs voltage curves showed that the electroluminescence property was improved when the material was dispersed in CN-PPV [poly(5-(3,7-dimethyloctyloxy-2methoxy-cyanoterephthalydiene], and that the turn-on voltage of the device was relatively low with a value of $\sim 3 \mathrm{~V}$ (Figure 33c). These recent exemplars are just proof-of-concept MOFLED devices, where the efficiency of the devices is still low. Therefore, there is a huge margin for technical improvement, where combined knowledge from chemists, physicist, and engineers could help to boost this interdisciplinary subject.

a)
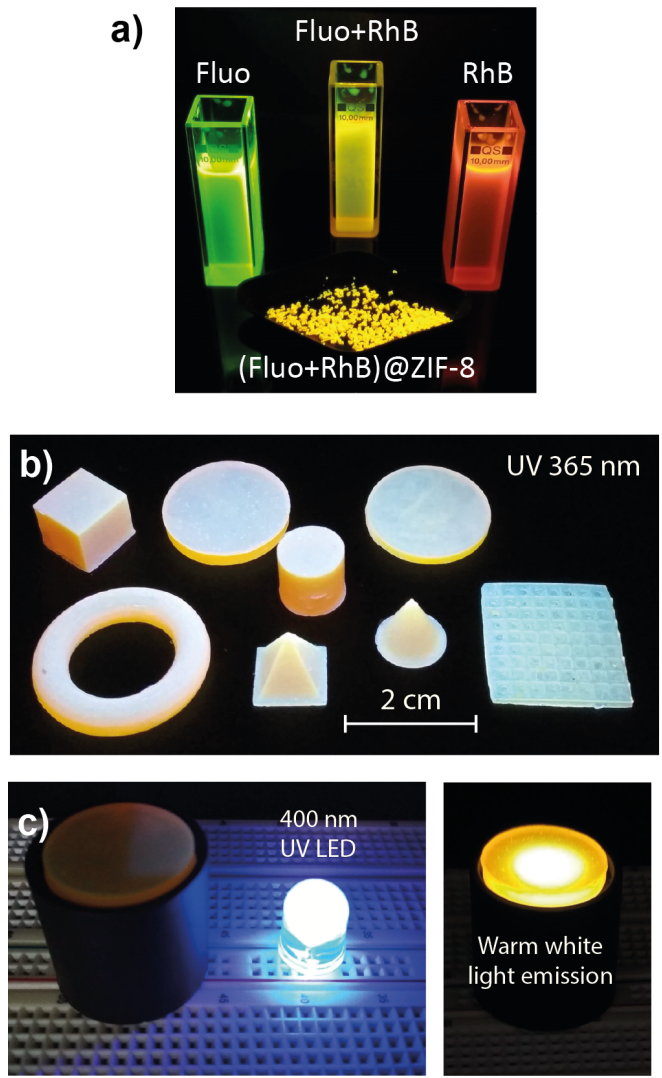
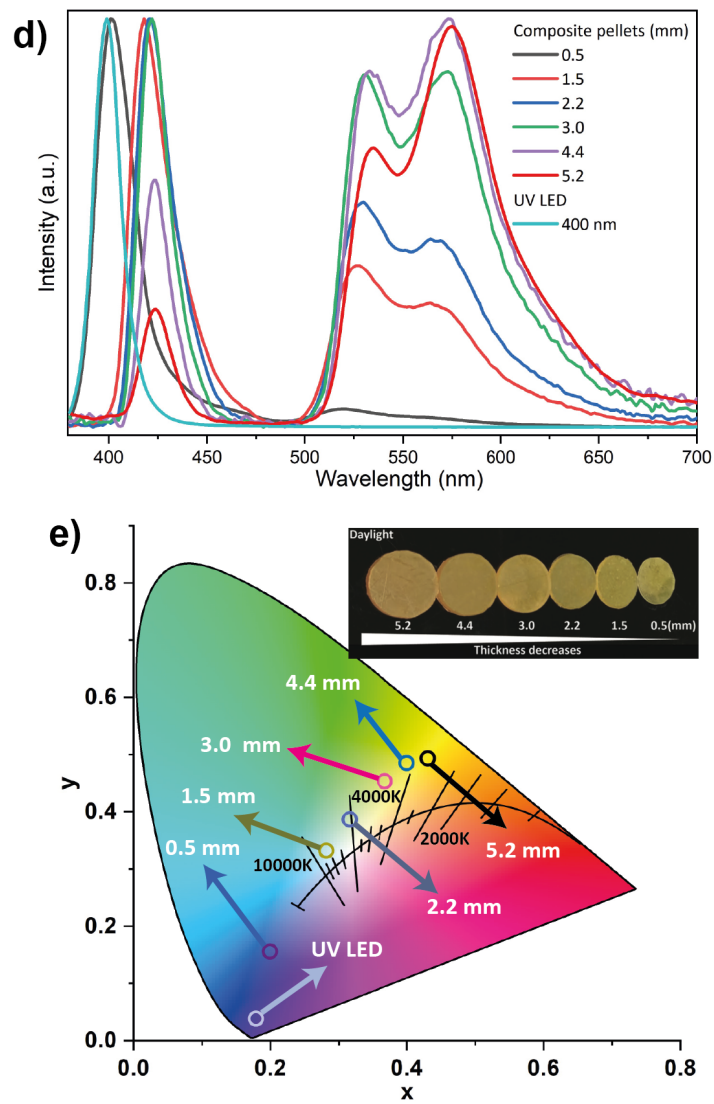

Figure 34. (a) The dual-guest@ZIF8 material encapsulating a combination of fluorescein and rhodamine B dyes to yield a bright yellow emission under 365-nm UV. (b) Various 3D printed geometries viewed under UV. (c) Optical converter disc with a thickness of $3 \mathrm{~mm}$ producing a warm white light when excited by 400-nm UV source in transmission mode. (d, e) Emission spectra and CIE color chromaticity generated by a series of printed discs with a thickness of 
0.5 to $5.2 \mathrm{~mm}$ (inset), tested using the optical conversion setup shown in panel (c). Adapted from ref. 71. Copyright 2020 John Wiley \& Sons.

Finally, we consider a case study on the manufacturing of a LG@MOF optical converters by employing a commercially available 3-D printing methodology. The composite material concerned is the dual-guest encapsulated system, termed (Fluo+RhB)@ZIF-8, which is a bright yellow emitter under UV irradiation (Figure 34a). ${ }^{71}$ The dual-guest nanomaterial was synthesized using the HCR method via a rapid one-pot reaction (Section 2.2), yielding crystals with a 2-D nanodisc morphology with a mean thickness of under $20 \mathrm{~nm}$. The nanocrystals were then combined with a commercial-grade acrylic based photopolymer (a blue emitter under UV), uniformly dispersed to yield a 3-D printable luminescent composite resin for constructing a range of geometries depicted in Figure 34b. For example, a 3-mm thick printed disc $(\Phi=44 \%)$ can be used to build a warm white LED (down-conversion device) excited by a $400 \mathrm{~nm}$ UV source. Furthermore, it was demonstrated that by systematically adjusting the thickness of the 3-D printed discs from $\sim 0.5$ to $5 \mathrm{~mm}$, simple optical convertors can be engineered for tuning the emission color chromaticity starting from a cool white light all the way to a warm color emission (CCT range of 8,300 K to 3,700 K), see Figure 34c. This study suggests that the combination of stereolithography and other related high-resolution 3-D printing methodologies ${ }^{261,262}$ with LG@MOF based nanomaterials could pave the way to the engineering and manufacturing of both precision and bulkier components for real-world applications.

\section{PERSPECTIVES AND OUTLOOK}

Encouragingly, the research on LG@MOF materials and their composite systems is gathering momentum. The literature is expanding swiftly, thus far generating a vast range of enticing photophysical and photochemical properties with interesting possibilities for innovative applications. The nanoconfinement or encapsulation of luminescent guests within a MOF host is a radical shift from the traditional route to yielding LMOF materials with intrinsic luminescence. In this review, we show that the LG@MOF concept is indeed highly versatile. In principle, it confers multiple pathways to design, fabricate, tune, and engineer the guest-host interactions for obtaining luminescent properties targeting bespoke applications, examples of which include intelligent optical sensors and photo switches, energy-efficient lighting, and 
stimuli-responsive photonics. As the number of reported LG@MOF systems and their potential applications continue to grow, it is timely to identify challenges surrounding this nascent field so as to motivate further investigations from both the basic science and practical application standpoints.

(a) Confinement pathways. While general synthetic guidelines do exist to assist with identifying the possible pathways to encapsulate luminescence guests within a MOF host (e.g., in situ vs. post synthetic confinement), the universality of the primary approaches is yet to be proven. The success of a LG@MOF synthesis is often reliant on multiple factors, beyond the straightforward choice of which guest species and compatible host to use. This is probably unsurprising from the perspective of MOF synthesis given the vast range of guest/host combinations which one can theoretically use, however, in practice, certain synthetic constraints do exist for successful fabrication of a desired LG@MOF system. On this matter, sample reproducibility is important and all details about the synthesis method and its scalability (or lack of) should be carefully reported, including caveats that might lead to a failed synthesis for a known system. For instance, thorough washing of the encapsulated sample is often overlooked, which may lead to luminescent guests adhering on the external surfaces and not truly confined inside the MOF structure. Of course, the washing step presents another problem for channel-type MOFs where the leaching of encapsulated guests is likely an issue. In practical terms, another challenge concerns the solvent wastes generated by solution-based synthesis and subsequent washing steps needed for HCR process and liquid impregnation routes. Gasphase loading is solvent-free (guest sublimation and pore insertion) but may require a longer exposure time and specialist equipment (pressure and temperature control), nevertheless, the initial stage where the MOF host is synthesized typically requires the use of solvents. On this basis, the development of a greener synthetic pathway will be attractive, for instance mechanochemistry, ${ }^{263}$ although its general efficacy to confine luminescent guests is still relatively unknown. ${ }^{264}$ And, even if it delivers, this solvent-free approach will likely to be applicable to a specific combination of guest/host only but this is worth exploring.

(b) Fluorescent guest loading. Quantitative determination of the amount of guest present in MOF host is often challenging, therefore this information is not well described or missing from published works. NMR, TGA, EA, UV-Vis methods may be appropriate for estimating the amount of guest loading for a specific LG@MOF system. Connected to point (a) above, to ensure reproducibility the reported values should make clear on how the calculations were 
made to arrive at the guest concentration claimed. However, the above techniques disregard whether the guest molecules are confined in the host structure/pores, or merely adhered on the external surfaces of the MOF parent crystals. The latter case is highly problematic for poorly washed samples, since the surface-bound guest species will inevitably cause an overestimation in quantification of the encapsulated-guest loading. To validate the success of the guest confinement/encapsulation strategy, this will require the use of complementary characterization techniques described in Section 4, especially the application of local-scale methodologies such as nanoFTIR, HRTEM, CLSM, and high-resolution tools available at the synchrotron and neutron facilities (spectroscopy, diffraction, and imaging).

(c) Photodynamics and mechanisms. Time-resolved photophysical measurements of LG@MOF materials is generally lacking in the literature. Whilst excitation-emission spectra are commonplace, detailed photodynamics characterization of emission lifetime of the bulk samples (e.g., TCSPC) and at local scale (e.g., CLSM) are relatively uncommon for all reported LG@MOF systems. By untangling the different components of the multiexponential emission lifetimes this could reveal, for example, the formation of the H- and J-aggregates in addition to identifying contribution of the isolated monomers. Detailed photodynamic characterization could also shed light on unexpected energy transfer phenomenon associated with guest-host coupling, an improved understanding of which may allow one to control the luminescence behavior of LG@MOF and manipulate its response. More broadly, systematic investigations into the guest-host interactions and energy/charge transfer phenomenon are warranted, to help untangle the underpinning mechanisms governing the observed photobehavior. The mechanisms proposed for many of the reported studies concerned LG@MOFs are either vaguely explained or only speculative. The application of theoretical methods such as ab initio density functional theory (DFT) should be encouraged to study LG@MOF systems, where the main challenges lie in the modelling of spatial confinement of a large system with dispersion correction and their electronic structures, and simulation of excited-state events using the timedependent DFT (TDDFT). To this end, the recent development and implementation of computationally efficient yet accurate DFT methods are promising to simulate the structureproperty relationships of large Guest@MOF systems. ${ }^{83,265}$

(d) Long-term materials stability. Improved understanding should be sought about the photostability of LG@MOF systems subject to UV irradiation and other environmental factors. A limited number of accelerated photodegradation studies have suggested a likely 
enhancement in photostability of fluorophores, where a slower degradation rate was recorded upon guest confinement in MOF structure hence increasing their longevity. ${ }^{83}$ More systematic studies are warranted to investigate how the host framework may shield and protect the lightsensitive guest molecules to reduce or impede negative effects of photobleaching. ${ }^{40,266}$ Information on the long-term durability of LG@MOFs under ambient conditions is particularly lacking in literature, to study how the exposure to humidity and ambient temperature might alter material performance over time. For real-world applications, data will be needed to establish the influence of external stimuli (e.g., elevated temperature and pressure, stress, VOCs, $\mathrm{pH}$ ) on sensor calibration and precision if a multiple-use device is needed.

(e) Disruptive sensors for real-time detection. The development of LG@MOF composite systems has led to new materials potentially useful for turn-on luminescent sensors, setting them apart from the conventional class of turn-off type LMOFs. Encouragingly, several candidates for self-calibrating ratiometric sensors have also been demonstrated, where the dualemissive properties may be harnessed to auto correct for external interferences in real time. Moreover, it was recently demonstrated that aggregation of AIE luminogens in MOF cages could yield a rare turn-on response subject to a mechanical stress, which is encouraging for mechanofluorochromic sensing application. ${ }^{240}$ These are highly promising research avenues where LG@MOFs have the edge over conventional systems that rely on intrinsic luminescence, but have not been explored with much depth. Yet another area that warrants further exploration is VOC sensing in vapor phase, which remains challenging for conventional materials but has been shown to be tractable adopting the concept of LG@MOF. ${ }^{35}$ Good selectivity (including water stability and resistance against ambient humidity), rapid response time, reversibility, and a turn-on response are amongst the challenges to be overcome to yield practical VOC vapor sensors. ${ }^{267}$

(f) Electroluminescent devices. In the light of the promising proof-of-concept devices demonstrated so far, ${ }^{37,225,257-260}$ LG@MOF composites have the prospect of becoming an emergent class of photoactive materials to engineer the rare-earth-free and tunable electroluminescent MOF-LEDs. It will be essential to initiate concerted efforts in this central topic, aimed at improving the quantum yield and photostability of the electroactive layers, enhancing the device energy efficiency and increase its overall durability subject to real-world service conditions. Further to addressing the foregoing barriers, innovative advancements in down-stream fabrication of LG@MOF based applications and fine-scale device packaging will 
compel either creative adaptations of existing manufacturing methodologies or new developments of micro-/nanofabrication inspired by latest advancements witnessed in highresolution 3-D printing, precision inkjet printing, electrospinning and lithography technologies that must be compatible with fluorescent guests, organic and inorganic building units.

(g) Mechanical properties. Ultimately, there is the vital question of understanding the science to design and control the mechanical behavior of bespoke systems constituting LG@MOF crystals, polycrystalline films, bulk monoliths and hybrid composites. Central to "MOF mechanics" are a variety of problems encompassing: elasticity and flexibility, timedependent viscous effects (creep and recovery), adhesion of dissimilar structures and debonding of interfaces, dynamics upon impact and mechanical dissipation, crack propagation and fracture. For example, establishing accurate structure-mechanical property relationships of LG@MOFs will be key to the engineering of mechano-sensors whose operation will rely on mechanofluorochromic effects. Recent developments seen in the adaptation of fine-scale mechanical characterization tools for probing MOF films and monoliths going beyond the elastic limit, ${ }^{268-270}$ bode well for the field. Systematic investigations are merited in the broader context of guest-encapsulated framework assemblies, while tackling the farseeing goal for enhancing mechanical resilience of sensors and devices destined for practical use.

In conclusion, the review shows that the fascinating concept of LG@MOF holds a tremendous promise for engineering unconventional fluorescent materials by nanoscale confinement, whose photophysics and photochemistry could yield revolutionary sensors, smart devices and next-generation lighting. The transformative aspects of LG@MOFs are creating a fertile ground perfect for vibrant interdisciplinary research, for which we envisage its growth will continue to accelerate for the next $\sim 10$ years. We are optimistic that the numerous opportunities and challenges identified in this review will further entice and stimulate the multifaceted materials community, including (but not limited to) chemists, engineers, physicists and computational scientists, to innovate and join forces to majorly advance this exciting research. 


\section{AUTHOR INFORMATION}

\section{Corresponding Author:}

Jin-Chong Tan - Multifunctional Materials \& Composites (MMC) Laboratory, Department of Engineering Science, University of Oxford, Parks Road, Oxford OX1 3PJ, United Kingdom. E-mail: jin-chong.tan@eng.ox.ac.uk

\section{Authors:}

Mario Gutiérrez - Multifunctional Materials \& Composites (MMC) Laboratory, Department of Engineering Science, University of Oxford, Parks Road, Oxford OX1 3PJ, United Kingdom.

${ }^{\dagger}$ Current address: Departamento de Química Física, Facultad de Ciencias Ambientales y Bioquímica, INAMOL, Universidad de Castilla-La Mancha, Avenida Carlos III, S/N, 45071 Toledo, Spain.

Yang Zhang - Multifunctional Materials \& Composites (MMC) Laboratory, Department of Engineering Science, University of Oxford, Parks Road, Oxford OX1 3PJ, United Kingdom.

\section{Notes}

The authors declare no competing financial interest.

\section{Biographies}

JCT: Jin-Chong Tan is Professor of Nanoscale Engineering in the Department of Engineering Science at Oxford University. He received his BEng (UTM Malaysia, 1999) and MEng (NTU Singapore, 2001) in mechanical \& materials engineering, and $\mathrm{PhD}$ in materials science (2005) from Cambridge University. In 2012, he founded the MMC Lab at Oxford, focusing on the physical and functional properties of novel materials targeting real-world applications. Recent research interests include nanoengineering of luminescent composites for photonics and sensing technologies.

MG: Mario Gutiérrez obtained his degrees in chemistry (2012), MSc (2014), and Ph.D. in nanoscience and nanotechnology (2017) at the University of Castilla-La Mancha (UCLM). 
Then, he joined the University of Oxford (MMC Laboratory) as a postdoctoral researcher (2019-2020). From 2021, he is an Associate Professor at UCLM (Faculty of Environmental Sciences and Biochemistry). His research interests are focused on the development and photophysical characterization of novel luminescent porous materials (MOFs, COFs and HOFs) for applications in luminescent sensing and LED devices.

YZ: Yang Zhang received his Bachelor's and Master's degree from Northwestern Polytechnical University (China) and Loughborough University (UK), respectively. Currently, he is pursuing a $\mathrm{PhD}$ in Engineering Science under Professor Jin-Chong Tan's guidance in the MMC Laboratory at Oxford. His research is centered on investigating the guest-MOF interactions to yield tunable nanocomposites for emerging optoelectronics and sensing devices.

\section{ACKNOWLEDGMENTS}

J.C.T, M.G. and Y.Z. are grateful to the ERC Consolidator Grant (PROMOFS 771575) for funding the research. M.G. thanks the financial support from the EU through the FSE+. 


\section{ABBREVIATIONS}

AFM

AIE

ANT

ATR

$\mathrm{Au}$

bdc

BI

bIm

BODIPY

BPEI-CDs

BPT

BSE

C153

C343

$\mathrm{C} 460$

C460

CCD

$\mathrm{CCM}$

CDs

CLSM

$\mathrm{CN}^{-}$

CN-PPV

$\mathrm{COK}$

CT

Cyt $c$

DAAC

DASP $^{+}$

DBA

DCM

DFT atomic force microscopy

aggregation induced emission

anthracene

attenuated total reflectance

gold

1,4-benzenedicarboxylate

benzylidene imidazolinone

benzimidazole

boron-dipyrromethene

branched poly-(ethylenimine)-capped carbon quantum dots

biphenyl-3,4',5-tricarboxylate

backscattered electron

coumarin 153

coumarin 343

coumarin 460

coumarin 460

charge coupled device

curcumin

carbon quantum dots

confocal laser scanning microscopy

cyanide ions

poly(5-(3,7-dimethyloctyloxy-2-methoxy-cyanoterephthalydiene

Centrum voor Oppervlaktechemie en Katalyse

charge transfer

cytochrome c

3,6-diaminoacridinium cation

(4-p-(dimethylamino)styryl)-1-methylpyridinium

9,10-dibenzoate anthracene

4-(dicyanomethylene)-2-methyl-6-(4-dimethylaminostyryl)-4H-pyran

density functional theory 
DHT

DMA

DMF

DXP

EA

EDS/EDX/EDXS

ESPT

ESIPT

EXAFS

FIB

FIR-53

Fluo

FRET

FTIR

$\mathrm{Gaq}_{3}$

GSH

$\mathrm{H}_{2}$ TZB

HAADF

HCR

HPTS

HRTEM

HTATAB

ICP-OES

ICT

Ir

K-M

LEDs

LG

LMOF

Ln

$\mathrm{MABr}$

mIm 2,5-dihydroxyterephthalic acid

dimethylacetamide

$\mathrm{N}, \mathrm{N}$-dimethylformamide

N,N-bis(2,6-dimethylphenyl)-3,4:9,10-perylene tetracarboxylic diimide elemental analysis

energy dispersive X-ray spectroscopy

excited state proton transfer

excited state intramolecular proton transfer

extended X-ray absorption fine structure

focused ion beam

$\left.\mathrm{Zn}_{2}(\mathrm{tipa})_{2}\left(\mathrm{OH}^{-}\right)\right]\left(\mathrm{NO}_{3}{ }^{-}\right)(\mathrm{SG} 7)_{2 / 3} \cdot 5 \mathrm{H}_{2} \mathrm{O}$

fluorescein

Förster energy transfer

Fourier transform infrared

gallium(III) tris(8-hydroxyquinolinato)

glutathione

4-(1H-tetrazol-5-yl)benzoic acid

high-angle annular dark-field

high-concentration reaction

8-hydroxy-1,3,6-pyrenetrisulfonicacid trisodium

high-resolution transmission electron microscopy

4,4',4"'-s-triazine-1,3,5-triyltri-p-aminobenzoic acid

inductively coupled plasma optical emission spectroscopy

intramolecular charge transfer

iridium

Kubelka-Munk

light emitting diodes

luminescent guest

luminescent MOF

lanthanide

methylammonium bromide

2-methylimidazole 
MOF

MOL

nanoFTIR

$\mathrm{NCs}$

NDC

NKU

nMOFs

NMR

$\mathrm{NO}_{2}$-bdc

NR

NTB

o-phen

O.D.

OLEDs

OX

PCT

PEG-ZnS

PONs

PTA

PXRD

q

R6G

Rh101

Rh6G

$\mathrm{RhB}$

Rs

Rubpy

s-SNOM

SBE

SBMs

$\mathrm{SC}$

SCXRD metal-organic framework

metal-organic layer

nanoscale Fourier transform infrared spectroscopy

nanocrystals

2,6-naphthalenedicarboxylate

Nankai University

nanometric MOFs

nuclear magnetic resonance

2-nitro-4-benzenedicarboxylicacid

Nile red

4,4',4"'-nitrilotrisbenzoic acid

1,10-phenanthroline

optical density

organic light-emitting diodes

University of Oxford material

photoinduced charge transfer

polyethylene glycol-capped $\mathrm{ZnS}$

porous organic nanosheets

p-phthalic acid

powder X-ray diffraction

8-hydroxyquinoline

rhodamine $6 \mathrm{G}$

rhodamine 101

rhodamine $6 \mathrm{G}$

rhodamine $\mathrm{B}$

resorufin

$\mathrm{Ru}(\mathrm{II})$ tris(2,2'-bipyridine)

scattering-type scanning near-field optical microscopy

back-scattered electrons

silica-based mesoporous systems

single crystal

single crystal X-ray diffraction 
secondary electrons

SEM

scanning electron microscopy

$\mathrm{SG} 7^{3-}$

solvent green 7

SR-IR

synchrotron-radiation infrared

STXM

scanning tunnelling X-ray microscopy

TCPP

5,10,15,20-tetra(carboxyphenyl)porphyrin

TCSPC

time-correlated single photon counting

TDDFT

time-dependent density functional theory

TEA

triethylamine

TEM

transmission electron microscopy

TGA

thermogravimetric analysis

TIPA

tri(4-imidazolylphenyl)amine

TNP

2,4,6-trinitrophenol

TPE

1,1,2,2-tetraphenylethylene

tpt

2,4,6-tri(4-pyridyl)-1,3,5-triazine

$\mathrm{UiO}$

Universitetet i Oslo

UMCM

University of Michigan Crystalline Material

UV

ultraviolet

VOCs

volatile organic compounds

VTGs

VOCs, toxic chemicals and gases

XANES

X-ray absorption near edge structure

XAS

X-ray absorption spectroscopy

XPS

X-ray photoelectron spectroscopy

XRF

$\mathrm{X}$-ray fluorescence

ZIF

zeolitic imidazolate framework

$\mathrm{ZnQ}$

zinc(II) bis-(-8-hydroxyquinoline)

$\Phi$

quantum yield 


\section{REFERENCES}

(1) Freund, R.; Canossa, S.; Cohen, S. M.; Yan, W.; Deng, H.; Guillerm, V.; Eddaoudi, M.; Madden, D. G.; Fairen-Jimenez, D.; Lyu, H.; et al. 25 years of reticular chemistry. Angew. Chem. Int. Ed. 2021, 60, 23946-23974.

(2) O'Keeffe, M.; Yaghi, O. M. Deconstructing the crystal structures of metal-organic frameworks and related materials into their underlying nets. Chem. Rev. 2012, 112, 675-702.

(3) Furukawa, H.; Cordova, K. E.; O'Keeffe, M.; Yaghi, O. M. The chemistry and applications of metal-organic frameworks. Science 2013, 341, 1230444.

(4) Tan, J. C.; Civalleri, B. Metal-organic frameworks and hybrid materials: From fundamentals to applications. CrystEngComm 2015, 17, 197-198.

(5) Farha, O. K.; Eryazici, I.; Jeong, N. C.; Hauser, B. G.; Wilmer, C. E.; Sarjeant, A. A.; Snurr, R. Q.; Nguyen, S. T.; Yazaydin, A. O.; Hupp, J. T. Metal-organic framework materials with ultrahigh surface areas: Is the sky the limit? J. Am. Chem. Soc. 2012, 134, 15016-15021.

(6) Allendorf, M. D.; Foster, M. E.; Leonard, F.; Stavila, V.; Feng, P. L.; Doty, F. P.; Leong, K.; Ma, E. Y.; Johnston, S. R.; Talin, A. A. Guest-induced emergent properties in metal-organic frameworks. J. Phys. Chem. Lett. 2015, 6, 1182-1195.

(7) Allendorf, M. D.; Bauer, C. A.; Bhakta, R. K.; Houk, R. J. T. Luminescent metalorganic frameworks. Chem. Soc. Rev. 2009, 38, 1330-1352.

(8) Lustig, W. P.; Mukherjee, S.; Rudd, N. D.; Desai, A. V.; Li, J.; Ghosh, S. K. Metalorganic frameworks: Functional luminescent and photonic materials for sensing applications. Chem. Soc. Rev. 2017, 46, 3242-3285.

(9) Zhao, Y.; Zeng, H.; Zhu, X. W.; Lu, W.; Li, D. Metal-organic frameworks as photoluminescent biosensing platforms: Mechanisms and applications. Chem. Soc. Rev. 2021, 50, 4484-4513.

(10) Diamantis, S. A.; Margariti, A.; Pournara, A. D.; Papaefstathiou, G. S.; Manos, M. J.; Lazarides, T. Luminescent metal-organic frameworks as chemical sensors: Common pitfalls and proposed best practices. Inorg. Chem. Front. 2018, 5, 1493-1511.

(11) Hao, J.; Xu, X.; Fei, H.; Li, L.; Yan, B. Functionalization of metal-organic frameworks for photoactive materials. Adv. Mater. 2018, 30, e1705634.

(12) Rocha, J.; Brites, C. D.; Carlos, L. D. Lanthanide organic framework luminescent thermometers. Chem. 2016, 22, 14782-14795.

(13) Cheng, T.; Hu, J.; Zhou, C.; Wang, Y.; Zhang, M. Luminescent metal-organic frameworks for nitro explosives detection. Sci. China Chem. 2016, 59, 929-947.

(14) Kumar, P.; Singh, S.; Gupta, B. K. Future prospects of luminescent nanomaterial based security inks: From synthesis to anti-counterfeiting applications. Nanoscale 2016, 8, 14297-14340.

(15) Yin, H. Q.; Yin, X. B. Metal-organic frameworks with multiple luminescence emissions: Designs and applications. Acc. Chem. Res. 2020, 53, 485-495.

(16) Kaur, H.; Sundriyal, S.; Pachauri, V.; Ingebrandt, S.; Kim, K. H.; Sharma, A. L.; Deep, A. Luminescent metal-organic frameworks and their composites: Potential future materials for organic light emitting displays. Coord. Chem. Rev. 2019, 401, 213077.

(17) Lustig, W. P.; Li, J. Luminescent metal-organic frameworks and coordination polymers as alternative phosphors for energy efficient lighting devices. Coord. Chem. Rev. 2018, $373,116-147$. 
(18) Dong, J.; Zhao, D.; Lu, Y.; Sun, W. Y. Photoluminescent metal-organic frameworks and their application for sensing biomolecules. J. Mater. Chem. A 2019, 7, 2274422767.

(19) Zhang, Y.; Yuan, S.; Day, G.; Wang, X.; Yang, X.; Zhou, H.-C. Luminescent sensors based on metal-organic frameworks. Coord. Chem. Rev. 2018, 354, 28-45.

(20) Stock, N.; Biswas, S. Synthesis of metal-organic frameworks (MOFs): Routes to various MOF topologies, morphologies, and composites. Chem. Rev. 2012, 112, 933 969.

(21) Yuan, S.; Feng, L.; Wang, K.; Pang, J.; Bosch, M.; Lollar, C.; Sun, Y.; Qin, J.; Yang, X.; Zhang, P.; et al. Stable metal-organic frameworks: Design, synthesis, and applications. Adv. Mater. 2018, 30, 1704303.

(22) Lu, W.; Wei, Z.; Gu, Z. Y.; Liu, T. F.; Park, J.; Park, J.; Tian, J.; Zhang, M.; Zhang, Q.; Gentle, T.; et al. Tuning the structure and function of metal-organic frameworks via linker design. Chem. Soc. Rev. 2014, 43, 5561-5593.

(23) Zhang, J. P.; Zhang, Y. B.; Lin, J. B.; Chen, X. M. Metal azolate frameworks: From crystal engineering to functional materials. Chem. Rev. 2012, 112, 1001-1033.

(24) Carne, A.; Carbonell, C.; Imaz, I.; Maspoch, D. Nanoscale metal-organic materials. Chem. Soc. Rev. 2011, 40, 291-305.

(25) Juan-Alcañiz, J.; Gascon, J.; Kapteijn, F. Metal-organic frameworks as scaffolds for the encapsulation of active species: State of the art and future perspectives. J. Mater. Chem. 2012, 22, 10102-10118.

(26) Mollick, S.; Mandal, T. N.; Jana, A.; Fajal, S.; Desai, A. V.; Ghosh, S. K. Ultrastable luminescent hybrid bromide perovskite@MOF nanocomposites for the degradation of organic pollutants in water. ACS Appl. Nano Mater. 2019, 2, 1333-1340.

Hester, P.; Xu, S.; Liang, W.; Al-Janabi, N.; Vakili, R.; Hill, P.; Muryn, C. A.; Chen, X.; Martin, P. A.; Fan, X. On thermal stability and catalytic reactivity of Zr-based metal-organic framework (UiO-67) encapsulated Pt catalysts. J. Catal. 2016, 340, 8594.

(28) Hu, X. L.; Qin, C.; Wang, X. L.; Shao, K. Z.; Su, Z. M. A luminescent dye@MOF as a dual-emitting platform for sensing explosives. Chem. Commun. 2015, 51, 1752117524.

(29) Fu, H.-R.; Yan, L.-B.; Wu, N.-T.; Ma, L.-F.; Zang, S.-Q. Dual-emission MOFつdye sensor for ratiometric fluorescence recognition of $\mathrm{rdx}$ and detection of a broad class of nitro-compounds. J. Mater. Chem. A 2018, 6, 9183-9191.

(30) Muller, M.; Devaux, A.; Yang, C. H.; De Cola, L.; Fischer, R. A. Highly emissive metal-organic framework composites by host-guest chemistry. Photochem. Photobiol. Sci. 2010, 9, 846-853.

(31) Tu, M.; Reinsch, H.; Rodriguez-Hermida, S.; Verbeke, R.; Stassin, T.; Egger, W.; Dickmann, M.; Dieu, B.; Hofkens, J.; Vankelecom, I. F. J.; et al. Reversible optical writing and data storage in an anthracene-loaded metal-organic framework. Angew. Chem. Int. Ed. 2019, 58, 2423-2427.

(32) Chaudhari, A. K.; Ryder, M. R.; Tan, J.-C. Photonic hybrid crystals constructed from in situ host-guest nanoconfinement of a light-emitting complex in metal-organic framework pores. Nanoscale 2016, 8, 6851-6859.

(33) Zhang, D. S.; Gao, Q.; Chang, Z.; Liu, X. T.; Zhao, B.; Xuan, Z. H.; Hu, T. L.; Zhang, Y. H.; Zhu, J.; Bu, X. H. Rational construction of highly tunable donor-acceptor materials based on a crystalline host-guest platform. Adv. Mater. 2018, 30, 1804715.

(34) Asadi, F.; Azizi, S. N.; Chaichi, M. J. Green synthesis of fluorescent PEG-ZnS QDs encapsulated into Co-MOFs as an effective sensor for ultrasensitive detection of copper ions in tap water. Mater. Sci. Eng. C Mater. Biol. Appl. 2019, 105, 110058. 
(35) Chaudhari, A. K.; Kim, H. J.; Han, I.; Tan, J. C. Optochemically responsive 2D nanosheets of a 3D metal-organic framework material. Adv. Mater. 2017, 29, 1701463.

(36) Zhang, Y.; Tan, J. C. Electrospun rhodamine@MOF/polymer luminescent fibers with a quantum yield of over 90\%. iScience 2021, 24, 103035 .

(37) Gutiérrez, M.; Martín, C.; Van der Auweraer, M.; Hofkens, J.; Tan, J. C. Electroluminescent guest@MOF nanoparticles for thin film optoelectronics and solidstate lighting. Adv. Opt. Mater. 2020, 8, 2000670.

(38) Chaudhari, A. K.; Tan, J. C. Mechanochromic MOF nanoplates: Spatial molecular isolation of light-emitting guests in a sodalite framework structure. Nanoscale 2018, 10, 3953-3960.

(39) Chin, M.; Cisneros, C.; Araiza, S. M.; Vargas, K. M.; Ishihara, K. M.; Tian, F. Rhodamine B degradation by nanosized zeolitic imidazolate framework-8 (ZIF-8). RSC Adv. 2018, 8, 26987-26997.

(40) Mitsui, M.; Higashi, K.; Takahashi, R.; Hirumi, Y.; Kobayashi, K. Enhanced photostability of an anthracene-based dye due to supramolecular encapsulation: A new type of photostable fluorophore for single-molecule study. Photochem. Photobiol. Sci. 2014, 13, 1130-1136.

(41) Chaudhari, A. K.; Souza, B. E.; Tan, J.-C. Electrochromic thin films of Zn-based MOF74 nanocrystals facilely grown on flexible conducting substrates at room temperature. APL Mater. 2019, 7, 081101.

(42) Chen, W.; Zhuang, Y.; Wang, L.; Lv, Y.; Liu, J.; Zhou, T. L.; Xie, R. J. Color-tunable and high-efficiency dye-encapsulated metal-organic framework composites used for smart white-light-emitting diodes. ACS Appl. Mater. Interfaces 2018, 10, 18910-18917.

(43) Zhang, Y.; Gutierrez, M.; Chaudhari, A. K.; Tan, J. C. Dye-encapsulated zeolitic imidazolate framework (ZIF-71) for fluorochromic sensing of pressure, temperature, and volatile solvents. ACS Appl. Mater. Interfaces 2020, 12, 37477-37488.

(44) Lavis, L. D.; Raines, R. T. Bright ideas for chemical biology. ACS Chem. Biol. 2008, 3, 142-155.

(45) Fu, Y.; Finney, N. S. Small-molecule fluorescent probes and their design. RSC Adv. 2018, 8, 29051-29061.

(46) Yan, B. Lanthanide-functionalized metal-organic framework hybrid systems to create multiple luminescent centers for chemical sensing. Acc. Chem. Res. 2017, 50, 27892798.

(47) Heine, J.; Muller-Buschbaum, K. Engineering metal-based luminescence in coordination polymers and metal-organic frameworks. Chem. Soc. Rev. 2013, 42, 92329242.

(48) An, J. Y.; Shade, C. M.; Chengelis-Czegan, D. A.; Petoud, S.; Rosi, N. L. Zincadeninate metal-organic framework for aqueous encapsulation and sensitization of near-infrared and visible emitting lanthanide cations. J. Am. Chem. Soc. 2011, 133, 1220-1223.

(49) Zhou, Y.; Yan, B. A responsive MOF nanocomposite for decoding volatile organic compounds. Chem. Commun. 2016, 52, 2265-2268.

(50) Liang, Y.-Y.; Luo, L.-J.; Li, Y.; Ling, B.-K.; Chen, B.-W.; Wang, X.-W.; Luan, T.-G. A luminescent probe for highly selective $\mathrm{Cu}^{2+}$ sensing using a lanthanide-doped metal organic framework with large pores. Eur. J. Inorg. Chem. 2019, 2019, 206-211.

(51) Qiu, L.; Yu, C.; Wang, X.; Xie, Y.; Kirillov, A. M.; Huang, W.; Li, J.; Gao, P.; Wu, T.; $\mathrm{Gu}, \mathrm{X}$.; et al. Tuning the solid-state white light emission of postsynthetic lanthanideencapsulated double-layer MOFs for three-color luminescent thermometry applications. Inorg. Chem. 2019, 58, 4524-4533. 
(52) Ji, G.; Zheng, T.; Gao, X.; Liu, Z. A highly selective turn-on luminescent logic gates probe based on post-synthetic MOF for aspartic acid detection. Sens. Actuator B-Chem. 2019, 284, 91-95.

(53) Saines, P. J.; Steinmann, M.; Tan, J. C.; Yeung, H. H. M.; Cheetham, A. K. Structural diversity and luminescent properties of lanthanide 2,2- and 2,3-dimethylsuccinate frameworks. CrystEngComm 2013, 15, 100-110.

(54) Xie, W.; Qin, J.-S.; He, W.-W.; Shao, K.-Z.; Su, Z.-M.; Du, D.-Y.; Li, S.-L.; Lan, Y.Q. Encapsulation of an iridium complex in a metal-organic framework to give a composite with efficient white light emission. Inorg. Chem. Front. 2017, 4, 547-552.

(55) Sun, C. Y.; Wang, X. L.; Zhang, X.; Qin, C.; Li, P.; Su, Z. M.; Zhu, D. X.; Shan, G. G.; Shao, K. Z.; Wu, H.; et al. Efficient and tunable white-light emission of metal-organic frameworks by iridium-complex encapsulation. Nat. Commun. 2013, 4, 2717.

(56) Burrows, P. E.; Sapochak, L. S.; McCarty, D. M.; Forrest, S. R.; Thompson, M. E. Metal ion dependent luminescence effects in metal tris-quinolate organic heterojunction light emitting devices. Appl. Phys. Lett. 1994, 64, 2718-2720.

(57) Jin, R. Quantum sized, thiolate-protected gold nanoclusters. Nanoscale 2010, 2, 343362.

(58) Goswami, N.; Lin, F.; Liu, Y.; Leong, D. T.; Xie, J. Highly luminescent thiolated gold nanoclusters impregnated in nanogel. Chem. Mater. 2016, 28, 4009-4016.

(59) Luo, Z.; Yuan, X.; Yu, Y.; Zhang, Q.; Leong, D. T.; Lee, J. Y.; Xie, J. From aggregation-induced emission of $\mathrm{Au}(\mathrm{I})$-thiolate complexes to ultrabright $\mathrm{Au}(0) @ \mathrm{Au}(\mathrm{I})-$ thiolate core-shell nanoclusters. J. Am. Chem. Soc. 2012, 134, 1666216670.

(60) Tian, R.; Zhang, S.; Li, M.; Zhou, Y.; Lu, B.; Yan, D.; Wei, M.; Evans, D. G.; Duan, $\mathrm{X}$. Localization of $\mathrm{Au}$ nanoclusters on layered double hydroxides nanosheets: Confinement-induced emission enhancement and temperature-responsive luminescence. Adv. Funct. Mater. 2015, 25, 5006-5015.

(61) Pyo, K.; Thanthirige, V. D.; Kwak, K.; Pandurangan, P.; Ramakrishna, G.; Lee, D. Ultrabright luminescence from gold nanoclusters: Rigidifying the Au(I)-thiolate shell. J. Am. Chem. Soc. 2015, 137, 8244-8250.

(62) Coutino-Gonzalez, E.; Baekelant, W.; Steele, J. A.; Kim, C. W.; Roeffaers, M. B. J.; Hofkens, J. Silver clusters in zeolites: From self-assembly to ground-breaking luminescent properties. Acc. Chem. Res. 2017, 50, 2353-2361.

(63) Gao, Q.; Xu, S.; Guo, C.; Chen, Y.; Wang, L. Embedding nanocluster in MOF via crystalline ion-triggered growth strategy for improved emission and selective sensing. ACS Appl. Mater. Interfaces 2018, 10, 16059-16065.

(64) Han, B.; Hu, X.; Yu, M.; Peng, T.; Li, Y.; He, G. One-pot synthesis of enhanced fluorescent copper nanoclusters encapsulated in metal-organic frameworks. RSC $A d v$. 2018, 8, 22748-22754.

(65) Houk, R. J.; Jacobs, B. W.; El Gabaly, F.; Chang, N. N.; Talin, A. A.; Graham, D. D.; House, S. D.; Robertson, I. M.; Allendorf, M. D. Silver cluster formation, dynamics, and chemistry in metal-organic frameworks. Nano Lett. 2009, 9, 3413-3418.

(66) Aguilera-Sigalat, J.; Bradshaw, D. Synthesis and applications of metal-organic framework-quantum dot (QD@MOF) composites. Coord. Chem. Rev. 2016, 307, 267291.

(67) Wang, Y.; Wang, B.; Shi, H.; Zhang, C.; Tao, C.; Li, J. Carbon nanodots in ZIF-8: Synthesis, tunable luminescence and temperature sensing. Inorg. Chem. Front. 2018, 5, 2739-2745. 
(68) Lin, X.; Gao, G.; Zheng, L.; Chi, Y.; Chen, G. Encapsulation of strongly fluorescent carbon quantum dots in metal-organic frameworks for enhancing chemical sensing. Anal. Chem. 2014, 86, 1223-1228.

(69) Mazumdar, S.; Zhao, Y.; Zhang, X. Stability of perovskite solar cells: Degradation mechanisms and remedies. Front. Electron. 2021, 2, 712785.

(70) Zhang, D.; Zhao, J.; Liu, Q.; Xia, Z. Synthesis and luminescence properties of CsPbX 3 @UiO-67 composites toward stable photoluminescence convertors. Inorg. Chem. 2019, 58, 1690-1696.

(71) Chaudhari, A. K.; Tan, J. C. Dual-guest functionalized zeolitic imidazolate framework8 for 3D printing white light-emitting composites. Adv. Opt. Mater. 2020, 8, 1901912.

(72) Wang, B.-H.; Yan, B. A dye@MOF crystalline probe serving as a platform for ratiometric sensing of trichloroacetic acid (TCA), a carcinogen metabolite in human urine. CrystEngComm 2019, 21, 4637-4643.

(73) Zhang, N.; Zhang, D.; Zhao, J.; Xia, Z. Fabrication of a dual-emitting dye-encapsulated metal-organic framework as a stable fluorescent sensor for metal ion detection. Dalton Trans. 2019, 48, 6794-6799.

(74) Liu, X.-Y.; Xing, K.; Li, Y.; Tsung, C.-K.; Li, J. Three models to encapsulate multicomponent dyes into nanocrystal pores: A new strategy for generating highquality white light. J. Am. Chem. Soc. 2019, 141, 14807-14813.

(75) Cui, Y. J.; Song, T.; Yu, J. C.; Yang, Y.; Wang, Z. Y.; Qian, G. D. Dye encapsulated metal-organic framework for warm-white LED with high color-rendering index. $A d v$. Funct. Mater. 2015, 25, 4796-4802.

(76) Xia, T.; Song, T.; Cui, Y.; Yang, Y.; Qian, G. A dye encapsulated terbium-based metalorganic framework for ratiometric temperature sensing. Dalton Trans. 2016, 45, 1868918695.

(77) Song, T.; Zhang, G.; Cui, Y.; Yang, Y.; Qian, G. Encapsulation of coumarin dye within lanthanide MOFs as highly efficient white-light-emitting phosphors for white LEDs. CrystEngComm 2016, 18, 8366-8371.

(78) Wen, Y.; Sheng, T.; Zhu, X.; Zhuo, C.; Su, S.; Li, H.; Hu, S.; Zhu, Q. L.; Wu, X. Introduction of red-green-blue fluorescent dyes into a metal-organic framework for tunable white light emission. Adv. Mater. 2017, 29, 1700778.

(79) Ryu, U.; Yoo, J.; Kwon, W.; Choi, K. M. Tailoring nanocrystalline metal-organic frameworks as fluorescent dye carriers for bioimaging. Inorg. Chem. 2017, 56, 1285912865.

(80) Yoo, J.; Ryu, U.; Kwon, W.; Choi, K. M. A multi-dye containing MOF for the ratiometric detection and simultaneous removal of $\mathrm{Cr}_{2} \mathrm{O}_{7}{ }^{2-}$ in the presence of interfering ions. Sens. Actuator B-Chem. 2019, 283, 426-433.

(81) Liu, X.-Y.; Li, Y.; Tsung, C.-K.; Li, J. Encapsulation of yellow phosphors into nanocrystalline metal-organic frameworks for blue-excitable white light emission. Chem. Commun. 2019, 55, 10669-10672.

(82) Zhang, Y.-Q.; Wu, X.-H.; Mao, S.; Tao, W.-Q.; Li, Z. Highly luminescent sensing for nitrofurans and tetracyclines in water based on zeolitic imidazolate framework-8 incorporated with dyes. Talanta 2019, 204, 344-352.

(83) Xiong, T.; Zhang, Y.; Donà, L.; Gutiérrez, M.; Möslein, A. F.; Babal, A. S.; Amin, N.; Civalleri, B.; Tan, J.-C. Tunable fluorescein-encapsulated zeolitic imidazolate framework-8 nanoparticles for solid-state lighting. ACS Appl. Nano Mater. 2021, 4, 10321-10333.

(84) Haldar, R.; Matsuda, R.; Kitagawa, S.; George, S. J.; Maji, T. K. Amine-responsive adaptable nanospaces: Fluorescent porous coordination polymer for molecular recognition. Angew. Chem. Int. Ed. 2014, 53, 11772-11777. 
(85) Gutiérrez, M.; Sánchez, F.; Douhal, A. Efficient multicolor and white light emission from Zr-based MOF composites: Spectral and dynamic properties. J. Mater. Chem. B 2015, 3, 11300-11310.

(86) Dong, M. J.; Zhao, M.; Ou, S.; Zou, C.; Wu, C. D. A luminescent dye@MOF platform: Emission fingerprint relationships of volatile organic molecules. Angew. Chem. Int. Ed. 2014, 53, 1575-1579.

(87) Wang, Z.; Liu, Y.; Wang, Z.; Cao, L.; Zhao, Y.; Wang, C.; Lin, W. Through-space förster-type energy transfer in isostructural zirconium and hafnium-based metalorganic layers. Chem. Commun. 2017, 53, 9356-9359.

(88) Xia, Y.-P.; Wang, C.-X.; An, L.-C.; Zhang, D.-S.; Hu, T.-L.; Xu, J.; Chang, Z.; Bu, X.H. Utilizing an effective framework to dye energy transfer in a carbazole-based metalorganic framework for high performance white light emission tuning. Inorg. Chem. Front. 2018, 5, 2868-2874.

(89) Holder, C. F.; Schaak, R. E. Tutorial on powder X-ray diffraction for characterizing nanoscale materials. ACS Nano 2019, 13, 7359-7365.

(90) Fu, H.-R.; Wu, X.-X.; Ma, L.-F.; Wang, F.; Zhang, J. Dual-emission SG7@MOF sensor via SC-SC transformation: Enhancing the formation of excimer emission and the range and sensitivity of detection. ACS Appl. Mater. Interfaces 2018, 10, 1801218020.

(91) Wang, C.; Tian, L.; Zhu, W.; Wang, S.; Wang, P.; Liang, Y.; Zhang, W.; Zhao, H.; Li, G. Dye@bio-MOF-1 composite as a dual-emitting platform for enhanced detection of a wide range of explosive molecules. ACS Appl. Mater. Interfaces 2017, 9, 2007620085.

(92) Alarcos, N.; Cohen, B.; Ziółek, M.; Douhal, A. Photochemistry and photophysics in silica-based materials: Ultrafast and single molecule spectroscopy observation. Chem. Rev. 2017, 117, 13639-13720.

(93) Zhao, L.-M.; Shen, X.-Q.; Tan, L.-T.; Zhang, W.-T.; Song, K.-Y.; Jiang, R.; Li, H.-H.; Chen, Z.-R. Encapsulation of stilbazolium-type dyes into layered metal-organic frameworks: Solvent-dependent luminescence chromisms and their mechanisms. Inorg. Chem. Front. 2019, 6, 1195-1208.

(94) Let, S.; Samanta, P.; Dutta, S.; Ghosh, S. K. A dye@MOF composite as luminescent sensory material for selective and sensitive recognition of Fe(III) ions in water. Inorg. Chim. Acta 2020, 500, 119205.

(95) Hamilton, G.; Sanabria, H. of Chapter 6: Multiparameter fluorescence spectroscopy of single molecules. In Spectroscopy and dynamics of single molecules; Johnson, C. K., Ed.; Elsevier, 2019; pp 269-333.

(96) Dolgopolova, E. A.; Williams, D. E.; Greytak, A. B.; Rice, A. M.; Smith, M. D.; Krause, J. A.; Shustova, N. B. A bio-inspired approach for chromophore communication: Ligand-to-ligand and host-to-guest energy transfer in hybrid crystalline scaffolds. Angew. Chem. Int. Ed. 2015, 54, 13639-13643.

(97) Wang, Z.; Wang, Z.; Lin, B.; Hu, X.; Wei, Y.; Zhang, C.; An, B.; Wang, C.; Lin, W. Warm-white-light-emitting diode based on a dye-loaded metal-organic framework for fast white-light communication. ACS Appl. Mater. Interfaces 2017, 9, 35253-35259.

(98) Haldar, R.; Prasad, K.; Hazra, A.; Maji, T. K. Adaptive and guest responsive supramolecular porous framework: Solvent modulated energy transfer toward fingerprint sensing. Cryst. Growth Des. 2019, 19, 1514-1517.

(99) Fu, H.-R.; Zhao, Y.; Xie, T.; Han, M.-L.; Ma, L.-F.; Zang, S.-Q. Stable dyeencapsulated indium-organic framework as dual-emitting sensor for the detection of $\mathrm{Hg}^{2+} / \mathrm{Cr}_{2} \mathrm{O}_{7}{ }^{2-}$ and a wide range of nitro-compounds. J. Mater. Chem. B 2018, 6, 64406448 . 
(100) Yang, L.; Liu, Y.-L.; Liu, C.-G.; Ye, F.; Fu, Y. Two luminescent dye@MOFs systems as dual-emitting platforms for efficient pesticides detection. J. Hazard. Mater. 2020, 381, 120966.

(101) Li, Z.; Wang, G.; Ye, Y.; Li, B.; Li, H.; Chen, B. Loading photochromic molecules into a luminescent metal-organic framework for information anticounterfeiting. Angew. Chem. Int. Ed. 2019, 58, 18025-18031.

(102) Yan, D.; Tang, Y.; Lin, H.; Wang, D. Tunable two-color luminescence and host-guest energy transfer of fluorescent chromophores encapsulated in metal-organic frameworks. Sci. Rep. 2014, 4, 4337.

(103) Wei, Y.; Dong, H.; Wei, C.; Zhang, W.; Yan, Y.; Zhao, Y. S. Wavelength-tunable microlasers based on the encapsulation of organic dye in metal-organic frameworks. Adv. Mater. 2016, 28, 7424-7429.

(104) Li, H.; Li, Q.; Xu, Z. Lanthanide cation encapsulated in a metal-organic framework as a white LED and selective naked-eye reversible HCl sensor. J. Mater. Chem. B 2019, 7, 2880-2885.

(105) Gao, X.; Liu, J.; Zhuang, X.; Tian, C.; Luan, F.; Liu, H.; Xiong, Y. Incorporating copper nanoclusters into a zeolitic imidazole framework-90 for use as a highly sensitive adenosine triphosphate sensing system to evaluate the freshness of aquatic products. Sens. Actuator B-Chem. 2020, 308, 127720.

(106) Chen, X.; Gao, H.; Yang, M.; Xing, L.; Dong, W.; Li, A.; Zheng, H.; Wang, G. Smart integration of carbon quantum dots in metal-organic frameworks for fluorescencefunctionalized phase change materials. Energy Storage Mater. 2019, 18, 349-355.

(107) Jena, H. S.; Kaczmarek, A. M.; Krishnaraj, C.; Feng, X.; Vijayvergia, K.; Yildirim, H.; Zhao, S.-N.; Van Deun, R.; Der Voort, P. V. White light emission properties of defect engineered metal-organic frameworks by encapsulation of $\mathrm{Eu}^{3+}$ and $\mathrm{Tb}^{3+}$. Cryst. Growth Des. 2019, 19, 6339-6350.

(108) Zhang, Y.; Li, B.; Ma, H.; Zhang, L.; Zheng, Y. Rapid and facile ratiometric detection of an anthrax biomarker by regulating energy transfer process in bio-metal-organic framework. Biosens. Bioelectron. 2016, 85, 287-293.

(109) Shen, X.; Yan, B. Barcoded materials based on photoluminescent hybrid system of lanthanide ions-doped metal organic framework and silica via ion exchange. J. Colloid Interface Sci. 2016, 468, 220-226.

(110) Zhao, X.; Wang, Y.; Hao, X.; Liu, W. Fluorescent molecule incorporated metal-organic framework for fluoride sensing in aqueous solution. Appl. Surf. Sci. 2017, 402, 129 135.

(111) Ma, Y.; Xu, G.; Wei, F.; Cen, Y.; Ma, Y.; Song, Y.; Xu, X.; Shi, M.; Muhammad, S.; $\mathrm{Hu}, \mathrm{Q}$. A dual-emissive fluorescent sensor fabricated by encapsulating quantum dots and carbon dots into metal-organic frameworks for the ratiometric detection of $\mathrm{Cu}^{2+}$ in tap water. J. Mater. Chem. B 2017, 5, 8566-8571.

(112) Othong, J.; Boonmak, J.; Promarak, V.; Kielar, F.; Youngme, S. Sonochemical synthesis of carbon dots/lanthanoid MOFs hybrids for white light-emitting diodes with high color rendering. ACS Appl. Mater. Interfaces 2019, 11, 44421-44429.

(113) Gao, Y.; Wu, J.; Wang, J.; Fan, Y.; Zhang, S.; Dai, W. A novel multifunctional p-type semiconductor@MOFs nanoporous platform for simultaneous sensing and photodegradation of tetracycline. ACS Appl. Mater. Interfaces 2020, 12, 11036-11044.

(114) Ren, J.; Zhou, X.; Wang, Y. Dual-emitting CsPbX 3 @ZJU-28 (X = Cl, Br, I) composites with enhanced stability and unique optical properties for multifunctional applications. Chem. Eng. J. 2020, 391, 123622. 
(115) Mondal, T.; Haldar, D.; Ghosh, A.; Ghorai, U. K.; Saha, S. K. A MOF functionalized with cdte quantum dots as an efficient white light emitting phosphor material for applications in displays. New J. Chem. 2020, 44, 55-63.

(116) Lyu, F.; Zhang, Y.; Zare, R. N.; Ge, J.; Liu, Z. One-pot synthesis of protein-embedded metal-organic frameworks with enhanced biological activities. Nano Lett. 2014, 14, 5761-5765.

(117) Liu, C.; Liu, Q.; Huang, A. A superhydrophobic zeolitic imidazolate framework (ZIF90) with high steam stability for efficient recovery of bioalcohols. Chem. Commun. 2016, 52, 3400-3402.

(118) Tan, H.; Wu, X.; Weng, Y.; Lu, Y.; Huang, Z.-Z. Self-assembled fret nanoprobe with metal-organic framework as a scaffold for ratiometric detection of hypochlorous acid. Anal. Chem. 2020, 92, 3447-3454.

(119) Chen, W.; Liu, H.; Fan, R.; Wang, P.; Sun, T.; Yang, Y. Formation and encapsulation of lead halide perovskites in lanthanide metal-organic frameworks for tunable emission. ACS Appl. Mater. Interfaces 2020, 12, 9851-9857.

(120) Zhang, D.; Zhou, W.; Liu, Q.; Xia, Z. $\mathrm{CH}_{3} \mathrm{NH}_{3} \mathrm{PbBr}_{3}$ perovskite nanocrystals encapsulated in lanthanide metal-organic frameworks as a photoluminescence converter for anti-counterfeiting. ACS Appl. Mater. Interfaces 2018, 10, 27875-27884.

(121) Liu, J.; Zhao, Y.; Li, X.; Wu, J.; Han, Y.; Zhang, X.; Xu, Y. Dual-emissive $\mathrm{CsPbBr}_{3} @$ Eu-BTC composite for self-calibrating temperature sensing application. Cryst. Growth Des. 2020, 20, 454-459.

(122) Mei, S.; Yang, B.; Wei, X.; Dai, H.; Chen, Z.; Cui, Z.; Zhang, G.; Xie, F.; Zhang, W.; Guo, R. Facile synthesis and optical properties of $\mathrm{CsPbX}_{3} / \mathrm{ZIF}-8$ composites for widecolor-gamut display. Nanomater. 2019, 9, 832.

(123) Lin, R.; Hou, J.; Li, M.; Wang, Z.; Ge, L.; Li, S.; Smart, S.; Zhu, Z.; Bennett, T. D.; Chen, V. Interfacial engineering of a polymer-MOF composite by in situ vitrification. Chem. Commun. 2020, 56, 3609-3612.

(124) Vu, M.-T.; Lin, R.; Diao, H.; Zhu, Z.; Bhatia, S. K.; Smart, S. Effect of ionic liquids (ILs) on MOFs/polymer interfacial enhancement in mixed matrix membranes. $J$. Membr. Sci. 2019, 587, 117157.

(125) Vornholt, S. M.; Duncan, M. J.; Warrender, S. J.; Semino, R.; Ramsahye, N. A.; Maurin, G.; Smith, M. W.; Tan, J. C.; Miller, D. N.; Morris, R. E. Multifaceted study of the interactions between $\mathrm{CPO}-27-\mathrm{Ni}$ and polyurethane and their impact on nitric oxide release performance. ACS Appl. Mater. Interfaces 2020, 12, 58263-58276.

(126) Rodenas, T.; Luz, I.; Prieto, G.; Seoane, B.; Miro, H.; Corma, A.; Kapteijn, F.; Llabres i Xamena, F. X.; Gascon, J. Metal-organic framework nanosheets in polymer composite materials for gas separation. Nat. Mater. 2015, 14, 48-55.

(127) Mehta, J. P.; Tian, T.; Zeng, Z. X.; Divitini, G.; Connolly, B. M.; Midgley, P. A.; Tan, J. C.; Fairen-Jimenez, D.; Wheatley, A. E. H. Sol-gel synthesis of robust metal-organic frameworks for nanoparticle encapsulation. Adv. Funct. Mater. 2018, 28, 1705588.

(128) Sezen, M.; Plank, H.; Nellen, P. M.; Meier, S.; Chernev, B.; Grogger, W.; Fisslthaler, E.; List, E. J.; Scherf, U.; Poelt, P. Ion beam degradation analysis of poly(3hexylthiophene) (P3HT): Can cryo-fib minimize irradiation damage? Phys. Chem. Chem. Phys. 2009, 11, 5130-5133.

(129) Fan, L.; Wang, Y.; Li, L.; Zhou, J. Carbon quantum dots activated metal organic frameworks for selective detection of $\mathrm{Cu}(\mathrm{ii})$ and Fe(iii). Colloids Surf. A 2020, 588, 124378.

(130) Fu, X.; Lv, R.; Su, J.; Li, H.; Yang, B.; Gu, W.; Liu, X. A dual-emission nano-rod MOF equipped with carbon dots for visual detection of doxycycline and sensitive sensing of $\mathrm{MnO}^{4-}$. RSC Adv. 2018, 8, 4766-4772. 
(131) Saha, S.; Das, G.; Thote, J.; Banerjee, R. Photocatalytic metal-organic framework from cds quantum dot incubated luminescent metallohydrogel. J. Am. Chem. Soc. 2014, 136, 14845-14851.

(132) Wang, Z.; Chen, R.; Xiong, Y.; Cepe, K.; Schneider, J.; Zboril, R.; Lee, C.-S.; Rogach, A. L. Incorporating copper nanoclusters into metal-organic frameworks: Confinementassisted emission enhancement and application for trinitrotoluene detection. Part. Part. Syst. Charact. 2017, 34, 1700029.

(133) Liu, L.; Zhang, D.; Zhu, Y.; Han, Y. Bulk and local structures of metal-organic frameworks unravelled by high-resolution electron microscopy. Commun. Chem. 2020, $3,99$.

(134) Li, Y.; Wang, K.; Zhou, W.; Li, Y.; Vila, R.; Huang, W.; Wang, H.; Chen, G.; Wu, G. H.; Tsao, Y.; et al. Cryo-em structures of atomic surfaces and host-guest chemistry in metal-organic frameworks. Matter 2019, 1, 428-438.

(135) Cha, J. H.; Noh, K.; Yin, W.; Lee, Y.; Park, Y.; Ahn, T. K.; Mayoral, A.; Kim, J.; Jung, D. Y.; Terasaki, O. Formation and encapsulation of all-inorganic lead halide perovskites at room temperature in metal-organic frameworks. J. Phys. Chem. Lett. 2019, 10, 2270-2277.

(136) Wang, Z.; Zhu, C.-Y.; Mo, J.-T.; Fu, P.-Y.; Zhao, Y.-W.; Yin, S.-Y.; Jiang, J.-J.; Pan, M.; Su, C.-Y. White-light emission from dual-way photon energy conversion in a dyeencapsulated metal-organic framework. Angew. Chem. Int. Ed. 2019, 58, 9752-9757.

(137) Wu, H.; Li, M.; Sun, C.; Wang, X.; Su, Z. Luminescent metal-organic frameworks encapsulating polycyclic aromatic hydrocarbons for energy transfer. Dalton Trans. 2020, 49, 5087-5091.

(138) Zhang, Z.; Wei, Z.; Meng, F.; Su, J.; Chen, D.; Guo, Z.; Xing, H. Rhb-embedded zirconium-naphthalene-based metal-organic framework composite as a luminescent self-calibrating platform for the selective detection of inorganic ions. Chem. Eur. J. 2020, 26, 1661-1667.

(139) Wei, Z.; Chen, D.; Guo, Z.; Jia, P.; Xing, H. Eosin Y-embedded zirconium-based metal-organic framework as a dual-emitting built-in self-calibrating platform for pesticide detection. Inorg. Chem. 2020, 59, 5386-5393.

(140) Liu, W.; Chen, C.; Huang, X.; Xie, E.; Liu, W. Functional construction of dual-emitting 4-aminonaphthalimide encapsulated lanthanide MOFs composite for ratiometric temperature sensing. Chem. Eur. J. 2019, 25, 10054-10058.

(141) Bagheri, M.; Masoomi, M. Y.; Morsali, A. Highly sensitive and selective ratiometric fluorescent metal-organic framework sensor to nitroaniline in presence of nitroaromatic compounds and VOCs. Sens. Actuator B-Chem. 2017, 243, 353-360.

(142) Liu, J.; Zhuang, Y.; Wang, L.; Zhou, T.; Hirosaki, N.; Xie, R.-J. Achieving multicolor long-lived luminescence in dye-encapsulated metal-organic frameworks and its application to anticounterfeiting stamps. ACS Appl. Mater. Interfaces 2018, 10, 18021809.

(143) Peterson, D. A. of Confocal microscopy. In Encyclopedia of movement disorders; Kompoliti, K.;Metman, L. V., Eds.; Academic Press: Oxford, 2010; pp 250-252.

(144) Caballero-Mancebo, E.; Cohen, B.; Moreno, J. M.; Corma, A.; Díaz, U.; Douhal, A. Exploring the photodynamics of a new 2D-MOF composite: Nile red@Al-ITQ-HB. ACS Omega 2018, 3, 1600-1608.

(145) Dong, J.; Qiao, Z.; Pan, Y.; Peh, S. B.; Yuan, Y. D.; Wang, Y.; Zhai, L.; Yuan, H.; Cheng, Y.; Liang, H.; et al. Encapsulation and protection of ultrathin two-dimensional porous organic nanosheets within biocompatible metal-organic frameworks for livecell imaging. Chem. Mater. 2019, 31, 4897-4912. 
(146) Blight, B. A.; Ahmad, T. I.; Shepherd, H. J.; Jennings, C. S.; Ferland, L. I.; Teat, S. J.; Rossman, J. S. Sterol uptake by an alkali- $\beta$-cyclodextrin metal-organic framework. Cryst. Growth Des. 2019, 20, 43-48.

(147) Choi, I. H.; Bin Yoon, S.; Huh, S.; Kim, S. J.; Kim, Y. Photophysical properties of cationic dyes captured in the mesoscale channels of micron-sized metal-organic framework crystals. Sci. Rep. 2018, 8, 9838.

(148) Möslein, A. F.; Gutiérrez, M.; Cohen, B.; Tan, J.-C. Near-field infrared nanospectroscopy reveals guest confinement in metal-organic framework single crystals. Nano Lett. 2020, 20, 7446-7454.

(149) Keilmann, F.; Hillenbrand, R. Near-field microscopy by elastic light scattering from a tip. Phil. Trans. R. Soc. Lond. A 2004, 362, 787-805.

(150) Penner-Hahn, J. E. X-ray absorption spectroscopy. http://websites.umich.edu/ jphgroup/XAS_Course/Harbin/CCC2_XAS.pdf (accessed 2021-11-14).

(151) Bordiga, S.; Bonino, F.; Lillerud, K. P.; Lamberti, C. X-ray absorption spectroscopies: Useful tools to understand metallorganic frameworks structure and reactivity. Chem. Soc. Rev. 2010, 39, 4885-4927.

(152) Mustafa, D.; Silva, I. G.; Bajpe, S. R.; Martens, J. A.; Kirschhock, C. E.; Breynaert, E.; Brito, H. F.Eu@COK-16, a host sensitized, hybrid luminescent metal-organic framework. Dalton Trans. 2014, 43, 13480-13484.

(153) Titov, K.; Eremin, D. B.; Kashin, A. S.; Boada, R.; Souza, B. E.; Kelley, C. S.; Frogley, M. D.; Cinque, G.; Gianolio, D.; Cibin, G.; et al. OX-1 metal-organic framework nanosheets as robust hosts for highly active catalytic palladium species. ACS Sustain. Chem. Eng. 2019, 7, 5875-5885.

(154) Ryder, M. R.; Civalleri, B.; Bennett, T. D.; Henke, S.; Rudić, S.; Cinque, G.; Fernandez-Alonso, F.; Tan, J. C. Identifying the role of terahertz vibrations in metalorganic frameworks: From gate-opening phenomenon to shear-driven structural destabilization. Phys. Rev. Lett. 2014, 113, 215502.

(155) Kuchta, B.; Formalik, F.; Rogacka, J.; Neimark, A. V.; Firlej, L. Phonons in deformable microporous crystalline solids. Z. Kristall. Cryst. Mater. 2019, 234, 513-527.

(156) Hoffman, A. E. J.; Wieme, J.; Rogge, S. M. J.; Vanduyfhuys, L.; Van Speybroeck, V. The impact of lattice vibrations on the macroscopic breathing behavior of MIL-53(al). Z. Kristall. Cryst. Mater. 2019, 234, 529-545.

(157) Ryder, M. R.; Civalleri, B.; Cinque, G.; Tan, J. C. Discovering connections between terahertz vibrations and elasticity underpinning the collective dynamics of the HKUST1 metal-organic framework. CrystEngComm 2016, 18, 4303-4312.

(158) Kieslich, G.; Skelton, J. M.; Armstrong, J.; Wu, Y.; Wei, F.; Svane, K. L.; Walsh, A.; Butler, K. T. Hydrogen bonding versus entropy: Revealing the underlying thermodynamics of the hybrid organic-inorganic perovskite $\left[\mathrm{ch}_{3} \mathrm{nh}_{3}\right] \mathrm{pbbr}_{3}$. Chem. Mater. 2018, 30, 8782-8788.

(159) Ryder, M. R.; Van de Voorde, B.; Civalleri, B.; Bennett, T. D.; Mukhopadhyay, S.; Cinque, G.; Fernandez-Alonso, F.; De Vos, D.; Rudic, S.; Tan, J. C. Detecting molecular rotational dynamics complementing the low-frequency terahertz vibrations in a zirconium-based metal-organic framework. Phys. Rev. Lett. 2017, 118, 255502.

(160) Neutron scattering - fundamentals, experimental methods in the physical sciences; Fernandez-Alonso, F.;Price, D. L., Eds.; Academic Press: New York, 2013; pp 39-41.

(161) Tan, N. Y.; Ruggiero, M. T.; Orellana-Tavra, C.; Tian, T.; Bond, A. D.; Korter, T. M.; Fairen-Jimenez, D.; Zeitler, J. A. Investigation of the terahertz vibrational modes of ZIF-8 and ZIF-90 with terahertz time-domain spectroscopy. Chem. Commun. 2015, 51, 16037-16040. 
(162) Cavaye, H.; Schastny, M. In situ illumination with inelastic neutron scattering: A study of the photochromic material cis-1,2-dicyano-1,2-bis(2,4,5-trimethyl-3-thienyl)ethene (CMTE). Phys. Chem. Chem. Phys. 2021, 23, 22324-22329.

(163) Ryder, M. R.; Bennett, T. D.; Kelley, C. S.; Frogley, M. D.; Cinque, G.; Tan, J. C. Tracking thermal-induced amorphization of a zeolitic imidazolate framework via synchrotron in situ far-infrared spectroscopy. Chem. Commun. 2017, 53, 7041-7044.

(164) Hobday, C. L.; Woodall, C. H.; Lennox, M. J.; Frost, M.; Kamenev, K.; Duren, T.; Morrison, C. A.; Moggach, S. A. Understanding the adsorption process in ZIF-8 using high pressure crystallography and computational modelling. Nat. Commun. 2018, 9, 1429.

(165) Cuadrado-Collados, C.; Fernandez-Catala, J.; Fauth, F.; Cheng, Y. Q. Q.; Daemen, L. L.; Ramirez-Cuesta, A. J.; Silvestre-Albero, J. Understanding the breathing phenomena in nano-ZIF-7 upon gas adsorption. J. Mater. Chem. A 2017, 5, 20938-20946.

(166) Zhang, X.; da Silva, I.; Godfrey, H. G. W.; Callear, S. K.; Sapchenko, S. A.; Cheng, Y.; Vitórica-Yrezábal, I.; Frogley, M. D.; Cinque, G.; Tang, C. C.; et al. Confinement of iodine molecules into triple-helical chains within robust metal-organic frameworks. J. Am. Chem. Soc. 2017, 139, 16289-16296.

(167) Souza, B. E.; Dona, L.; Titov, K.; Bruzzese, P.; Zeng, Z.; Zhang, Y.; Babal, A. S.; Moslein, A. F.; Frogley, M. D.; Wolna, M.; et al. Elucidating the drug release from metal-organic framework nanocomposites via in situ synchrotron microspectroscopy and theoretical modeling. ACS Appl. Mater. Interfaces 2020, 12, 5147-5156.

(168) Zhao, P.; Fang, H.; Mukhopadhyay, S.; Li, A.; Rudić, S.; McPherson, I. J.; Tang, C. C.; Fairen-Jimenez, D.; Tsang, S. C. E.; Redfern, S. A. T. Structural dynamics of a metalorganic framework induced by $\mathrm{CO}_{2}$ migration in its non-uniform porous structure. Nat. Commun. 2019, 10, 999.

(169) Souza, B. E.; Rudic, S.; Titov, K.; Babal, A. S.; Taylor, J. D.; Tan, J. C. Guest-host interactions of nanoconfined anti-cancer drug in metal-organic framework exposed by terahertz dynamics. Chem. Commun. 2019, 55, 3868-3871.

(170) Easun, T. L.; Moreau, F.; Yan, Y.; Yang, S. H.; Schroder, M. Structural and dynamic studies of substrate binding in porous metal-organic frameworks. Chem. Soc. Rev. 2017, 46, 239-274.

(171) Mayorga-Gonzalez, R.; Rivera-Torrente, M.; Nikolopoulos, N.; Bossers, K. W.; Valadian, R.; Yus, J.; Seoane, B.; Weckhuysen, B. M.; Meirer, F. Visualizing defects and pore connectivity within metal-organic frameworks by $\mathrm{X}$-ray transmission tomography. Chem. Sci. 2021, 12, 8458-8467.

(172) Arul, K. T.; Chang, H.-W.; Shiu, H.-W.; Dong, C.-L.; Pong, W.-F. A review of energy materials studied by in situ/operando synchrotron X-ray spectro-microscopy. J. Phys. D-Appl. Phys. 2021, 54, 343001.

(173) Ferreira Sanchez, D.; Ihli, J.; Zhang, D.; Rohrbach, T.; Zimmermann, P.; Lee, J.; Borca, C. N.; Bohlen, N.; Grolimund, D.; van Bokhoven, J. A.; et al. Spatio-chemical heterogeneity of defect-engineered metal-organic framework crystals revealed by fullfield tomographic X-ray absorption spectroscopy. Angew. Chem. Int. Ed. 2021, 60, 10032-10039.

(174) Preat, J. Photoinduced energy-transfer and electron-transfer processes in dye-sensitized solar cells: TDDFT insights for triphenylamine dyes. J. Phys. Chem. C 2010, 114, 16716-16725.

(175) Marchioro, A.; Teuscher, J.; Friedrich, D.; Kunst, M.; van de Krol, R.; Moehl, T.; Grätzel, M.; Moser, J.-E. Unravelling the mechanism of photoinduced charge transfer processes in lead iodide perovskite solar cells. Nat. Photonics 2014, 8, 250-255. 
(176) Liu, T.; Chen, X.; Zhao, J.; Wei, W.; Mao, Z.; Wu, W.; Jiao, S.; Liu, Y.; Yang, Z.; Chi, Z. Hybridized local and charge-transfer excited state fluorophores enabling organic light-emitting diodes with record high efficiencies close to $20 \%$. Chem. Sci. 2021, 12, 5171-5176.

(177) Park, H.; Kim, H.-i.; Moon, G.-h.; Choi, W. Photoinduced charge transfer processes in solar photocatalysis based on modified $\mathrm{TiO}_{2}$. Energy Environ. Sci. 2016, 9, 411-433.

(178) di Nunzio, M. R.; Gutiérrez, M.; Douhal, A. of Chapter 15 - electronic and molecular motions in silica-material hosts. In Chemistry of silica and zeolite-based materials; Douhal, A.;Anpo, M., Eds.; Elsevier, 2019; vol. 2; pp 273-294.

(179) Moissette, A.; Hureau, M.; Vezin, H.; Lobo, R. F. of Chapter 14 - electron transfers under confinement in channel-type zeolites. In Chemistry of silica and zeolite-based materials; Douhal, A.;Anpo, M., Eds.; Elsevier, 2019; vol. 2; pp 249-271.

(180) di Nunzio, M. R.; Caballero-Mancebo, E.; Cohen, B.; Douhal, A. Photodynamical behaviour of MOFs and related composites: Relevance to emerging photon-based science and applications. J. Photochem. Photobiol. C 2020, 44, 100355.

(181) So, M. C.; Wiederrecht, G. P.; Mondloch, J. E.; Hupp, J. T.; Farha, O. K. Metal-organic framework materials for light-harvesting and energy transfer. Chem. Commun. 2015, 51,3501-3510.

(182) Cao, W.; Tang, Y.; Cui, Y.; Qian, G. Energy transfer in metal-organic frameworks and its applications. Small Struct. 2020, 1, 2000019.

(183) Mu, Q.; Liu, J.; Chen, W.; Song, X.; Liu, X.; Zhang, X.; Chang, Z.; Chen, L. A new biscarbazole-based metal-organic framework for efficient host-guest energy transfer. Chem. Eur. J. 2019, 25, 1901-1905.

(184) Seo, C.; Kim, M.; Lee, J.; Lee, C. Y.; Kim, J. Spectroscopic evidence of energy transfer in bodipy-incorporated nano-porphyrinic metal-organic frameworks. Nanomater. 2020, 10, 1925.

(185) Krause, G. H.; Weis, E. Chlorophyll fluorescence and photosynthesis: The basics. Annu. Rev. Plant Physiol. Plant Mol. Biol. 1991, 42, 313-349.

(186) Goes, M.; Verhoeven, J. W.; Hofstraat, H.; Brunner, K. Oled and pled devices employing electrogenerated, intramolecular charge-transfer fluorescence. ChemPhysChem 2003, 4, 349-358.

(187) Tang, X.; Cui, L. S.; Li, H. C.; Gillett, A. J.; Auras, F.; Qu, Y. K.; Zhong, C.; Jones, S. T. E.; Jiang, Z. Q.; Friend, R. H.; et al. Highly efficient luminescence from spaceconfined charge-transfer emitters. Nat. Mater. 2020, 19, 1332-1338.

(188) Li, X.; Surendran Rajasree, S.; Yu, J.; Deria, P. The role of photoinduced charge transfer for photocatalysis, photoelectrocatalysis and luminescence sensing in metalorganic frameworks. Dalton Trans. 2020, 49, 12892-12917.

(189) Leong, K.; Foster, M. E.; Wong, B. M.; Spoerke, E. D.; Van Gough, D.; Deaton, J. C.; Allendorf, M. D. Energy and charge transfer by donor-acceptor pairs confined in a metal-organic framework: A spectroscopic and computational investigation. J. Mater. Chem. A 2014, 2, 3389-3398.

(190) Caballero-Mancebo, E.; Moreno, J. M.; Cohen, B.; Díaz, U.; Corma, A.; Douhal, A. Unraveling competitive electron and energy-transfer events at the interfaces of a $2 \mathrm{D}$ MOF and nile red composites: Effect of the length and structure of the linker. ACS Appl. Mater. Interfaces 2018, 10, 32885-32894.

(191) Caballero-Mancebo, E.; Moreno, J. M.; Corma, A.; Díaz, U.; Cohen, B.; Douhal, A. How does the surface of Al-ITQ-HB 2D-MOF condition the intermolecular interactions of an adsorbed organic molecule? ACS Appl. Mater. Interfaces 2018, 10, 20159-20169. 
(192) Li, X.; Yang, L.; Zhao, L.; Wang, X.-L.; Shao, K.-Z.; Su, Z.-M. Luminescent metalorganic frameworks with anthracene chromophores: Small-molecule sensing and highly selective sensing for nitro explosives. Cryst. Growth Des. 2016, 16, 4374-4382.

(193) Tian, D.; Li, Y.; Chen, R.-Y.; Chang, Z.; Wang, G.-Y.; Bu, X.-H. A luminescent metalorganic framework demonstrating ideal detection ability for nitroaromatic explosives. J. Mater. Chem. A 2014, 2, 1465-1470.

(194) Nagarkar, S. S.; Joarder, B.; Chaudhari, A. K.; Mukherjee, S.; Ghosh, S. K. Highly selective detection of nitro explosives by a luminescent metal-organic framework. 2013, 52, 2881-2885.

(195) Gutiérrez, M.; Navarro, R.; Sánchez, F.; Douhal, A. Photodynamics of Zr-based MOFs: Effect of explosive nitroaromatics. Phys. Chem. Chem. Phys. 2017, 19, 16337-16347.

(196) Arnaut, L. G.; Formosinho, S. J. Excited-state proton transfer reactions i. Fundamentals and intermolecular reactions. J. Photochem. Photobiol. A: Chem. 1993, 75, 1-20.

(197) Weller, A. Quantitative untersuchungen der fluoreszenzumwandlung bei naphtholen. Z. Elektrochem. 1952, 56, 662-668.

(198) Weller, A. Outer and inner mechanism of reactions of excited molecules. Discuss. Faraday Soc. 1959, 27, 28-33.

(199) Weber, K. Close connection between extinction of fluorescence and retardation of photochemical reactions. Z. Physik. Chem 1931, 15, 18-44.

(200) Förster, T. Fluoreszenzspektrum und wasserstoffionen-konzentration. Naturwissenschaften 1949, 36, 186-187.

(201) Lei, J.; Wang, B.; Li, Y.-P.; Ji, W.-J.; Wang, K.; Qi, H.; Chou, P.-T.; Zhang, M.-M.; Bian, H.; Zhai, Q.-G. A new molecular recognition concept: Multiple hydrogen bonds and their optically triggered proton transfer in confined metal-organic frameworks for superior sensing element. ACS Appl. Mater. Interfaces 2021, 13, 22457-22465.

(202) Halder, A.; Bhattacharya, B.; Haque, F.; Dinda, S.; Ghoshal, D. Polarity-induced excited-state intramolecular proton transfer (ESIPT) in a pair of supramolecular isomeric multifunctional dynamic metal-organic frameworks. Chem. Eur. J. 2019, 25, 12196-12205.

(203) Li, Y.-P.; Zhu, X.-H.; Li, S.-N.; Jiang, Y.-C.; Hu, M.-C.; Zhai, Q.-G. Highly selective and sensitive turn-off-on fluorescent probes for sensing $\mathrm{Al}^{3+}$ ions designed by regulating the excited-state intramolecular proton transfer process in metal-organic frameworks. ACS Appl. Mater. Interfaces 2019, 11, 11338-11348.

(204) Yan, X.; Li, Y.-P.; Lei, J.; Wang, Y.; Li, S.-N.; Zhai, Q.-G. Introduction of continuous excited-state intermolecular proton transfer process into open yttrium-terephthalate framework for ratiometric fluorescent fluorion detection. J. Solid State Chem. 2021, $300,122212$.

(205) Jayaramulu, K.; Kanoo, P.; George, S. J.; Maji, T. K. Tunable emission from a porous metal-organic framework by employing an excited-state intramolecular proton transfer responsive ligand. Chem. Commun. 2010, 46, 7906-7908.

(206) Chen, L.; Ye, J.-W.; Wang, H.-P.; Pan, M.; Yin, S.-Y.; Wei, Z.-W.; Zhang, L.-Y.; Wu, K.; Fan, Y.-N.; Su, C.-Y. Ultrafast water sensing and thermal imaging by a metalorganic framework with switchable luminescence. Nat. Commun. 2017, 8, 15985.

(207) Huang, P.; Liu, Y.; Karmakar, A.; Yang, Q.; Li, J.; Wu, F.-Y.; Deng, K.-Y. Tuning the excited-state intramolecular proton transfer (ESIPT)-based luminescence of metalorganic frameworks by metal nodes toward versatile photoluminescent applications. Dalton Trans. 2021, 50, 6901-6912.

(208) Newsome, W. J.; Ayad, S.; Cordova, J.; Reinheimer, E. W.; Campiglia, A. D.; Harper, J. K.; Hanson, K.; Uribe-Romo, F. J. Solid state multicolor emission in substitutional 
solid solutions of metal-organic frameworks. J. Am. Chem. Soc. 2019, 141, 1129811303.

(209) Othong, J.; Boonmak, J.; Wannarit, N.; Kielar, F.; Puangmali, T.; Phanchai, W.; Youngme, S. Dual mode in a metal-organic framework based mixed matrix membrane for discriminative detection of amines: Vapoluminescent and vapochromic response. Sens. Actuator B-Chem. 2021, 343, 130066.

(210) Duarte, F. J. of Chapter 5 - dye lasers. In Tunable Lasers Handbook; Duarte, F. J., Ed.; Academic Press: San Diego, 1995; pp 167-218.

(211) Glembockyte, V.; Frenette, M.; Mottillo, C.; Durantini, A. M.; Gostick, J.; Štrukil, V.; Friščić, T.; Cosa, G. Highly photostable and fluorescent microporous solids prepared via solid-state entrapment of boron dipyrromethene dyes in a nascent metal-organic framework. J. Am. Chem. Soc. 2018, 140, 16882-16887.

(212) McKeithan, C. R.; Mayers, J. M.; Wojtas, L.; Larsen, R. W. Photophysical studies of $\mathrm{Ru}(\mathrm{II})$ tris(2,2'-bipyridine) encapsulated within the ZnHKUST-1 metal organic framework. Inorg. Chim. Acta 2018, 483, 1-5.

(213) Mayers, J. M.; Larsen, R. W. Photophysical study of $\left[\mathrm{Ru}\left(2,2^{\prime} \text {-bipyridine }\right)_{3}\right]^{2+}$ and $\left[\mathrm{Ru}(1,10 \text {-phenanthroline })_{3}\right]^{2+}$ encapsulated in the UiO-66- $\mathrm{NH}_{2}$ metal organic framework. Polyhedron 2019, 171, 382-388.

(214) Larsen, R. W.; Wojtas, L. Photophysical properties of $\left[\mathrm{Ru}\left(2,2^{\prime} \text {-bipyridine }\right)_{3}\right]^{2+}$ encapsulated within the UiO-66 zirconium based metal organic framework. J. Solid State Chem. 2017, 247, 77-82.

(215) Li, Y.; Wei, Z.; Zhang, Y.; Guo, Z.; Chen, D.; Jia, P.; Chen, P.; Xing, H. Dual-emitting EY@Zr-MOF composite as self-calibrating luminescent sensor for selective detection of inorganic ions and nitroaromatics. ACS Sustain. Chem. Eng. 2019, 7, 6196-6203.

(216) Kasha, M.; Rawls, H. R.; Ashraf El-Bayoumi, M. The exciton model in molecular spectroscopy. Pure Appl. Chem. 1965, 11, 371-392.

(217) McRae, E. G.; Kasha, M. Enhancement of phosphorescence ability upon aggregation of dye molecules. J. Chem. Phys. 1958, 28, 721-722.

(218) Yi, F.-Y.; Chen, D.; Wu, M.-K.; Han, L.; Jiang, H.-L. Chemical sensors based on metalorganic frameworks. ChemPlusChem 2016, 81, 675-690.

(219) Kreno, L. E.; Leong, K.; Farha, O. K.; Allendorf, M.; Van Duyne, R. P.; Hupp, J. T. Metal-organic framework materials as chemical sensors. Chem. Rev. 2012, 112, $1105-$ 1125.

(220) Karmakar, A.; Samanta, P.; Dutta, S.; Ghosh, S. K. Fluorescent "turn-on" sensing based on metal-organic frameworks (MOFs). Chem. Asian. J. 2019, 14, 4506-4519.

(221) Gao, M.; Tang, B. Z. Fluorescent sensors based on aggregation-induced emission: Recent advances and perspectives. ACS Sens. 2017, 2, 1382-1399.

(222) Chen, D. M.; Zhang, N. N.; Liu, C. S.; Du, M. Dual-emitting dye@MOF composite as a self-calibrating sensor for 2,4,6-trinitrophenol. ACS Appl. Mater. Interfaces 2017, 9, 24671-24677.

(223) Yin, H. Q.; Yang, J. C.; Yin, X. B. Ratiometric fluorescence sensing and real-time detection of water in organic solvents with one-pot synthesis of Ru@MIL-101(al)-NH2. Anal. Chem. 2017, 89, 13434-13440.

(224) Zhang, X.; Hu, Q.; Xia, T.; Zhang, J.; Yang, Y.; Cui, Y.; Chen, B.; Qian, G. Turn-on and ratiometric luminescent sensing of hydrogen sulfide based on metal-organic frameworks. ACS Appl. Mater. Interfaces 2016, 8, 32259-32265.

(225) Gutiérrez, M.; Martín, C.; Souza, B. E.; Van der Auweraer, M.; Hofkens, J.; Tan, J.-C. Highly luminescent silver-based MOFs: Scalable eco-friendly synthesis paving the way for photonics sensors and electroluminescent devices. Appl. Mater. Today 2020, 21, 100817. 
(226) Hinckley, D. A.; Seybold, P. G.; Borris, D. P. Solvatochromism and thermochromism of rhodamine solutions. Spectroc. Acta A: Mol. Biomol. Spectrosc. 1986, 42, 747-754.

(227) Rosenthal, I.; Peretz, P.; Muszkat, K. A. Thermochromic and hyperchromic effects in rhodamine B solutions. J. Phys. Chem. 1979, 83, 350-353.

(228) Takei, Y.; Arai, S.; Murata, A.; Takabayashi, M.; Oyama, K.; Ishiwata, S.; Takeoka, S.; Suzuko, M. A nanoparticle-based ratiometric and self-calibrated fluorescent thermometer for single living cells. ACS Nano 2014, 8, 198-206.

(229) Karstens, T.; Kobs, K. Rhodamine B and rhodamine 101 as reference substances for fluorescence quantum yield measurements. J. Phys. Chem. 1980, 84, 1871-1872.

(230) Zhou, Y.; Zhang, D.; Zeng, J.; Gan, N.; Cuan, J. A luminescent lanthanide-free MOF nanohybrid for highly sensitive ratiometric temperature sensing in physiological range. Talanta 2018, 181, 410-415.

(231) Chi, Z.; Zhang, X.; Xu, B.; Zhou, X.; Ma, C.; Zhang, Y.; Liu, S.; Xu, J. Recent advances in organic mechanofluorochromic materials. Chem. Soc. Rev. 2012, 41, 3878-3896.

(232) Roberts, D. R. T.; Holder, S. J. Mechanochromic systems for the detection of stress, strain and deformation in polymeric materials. J. Mater. Chem. 2011, 21, 8256-8268.

(233) Sun, J. K.; Chen, C.; Cai, L. X.; Ren, C. X.; Tan, B.; Zhang, J. Mechanical grinding of a single-crystalline metal-organic framework triggered emission with tunable violet-toorange luminescence. Chem. Commun. 2014, 50, 15956-15959.

(234) Wen, T.; Zhou, X. P.; Zhang, D. X.; Li, D. Luminescent mechanochromic porous coordination polymers. Chem. Eur. J. 2014, 20, 644-648.

(235) Deshmukh, M. S.; Yadav, A.; Pant, R.; Boomishankar, R. Thermochromic and mechanochromic luminescence umpolung in isostructural metal-organic frameworks based on $\mathrm{Cu}_{6} \mathrm{I}_{6}$ clusters. Inorg. Chem. 2015, 54, 1337-1345.

(236) Zhang, Q.; Su, J.; Feng, D.; Wei, Z.; Zou, X.; Zhou, H. C. Piezofluorochromic metalorganic framework: A microscissor lift. J. Am. Chem. Soc. 2015, 137, 10064-10067.

(237) Wen, T.; Zheng, Y.; Xu, C.; Zhang, J.; Jaroniec, M.; Qiao, S.-Z. A boron imidazolate framework with mechanochromic and electrocatalytic properties. Mater. Horiz. 2018, 5, 1151-1155.

(238) Xu, B.; Qi, Q.; Zhang, J.; Tian, W. of Chapter 7: Mechanofluorochromic mechanism. In Mechanochromic fluorescent materials: Phenomena, materials and applications; Xu, J.;Chi, Z., Eds.; RSC Publishing: Cambridge, 2014; pp 236-262.

(239) Wang, H.; Zhao, E.; Lam, J. W. Y.; Tang, B. Z. AIE luminogens: Emission brightened by aggregation. Mater. Today 2015, 18, 365-377.

(240) Zhang, Y.; Xiong, T.; Möslein, A. F.; Mollick, S.; Kachwal, V.; Babal, A. S.; Tan, J. C. Nanoconfinement of tetraphenylethylene in zeolitic metal-organic framework for turn-on mechanofluorochromic stress sensing. Appl. Mater. Today 2022, In Press (https://doi.org/10.1016/j.apmt.2022.101434), 101434.

(241) Jin, H.-G.; Zong, W.; Yuan, L.; Zhang, X.-B. Nanoscale zeolitic imidazole framework90: Selective, sensitive and dual-excitation ratiometric fluorescent detection of hazardous Cr(VI) anions in aqueous media. New J. Chem. 2018, 42, 12549-12556.

(242) Karmakar, A.; Joarder, B.; Mallick, A.; Samanta, P.; Desai, A. V.; Basu, S.; Ghosh, S. $\mathrm{K}$. Aqueous phase sensing of cyanide ions using a hydrolytically stable metal-organic framework. Chem. Commun. 2017, 53, 1253-1256.

(243) Zheng, H. Y.; Lian, X.; Qin, S. J.; Yan, B. Novel "turn-on" fluorescent probe for highly selectively sensing fluoride in aqueous solution based on $\mathrm{Tb}^{3+}$-functionalized metalorganic frameworks. ACS Omega 2018, 3, 12513-12519.

(244) Hao, J. N.; Yan, B. A water-stable lanthanide-functionalized MOF as a highly selective and sensitive fluorescent probe for $\mathrm{Cd}^{2+}$. ChemComm. 2015, 51, 7737-7740. 
(245) Roy, I.; Bobbala, S.; Zhou, J.; Nguyen, M. T.; Nalluri, S. K. M.; Wu, Y.; Ferris, D. P.; Scott, E. A.; Wasielewski, M. R.; Stoddart, J. F. Extzbox: A glowing cyclophane for live-cell imaging. J. Am. Chem. Soc. 2018, 140, 7206-7212.

(246) Cao, X.; Cheng, S.; You, Y.; Zhang, S.; Xian, Y. Sensitive monitoring and bioimaging intracellular highly reactive oxygen species based on gold nanoclusters@nanoscale metal-organic frameworks. Anal. Chim. Acta 2019, 1092, 108-116.

(247) Chowdhuri, A. R.; Singh, T.; Ghosh, S. K.; Sahu, S. K. Carbon dots embedded magnetic nanoparticles@chitosan@metal organic framework as a nanoprobe for pH sensitive targeted anticancer drug delivery. ACS Appl. Mater. Interfaces 2016, 8, 16573-16583.

(248) Gao, X.; Wang, Y.; Ji, G.; Cui, R.; Liu, Z. One-pot synthesis of hierarchical-pore metalorganic frameworks for drug delivery and fluorescent imaging. CrystEngComm 2018, 20, 1087-1093.

(249) Su, F.; Jia, Q.; Li, Z.; Wang, M.; He, L.; Peng, D.; Song, Y.; Zhang, Z.; Fang, S. Aptamer-templated silver nanoclusters embedded in zirconium metal-organic framework for targeted antitumor drug delivery. Microporous Mesoporous Mat. 2019, $275,152-162$.

(250) Shi, L.; Wang, J.; Zhou, L.; Chen, Y.; Yan, J.; Dai, C. Facile in-situ preparation of MAPbBr $3 @$ UiO-66 composites for information encryption and decryption. J. Solid State Chem. 2020, 282, 121062.

(251) U.S. Department energy. Lighting choices to save you money. https://www.energy.gov/energysaver/lighting-choices-save-you-money (accessed 2021-11-14).

(252) Balaram, V. Rare earth elements: A review of applications, occurrence, exploration, analysis, recycling, and environmental impact. Geosci. Front. 2019, 10, 1285-1303.

(253) Zhao, M.; Liao, H.; Molokeev, M. S.; Zhou, Y.; Zhang, Q.; Liu, Q.; Xia, Z. Emerging ultra-narrow-band cyan-emitting phosphor for white LEDs with enhanced color rendition. Light Sci. Appl. 2019, 8, 38.

(254) Wang, X.; Li, Z.; Ying, W.; Chen, D.; Li, P.; Deng, Z.; Peng, X. Blue metal-organic framework encapsulated denatured R-phycoerythrin proteins for a white-light-emitting thin film. J. Mater. Chem. B 2020, 8, 240-246.

(255) Tang, Y.; Xia, T.; Song, T.; Cui, Y.; Yang, Y.; Qian, G. Efficient energy transfer within dyes encapsulated metal-organic frameworks to achieve high performance white lightemitting diodes. Adv. Opt. Mater. 2018, 6, 1800968.

(256) Wang, A.; Hou, Y.-L.; Kang, F.; Lyu, F.; Xiong, Y.; Chen, W.-C.; Lee, C.-S.; Xu, Z.; Rogach, A. L.; Lu, J.; et al. Rare earth-free composites of carbon dots/metal-organic frameworks as white light emitting phosphors. J. Mater. Chem. B 2019, 7, 2207-2211.

(257) Gutiérrez, M.; Martin, C.; Kennes, K.; Hofkens, J.; Van der Auweraer, M.; Sánchez, F.; Douhal, A. New OLEDs based on zirconium metal-organic framework. Adv. Opt. Mater. 2018, 6, 1701060.

(258) Chen, D.; Xing, H.; Su, Z.; Wang, C. Electrical conductivity and electroluminescence of a new anthracene-based metal-organic framework with $\pi$-conjugated zigzag chains. Chem. Commun. 2016, 52, 2019-2022.

(259) Haider, G.; Usman, M.; Chen, T.-P.; Perumal, P.; Lu, K.-L.; Chen, Y.-F. Electrically driven white light emission from intrinsic metal-organic framework. ACS Nano 2016, $10,8366-8375$.

(260) Haldar, R.; Jakoby, M.; Kozlowska, M.; Rahman Khan, M.; Chen, H.; Pramudya, Y.; Richards, B. S.; Heinke, L.; Wenzel, W.; Odobel, F.; et al. Tuning optical properties by controlled aggregation: Electroluminescence assisted by thermally-activated delayed 
fluorescence from thin films of crystalline chromophores. Chem. Eur. J. 2020, 26, 17016-17020.

(261) Farahani, R. D.; Dube, M.; Therriault, D. Three-dimensional printing of multifunctional nanocomposites: Manufacturing techniques and applications. Adv. Mater. 2016, 28, 5794-5821.

(262) Hohmann, J. K.; Renner, M.; Waller, E. H.; von Freymann, G. Three-dimensional $\mu-$ printing: An enabling technology. Adv. Opt. Mater. 2015, 3, 1488-1507.

(263) Stolar, T.; Užarević, K. Mechanochemistry: An efficient and versatile toolbox for synthesis, transformation, and functionalization of porous metal-organic frameworks. CrystEngComm 2020, 22, 4511-4525.

(264) Souza, B. E.; Tan, J.-C. Mechanochemical approaches towards the in situ confinement of 5-FU anti-cancer drug within MIL-100 (Fe) metal-organic framework. CrystEngComm 2020, 22, 4526-4530.

(265) Dona, L.; Brandenburg, J. G.; Civalleri, B. Extending and assessing composite electronic structure methods to the solid state. J. Chem. Phys. 2019, 151, 121101.

(266) Lencione, D.; Gehlen, M. H.; Trujillo, L. N.; Leitao, R. C.; Albuquerque, R. Q. The spatial distribution of the photostability of thionine in zeolite 1 nanochannels investigated by photobleaching lifetime imaging microscopy. Photochem. Photobiol. Sci. 2016, 15, 398-404.

(267) Sumida, A.; Imoto, H.; Naka, K. Turn-on type sensing of methanol vapor by a luminescent platinum(II) dichloride complex with 21-dibenzoarsacrown-7. Dalton Trans. 2021, 50, 6682-6687.

(268) Zeng, Z.; Tan, J. C. AFM nanoindentation to quantify mechanical properties of nanoand micron-sized crystals of a metal-organic framework material. ACS Appl. Mater. Interfaces 2017, 9, 39839-39854.

(269) Stepniewska, M.; Januchta, K.; Zhou, C.; Qiao, A.; Smedskjaer, M. M.; Yue, Y. Observation of indentation-induced shear bands in a metal-organic framework glass. Proc. Natl. Acad. Sci. USA 2020, 117, 10149-10154.

(270) Zeng, Z.; Flyagina, I. S.; Tan, J.-C. Nanomechanical behavior and interfacial deformation beyond the elastic limit in 2D metal-organic framework nanosheets. Nanoscale Adv. 2020, 2, 5181-5191. 